\title{
THE SKELETO-MUSCULAR MECHANISMS OF THE HONEY BEE
}

\author{
By R. E. SNODGRASS
}

Bureau of Entomology and Plant Quarantine, U. S. Department of Agriculture

\section{CONTENTS}

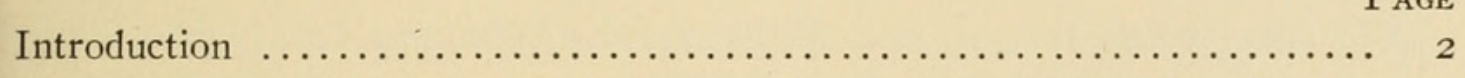

I. The head and the feeding apparatus $\ldots \ldots \ldots \ldots \ldots \ldots \ldots \ldots \ldots, 4$

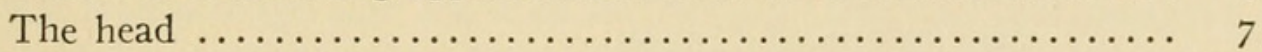

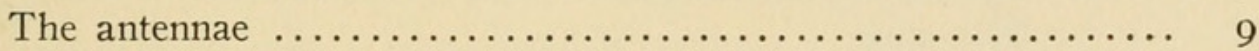

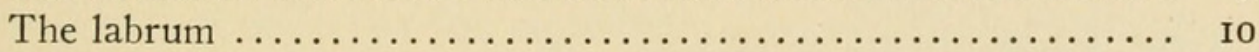

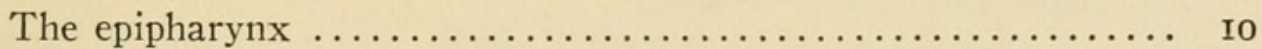

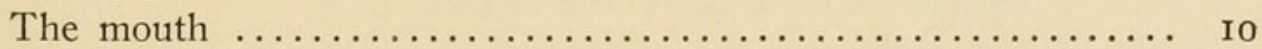

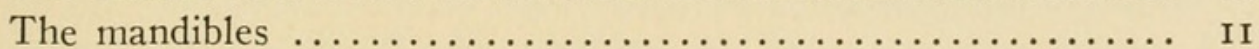

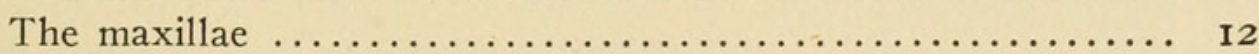

The labium and hypopharynx $\ldots \ldots \ldots \ldots \ldots \ldots \ldots \ldots \ldots \ldots \ldots \ldots \ldots \ldots$

The preoral food channel........................ 27

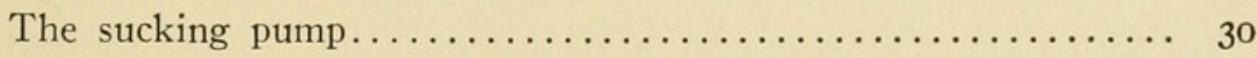

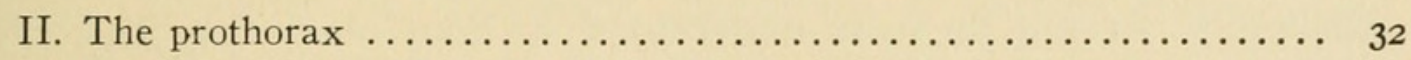

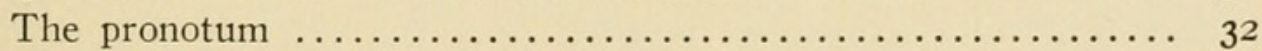

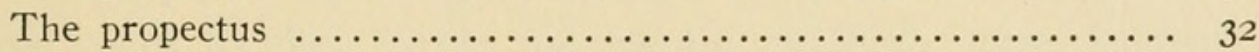

The prothoracic endosternum...................... 33

The prothoracic muscles...................... 35

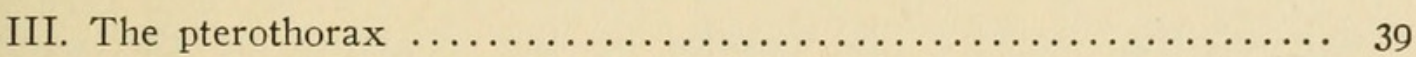

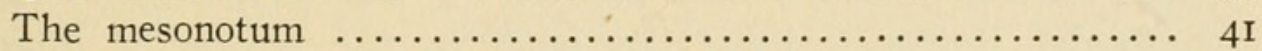

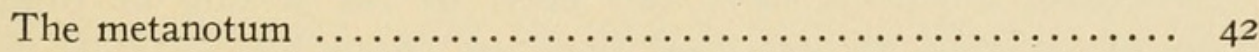

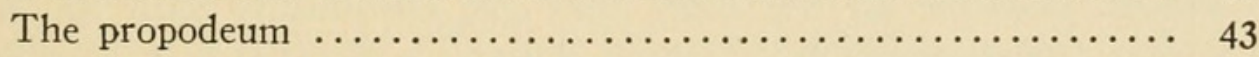

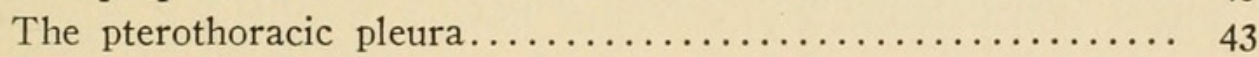

The thoracic spiracles............................ 46

The pterothoracic sternal areas...................... 48

The pterothoracic endosternum................... 49

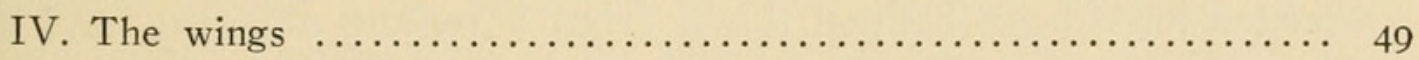

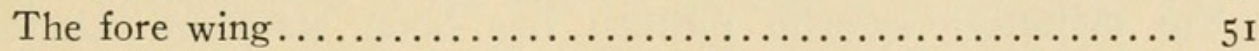

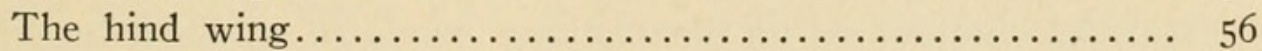

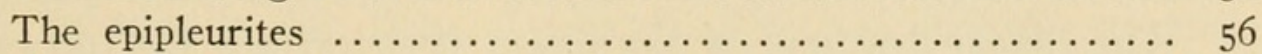

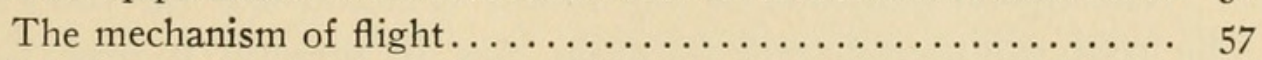

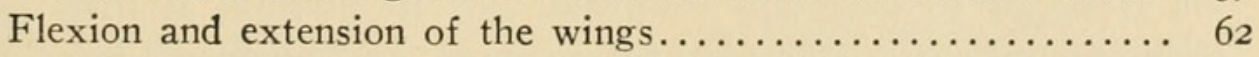




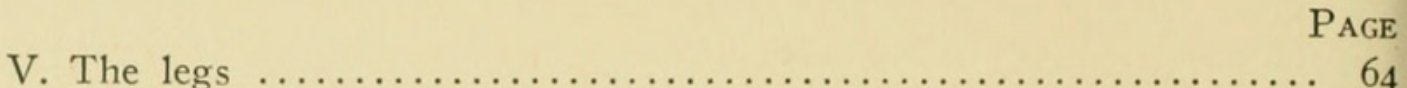

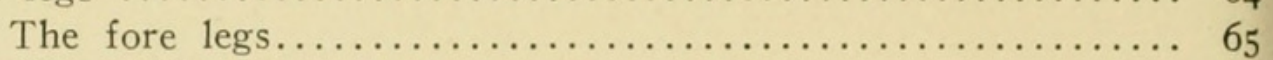

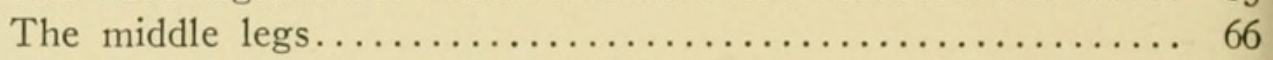

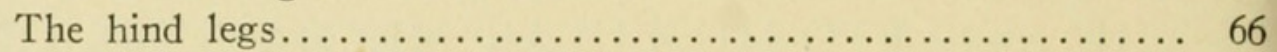

The coxae and their muscles........................ 69

The coxo-trochanteral joint...................... 72

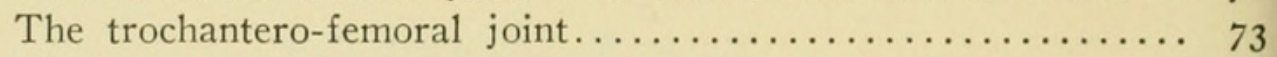

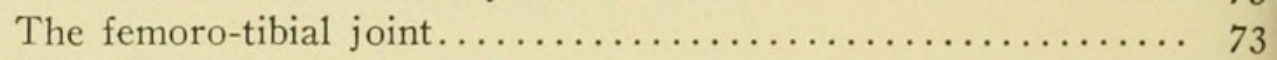

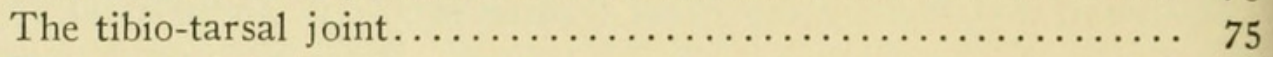

The tarsal joints.............................. 76

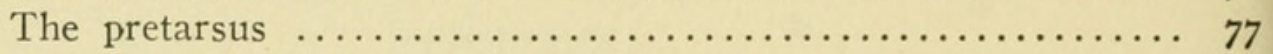

VI. The abdominal petiole $\ldots \ldots \ldots \ldots \ldots \ldots \ldots \ldots \ldots \ldots \ldots \ldots \ldots \ldots \ldots \ldots \ldots \ldots$

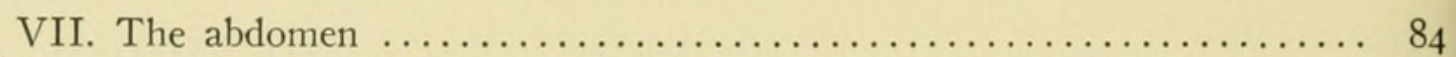

General structure of the abdomen.................... 84

The abdominal musculature............................ $8_{5}$

The male genital organ ........................... 89

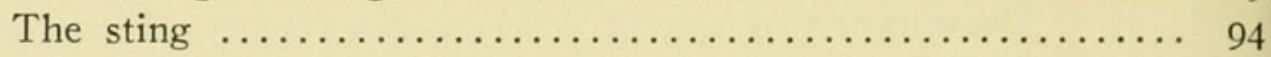

VIII. Annotated list of muscles............................ I00

Abbreviations used on the figures.................... II5

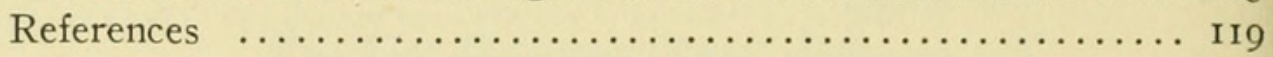

\section{INTRODUCTION}

An insect is preeminently a miniature machine; its mechanical elements are the skeleton and the muscles. The skeletal structure, therefore, cannot be fully understood without observing its correlation with the muscular system and the part it plays in the body mechanisms. And yet, almost our entire code of insect anatomy has been built up on a study of the external pattern of the skeleton, done in about the same way that a casual student of geography studies a map of the world. The results of our charting of the surface structure, however, have not been so far wrong as might be supposed, because all the lines that are commonly said to "divide" the insect exoskeleton into sclerites have a mechanical significance, and many of them are of fundamental importance in the insect mechanism. The common mistake is the assumption that the sclerites themselves are the fundamental elements of the skeletal organization, that the sclerites have been handed down from a remote ancestor, and that the primitive sclerite pattern has been somehow altered without essential changes to fit the varying needs of modern insects.

The diversifications of the insect skeleton are far too extensive to be reduced to any one formula, but in general it may be said that sclerites which are defined by grooves, or "sutures," in a continuously sclerotized region are subdivisions of the skeleton that are merely incidental to the formation of internal strengthening ridges, while 
sclerites isolated in otherwise membranous areas are themselves functional units. Most sclerites, therefore, instead of being primitive elements of the exoskeleton, are more probably recent developments accompanying the evolution of skeletal mechanisms.

With regard to the homology of sclerotized areas the question often arises as to whether or not an area of sclerotization represents necessarily a primary morphological area. As, for example, if a socalled pleural plate that ordinarily is confined to the side of the thorax is found in some species to be continued down upon the venter, are we to assume that the original pleural integument has accompanied the sclerotic extension into the venter at the expense of the primitive sternal integument, or is it merely that the physiological process of cuticular sclerotization has spread beyond the usual limits? Until this question can be answered, confusing problems in nomenclature will always intrude themselves into practical anatomical descriptions.

A similar question concerns the significance or morphological value of muscle attachments. Do muscle attachments necessarily imply homology in the skeletal parts, or can muscles shift their attachments from one place to another? In answer to this question there is abundant evidence that the so-called insertion points of muscles seldom show radical changes, while, on the other hand, the points of origin may be at widely different places on the skeleton in different species. Evidence of homology, therefore, is pretty sound when based on the working end of the muscle, though the work performed by the muscle may be entirely different in two species by reason of a change in the position of the muscle or some alteration in the skeletal mechanism. We do not know, however, whether a muscle arising at different places in two species has migrated during the evolution of one species or the other, or whether a muscle, during its formative stage of development, may evoke an entirely new attachment on the body wall by some hormonal effect at the point where its end comes in contact with the epidermis.

The musculature of the head and the feeding organs appears to be more nearly standardized than that of any other part of the insect, regardless of the great variation in structure and function of the mouth parts. The muscles of the thorax, the legs, the abdomen, and the ovipositor conform fairly well with an underlying plan, but the number of muscles is far from constant. The musculature of the male genitalia, however, shows no prevailing pattern and may be entirely different in different orders of insects. The contrast between the stability of the mouth part musculature and the variable nature 
of the male genital musculature may mean that the external genital organs of male insects, as compared with the mouth parts, are new structures having no antecedents going far back in hexapod ancestry. The wings are examples of new structures with a highly consistent musculature, but the wing mechanism has been adapted to the thoracic musculature present before wings were developed.

A study of insect mechanisms, aside from its morphological value, is important from the standpoint of understanding the functional activities of insects, and opens a wide field in entomological research to students who may be mechanically inclined. Insect mechanisms have little resemblance to man-made machines, for one reason, because animals cannot have anatomical wheels, and for another, because the insect cuticle has properties of flexibility and elasticity that react with muscle tension to produce movements that cannot be imitated in the usual rigid materials of human workmanship. The insect machine, therefore, depends on the use of levers and springs and hydraulic apparatus, but still it is not impossible that inventors might get a few hints from its study.

\section{THE HEAD AND THE FEEDING APPARATUS}

The hymenopterous head and the organs of feeding, by comparison with these parts in such orders as Lepidoptera, Diptera, and Hemiptera, show relatively little deviation from the generalized plan of structure in fundamental ways; but, on the other hand, they present numerous minor specializations and many finely adjusted mechanical alterations by which they are adapted to methods of feeding and to nonfeeding uses not found in any of the other insects. The structures directly concerned with the intake of food in the bee include the labrum, a large preoral epipharyngeal lobe on the under surface of the clypeus, the mandibles, a maxillo-labial complex, or proboscis, the mouth, and an internal sucking pump. Intimately associated with the ingestive organs, moreover, is a salivary ejection pump, or syringe, for the discharge of the saliva from the salivary duct. The hypopharynx does not appear as an independent organ in the bee; its distal part is incorporated into the base of the labium and its proximal part forms the floor of the mouth.

The natural food materials of the bee are principally pollen and nectar, both being carried to the hive for storage, where the nectar is converted into honey. The eating of pollen involves little more than a use of the mandibles in the primitive manner of biting and chewing insects, and modification of the mandibles in the bee pertain chiefly 
to the worker caste, in which the jaws serve as tools for handling wax. The ingestion of nectar from the depths of flower corollas or of honey from the cells of the comb, on the other hand, necessitates a special apparatus for reaching the liquid and for drawing it up to the mouth. Hence the principal structural modifications of the feeding apparatus affect those parts that contribute to the formation of the proboscis and the sucking pump. These same organs are able to function also in reverse for the regurgitation of nectar and honey. The principal "new" feature in the mouth parts of the bee is the salivary channel of the tongue, but this channel is probably only an elaboration of a groove along the line of union of the glossae. Finally, the need of having the proboscis out of the way when comb-building is in progress, or when the mandibles are otherwise in use, has been met by adaptations in the free parts of the proboscis that enable the latter to be folded and temporarily stored in a receptive cavity on the back of the head, from which the parts can be again extended and reassembled for functional purposes.

The so-called proboscis of the bee is hardly to be termed an "organ," since as a functional unit it is improvised when the bee would feed on liquids by bringing together the various free parts of the maxillae and the labium (fig. $3 \mathrm{~A}$ ) in such a way as to form a tube (C) through which nectar, honey, or water may be drawn up to the mouth. When not in use the parts are disassembled and folded back behind the head (fig. $3 \mathrm{~B}$ ), where they are held in place by the mandibles $(M d)$ clasped beneath them. The major parts of the maxillae and labium are adapted in form to the roles they play in the functional position; the flexing mechanism depends on smaller modifications and minute adjustments between the skeletal elements and certain muscles. All the specialized movements involved in the operation of the proboscis of the bee, however, are produced by muscles that can readily be identified with the usual muscles of the maxillae and the labium in generalized insects.

The adjustable components of the proboscis are the broad, bladelike galeal lobes of the maxillae (fig. $3 \mathrm{~A}, G a$ ), the long labial palpi $(L b P l p)$, and the slender median tongue formed of the united glossae $(G l s)$. When these elements are assembled to form a tube, the galeae and the palpi are brought alongside the tongue and, with the last, enclose a temporary channel. The wide galeae ( $\mathrm{C}, G a)$ form the sides of the tube and overlap each other above the tongue to form also the roof; the labial palpi $(L b P l p)$ lie beneath the galeae and close the tube below by underlapping the tongue, while marginal fringes of hairs on the galeae and palpi prevent leakage between 
the approximated parts. The pointed ends of the galeae converge over the protruding distal part of the tongue (fig. $3 \mathrm{C}$ ), but the small terminal segments of the labial palpi diverge laterally beyond the galeae and serve as sensory outposts. Back-and-forth movements of the tongue draw the food liquid into the canal of the proboscis, through which it ascends probably at first by capillary attraction, but before reaching the mouth the liquid is received into a preoral food channel on the base of the labium (fig. 9 A) between the bases of the maxillae, closed anteriorly by the epipharynx (B). Here at least the action of the sucking pump must become effective in finally drawing the liquid food into the mouth.

The proboscis as a whole is retractile and protractile by swinging back and forth on the long, suspensory rodlike cardines of the maxillae (figs. $3 \mathrm{~A}, 4 \mathrm{~A}, C d$ ), which are hinged to the sides of the cavity on the back of the head that lodges the bases of the maxillae and labium, but the tongue and the paraglossae themselves are deeply retractile into the end of the basal part of the labium (fig. 7 D, E).

The sucking pump of the aculeate Hymenoptera lies entirely within the head (fig. Io A), and is clearly a combination of structures that in generalized insects constitute two distinct regions of the alimentary tract. The first is the true pumping apparatus $(\mathrm{Cb})$; it is derived from the cibarial chamber of the preoral "mouth cavity," which primitively is a food pocket between the epipharyngeal wall of the clypeus and the base of the hypopharynx that is distensible by dilator muscles arising on the clypeus. The second part (Phy) represents the usual postoral pharyngeal dilatation of the stomodaeum. In the bee, as in most other sucking insects, the cibarial chamber has been enclosed within the head and converted into a sucking mechanism; but, while in Hemiptera and Diptera the pump is entirely a cibarial structure, in the Hymenoptera and Lepidoptera the cibarium and the pharynx are united to form a single pumping organ, in which, however, the cibarial and pharyngeal components may be identified. With the worker bee egestion is an important function of the feeding apparatus in the preparation of honey from nectar and in the feeding of the brood, the queen, and the drones; it is probable, therefore, that the sucking pump is also the principal regurgitative organ.

The salivary ejection apparatus of the bee is merely the salivary pocket, or salivarium, between the base of the hypopharynx and the base of the labium, into which commonly opens the salivary duct: The salivarium is converted into a closed chamber by the union of the hypopharynx with the anterior wall of the labium, and its 
hypopharyngeal and labial muscles become antagonistic dilators and compressors of the salivary chamber thus formed. The saliva presumably is conveyed around the base of the tongue within the para-
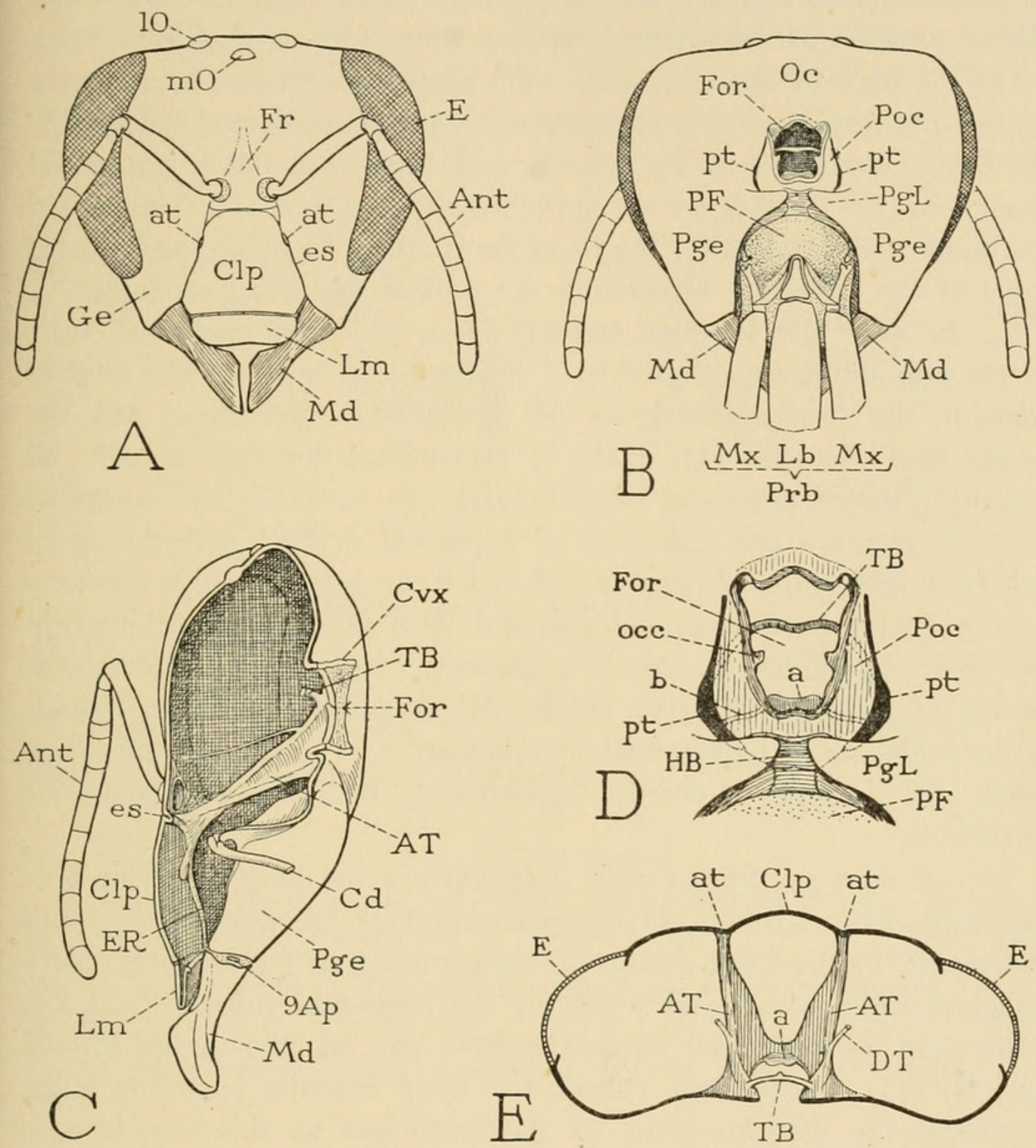

FIG. I.-The head and cranial structures of the worker.

A, anterior view of head. B, posterior view of head, with bases of labium and maxillae. C, right half of head, mesal view, showing right tentorial bar. $\mathrm{D}$, foramen magnum and associated structures, posterior view. E, horizontal section of head through upper part of foramen magnum and below bases of antennae, dorsal view.

$a$, shelflike plate on lower margin of foramen magnum; $b$, hollow bar from posterior tentorial pit $(p t)$ to plate $a$ on lower margin of foramen.

glossal lobes and carried out to the tip of the tongue through the salivary channel on the under side of the latter.

The head.-On the facial aspect of the bee's head (fig. I A) the significant feature is the unusual size of the clypeus $(C l p)$, which 
latter extends upward from the mandibles almost to the bases of the antennae beneath the strongly arched epistomal sulcus (es). The expansion of the clypeus is directly correlated with the great number of muscle fibers attached on its inner surface, which constitute the dilator muscles of the internal sucking pump (fig. Io A, C).

On the back of the head (fig. I B) the opening into the neck, the foramen magnum (For), occupies a central position, and below it is the deep excavation of the cranial wall known as the fossa of the proboscis $(P F)$, having a membranous floor in which are implanted the bases of the maxillae $(M x)$ and the labium $(L b)$. To the inflected sides of the fossa are articulated the rodlike maxillary cardines (C, $C d)$. Between the foramen and the fossa the broad postgenal walls of the cranium are connected by a subforaminal bridge. The median part of the bridge clearly is of hypostomal derivation, but the hypostomal element $(\mathrm{D}, H B)$ is constricted between a pair of mesally directed postgenal lobes $(P g L)$. In Vespidae the postgenal lobes themselves are united in a postgenal bridge, and the more primitive hypostomal bridge is reduced to an internal ridge connecting an exposed subforaminal remnant with the hypostomal margin of the fossa. Thus, as stated by Duncan (I939), the subforaminal bridge of Vespula "consists chiefly of postgenal structures, though it does include a hypostomal component." In Apis, on the other hand, the bridge is chiefly hypostomal, but includes postgenal intrusions.

At the sides of the foramen magnum are the long posterior tentorial pits (fig. I B, D, pt), from which there extends forward and downward in the head a pair of strong tentorial bars (C, E, AT) attached anteriorly on the ridge of the epistomal sulcus (C,ER), with their roots marked externally by a pair of pits in the groove (A, E, at). These bars, except for their extreme posterior ends, represent the anterior arms of the tentorium as this structure is developed in more generalized insects. Just within the foramen magnum the longitudinal tentorial bars are connected dorsally by a narrow, arched rod, or tentorial bridge (D, E, TB), and shortly before the bridge each bar gives off a slender threadlike branch representing the usual dorsal tentorial arm (C, E), which extends forward but disappears before reaching the facial wall of the head. The tentorium of the bee, besides bracing the cranial walls, gives attachment to muscles of the antennae, the maxillae, the labium, the pharynx, and the oral plate of the sucking apparatus. From the deeper part of each posterior tentorial pit there extends into the head cavity a slender hollow rod $(\mathrm{D}, b)$, which is attached mesally 
to a small plate $(a)$ inflected from the lower lip of the foramen magnum.

The antennae.-The antennae arise close together near the center of the face (fig. I A, Ant), where each appendage is implanted in a small membranous area with a slightly elevated rim, known as the antennal socket. Each antenna (fig. $2 \mathrm{~A}, \mathrm{G}$ ) is divided by an elbow into two major parts, a basal stalk, or scape (A, $S c p)$, and a long distal arm subdivided into I s small sections in the female (A) and

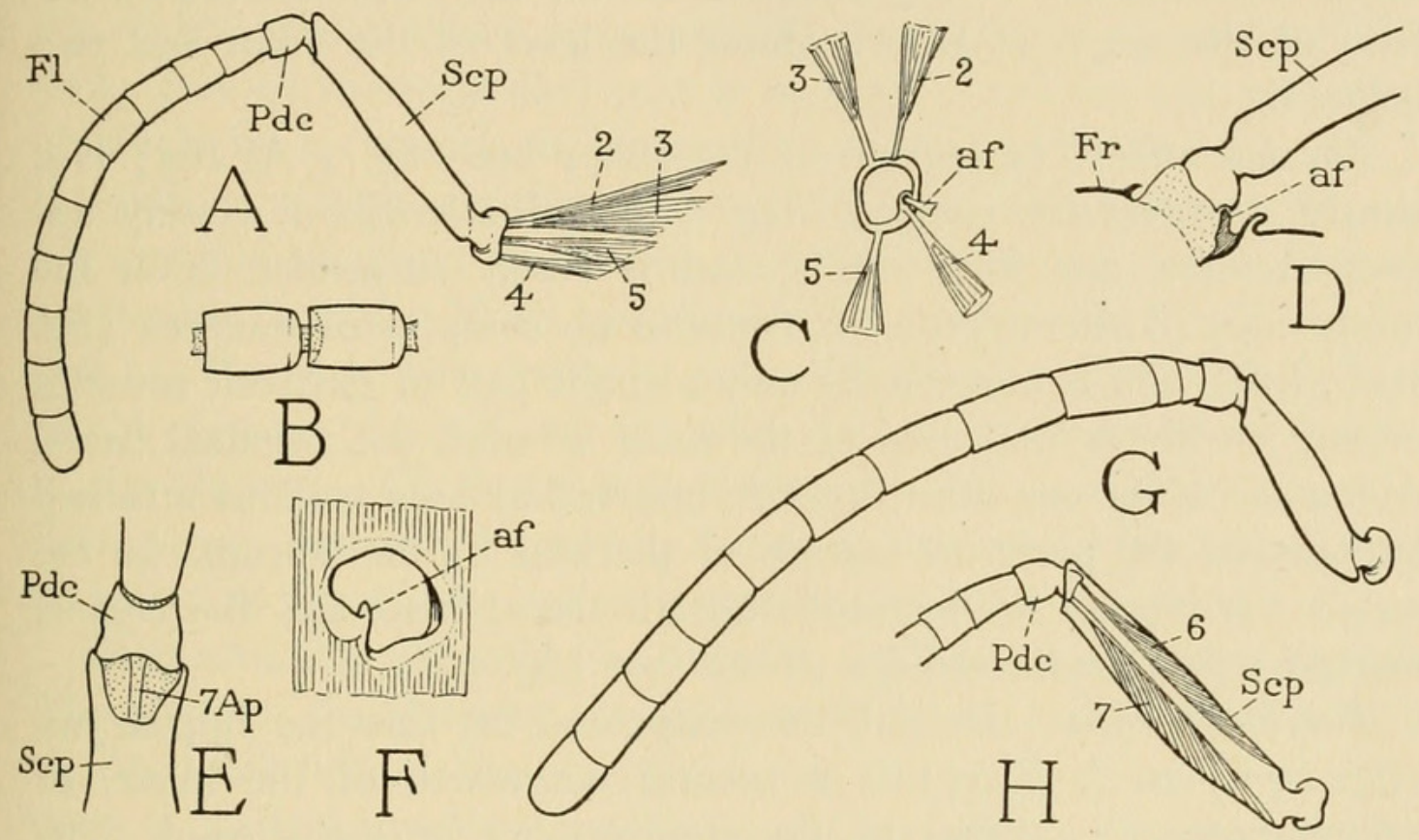

FIG. 2.-The antenna.

A, right antenna of worker and cranial muscles inserted on base, mesal view. $\mathrm{B}$, two consecutive subsegments of flagellum pulled apart to show necklike membranous connection. C, inner rim of base of right antenna, showing position of muscle insertions. D, base of right antenna and section of antennal "socket" through antennafer (af), posterior view. E, joint between scape and pedicel, ventral view. F, rim of right antennal socket, anterior view. G, antenna of drone. $\mathrm{H}$, proximal part of antenna of worker, showing muscles in scape inserted on pedicel.

I2 in the male (G). The proximal piece of the arm is usually distinguished as the pedicel $(P d c)$, and the rest termed the flagellum $(F l)$, but in the bee there is no pronounced differentiation between these two parts. The flagellar subdivisions are not true segments since they are not articulated upon each other nor provided with muscles; the rounded base of each (B) fits into a distal depression of the one proximal to it, and the two are connected by a narrow, cylindrical, necklike membrane. The joint between the pedicel and the scape, on the other hand, has a dicondylic leglike articulation (E), and hence forms a definite transverse axis of movement, on which the pedicel and flagellum are turned up or down by levator and 
depression muscles arising respectively on the dorsal and the ventral wall of the scape $(H, 6,7)$.

The scape of the antenna is articulated to the head by a distinct basal knob (fig. $2 \mathrm{D}$ ), which is pivoted on a small articular process, the antennafer $(a f)$, arising ventrolaterally from the rim of the antennal socket $(F)$. The antenna is thus freely movable in all directions except as it is limited by the socket membrane. To provide for its movement four muscles arising on the concave dorsal surface of the tentorial bar of the same side of the head are inserted on the base of the scape (C), two above the level of the pivot and two below it.

The labrum.-The labrum of the honey bee (fig. I A, $L m$ ) is a simple, transversely elongate flap with well-sclerotized, closely appressed outer and inner walls, and is freely suspended from the lower edge of the clypeus. It contains no compressor muscles (fig. Io $\mathrm{C}, L \mathrm{~m}$ ), and is movable by only a single pair of extrinsic muscles arising on the frontal area of the head between the antennal bases, which are adductors since they are inserted by long tendons attached laterally on the posterior margin of the base of the labrum. In the wasps the labrum is retracted beneath the clypeus and the clypeal margin forms the lower edge of the face (fig. $9 \mathrm{E}$ ).

The epipharynx.- In both the wasps and the bees the epipharynx (figs. $9 \mathrm{E}$, Io C, Ephy) is a preoral outgrowth of the inner, or "epipharyngeal," surface of the clypeus-not of the labrum. The epipharynx of the honey bee is a large, soft structure with a prominent median keel and padlike lateral lobes (fig. 9 A, Ephy). It is retractile by a group of divergent muscle fibers arising on the lower part of the clypeus (fig. Io C, 25). Just above and behind the base of the epipharynx is the wide, oval mouth opening (figs. $9 \mathrm{~A}$, Io C, $M t h$ ) that leads into the cavity of the sucking pump. The epipharynx is richly provided with sense organs, but mechanically it serves, together with the lacinial lobes of the maxillae, to close the food channel on the base of the proboscis (fig. $9 \mathrm{~A}, \mathrm{~B}$ ).

The mouth.-The wide aperture behind the epipharynx that leads into the sucking pump (figs. $9 \mathrm{~A}$, Io A, $M t h$ ) is the mouth of the bee in a functional sense, but since the anterior part of the pump (fig. Io $\mathrm{A}, \mathrm{Cb}$ ) represents the primitively preoral cibarium lying between the clypeus and the hypopharynx, the true mouth, in a morphological sense, is between the cibarial and pharyngeal sections of the pump. In the Hymenoptera, therefore, as in various other sucking insects, the functional mouth is a secondary constriction of the food meatus between the inner face of the clypeus and the 
anterior wall of the hypopharynx (fig. Io A, $M t h$ ). In some of the Hymenoptera the mouth is guarded by a pair of long, valvelike lips, the upper one being the epipharynx, the lower one a fold from the lower edge of the mouth. In the bee the suboral fold hangs like a bib from the lower lip of the mouth (fig. $9 \mathrm{~A}, b i b$ ).

The mandibles.-The mandibles of the honey bee differ in shape and relative size in the three castes (fig. $3 \mathrm{~F}, \mathrm{G}, \mathrm{J}$ ). In the worker (F) each mandible is elongate, thick at the base, narrowed through the middle, and widened again distally in a flattened expansion. The inner face of the expanded part is somewhat concave and traversed obliquely by a channel $(d)$ fringed on both sides with hairs, which is continuous with a groove $(e)$ that runs upward to the orifice of the mandibular gland $(f)$ at the base of the jaw. The mandible is suspended from the lower lateral angle of the cranium (fig. I A, C), and has the usual two points of articulation, one with the lower part of the clypeus (fig. $3 \mathrm{E}, c$ ), the other $\left(a^{\prime}\right)$ with the subgenal margin of the cranium. The hinge line of the jaw between the two articulations slopes downward from in front, so that when the mandible is adducted its apex turns not only mesally but also posteriorly.

Each mandible has only two muscles, an abductor and an adductor. The fibers of the abductor spread over the lower part of the side wall of the head behind the compound eye (fig. $3 \mathrm{I}, 8$ ) and converge upon a stalked apodeme arising in the articular membrane at the outer side of the mandibular base $(\mathrm{E}, 8 \mathrm{Ap})$. The large adductor muscle $(H, 9)$ has several groups of fibers, some of which arise behind the eye above the abductor fibers, others on the back of the head below the level of the foramen magnum, while a small group $(9 a)$, inserted on a long slender branch of the apodeme, takes its origin on the top of the head. The strongly stalked adductor apodeme arises from the articular membrane at the inner side of the base of the mandible (fig. I C, $9 A p$ ), and is closely applied to the mesal wall of the mandibular gland (fig. $3 \mathrm{~F}$ ).

The worker bee makes various specific uses of its mandibles, including the collecting and breaking of pollen grains for food, manipulation of wax in comb-building, supporting the base of the extended proboscis while feeding on liquids, and holding the flexed proboscis in place when the latter is folded behind the head (fig. $3 \mathrm{~B}$ ). The provision of each mandible with but two muscles gives the jaws active movements of abduction and adduction only, but there is sufficient flexibility at the articulations to allow of some play of the mandibles on each other. When the mandibles are used to hold the base of the extended proboscis, the channels on the inner surfaces 
of their expanded ends fit exactly on the midribs of the maxillary galeae and allow the latter to slide between them. When the proboscis is folded (fig. 3 B) the mandibles are crossed behind the labrum against the bases of the flexed galeae, and thus hold the proboscis snugly against the head.

Passively the mandibles, when partly opened, form a conduit for the discharge of nectar or honey and of brood food from the mouth. According to Park (I925), "the honey is forced out over the dorsal surface of the folded proboscis between the mandibles which are held well apart"; and in a personal communication Frank E. Todd of the United States Bureau of Entomology and Plant Quarantine says, "feeding of royal jelly, and of honey and pollen, to the larvae is done through the mandibles."

The mandible of the queen (fig. $3 \mathrm{~J}$ ) is of about the same length as that of the worker $(\mathrm{F})$, but is bilobed distally and much wider at the base; it has a concavity on the inner face of the apical lobe, but there is no groove leading up to the orifice of the mandibular gland. The posterior surface is clothed with more numerous and longer hairs than the worker mandible, but in both castes of the female the mandibular hairs are unbranched. The mandible of the drone $(G)$ is relatively and absolutely smaller than that of either the worker or the queen; its distal part is narrow, provided with a small apical tooth, and has a mesal depression from which a faintly marked groove leads up to the base of the jaw. The hairs of the drone mandible are particularly long and numerous, and, in contrast to those of the female mandible, are nearly all of the plumose variety.

The mandibular gland of the worker (fig. $3 \mathrm{~F}, M d G l d$ ) and the queen is a large sack lying between the facial wall of the head and the apodeme of the adductor muscle of the mandible. The gland in the worker extends upward to the level of the antennal bases, and in the queen is even larger. In the drone the mandibular gland is but a small vesicle at the base of the jaw. The secretion of the mandibular glands of the bee is said to serve for softening wax, but corresponding glands in other insects presumably have a "salivary" function in connection with feeding.

The maxillae.-The two maxillae (fig. $3 \mathrm{~A}$ ) lie at the sides of the median labium rather than before it. The long stipital sclerites (St) of the maxillary bases are implanted proximally in the membrane of the proboscis fossa on the back of the head, and are suspended from the hypostomal margins of the fossa by the slender cardines $(C d)$, which also are contained in the fossal membrane. The free distal part of each stipes bears a long, broad, tapering galea $(G a)$, a large, 

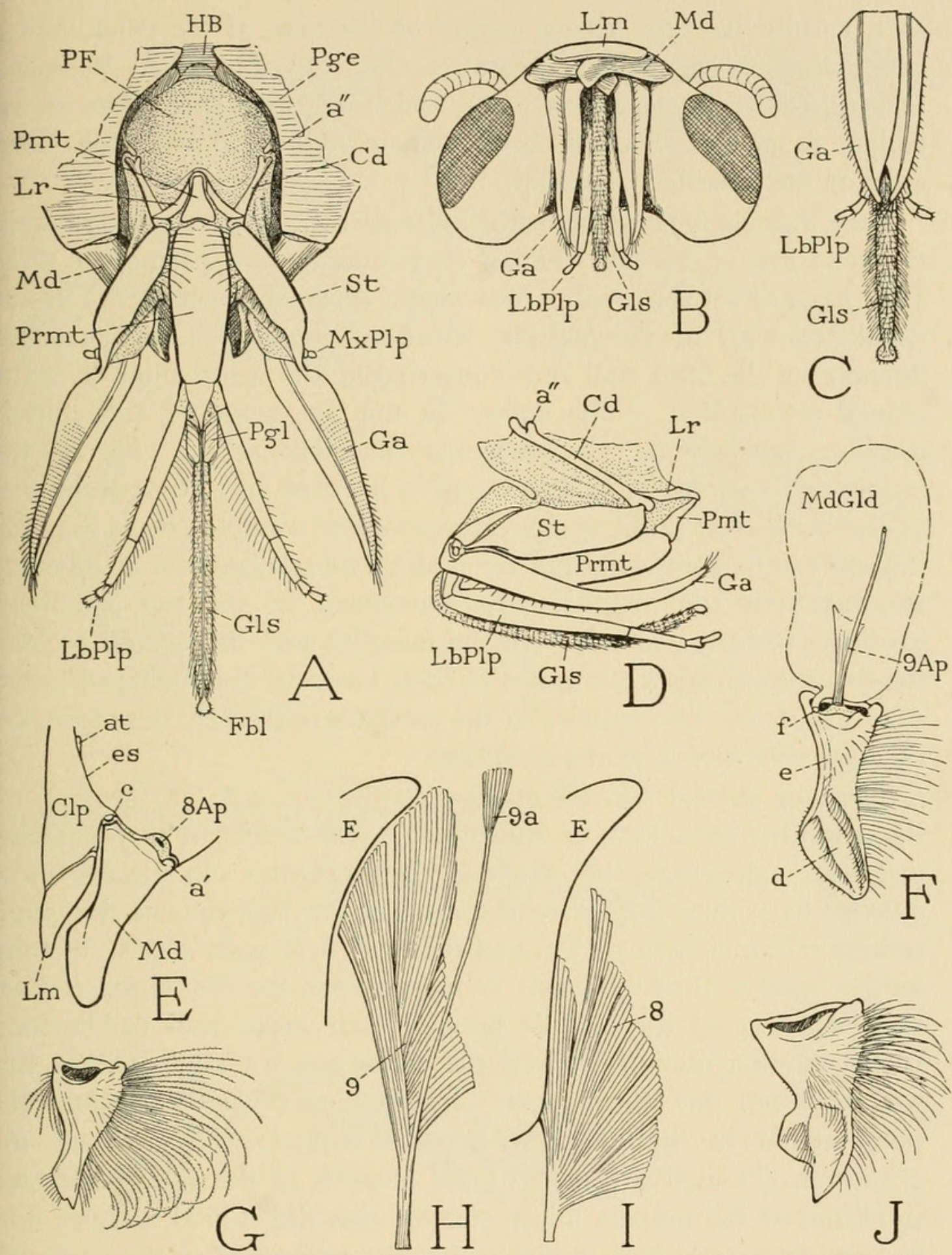

FIG. 3.-The mandibles, maxillae, and labium.

A, maxillae and labium of worker with parts artificially spread out behind the mandibles, suspended by maxillary cardines from margins of proboscis fossa $(P F)$ on back of head. $\mathrm{B}$, mandibles and free parts of maxillae and labium folded beneath head. $\mathrm{C}$, distal parts of maxillae and labium assembled to form a proboscis, anterior view. D, lateral view of retracted and folded labium and left maxilla. E, left mandible of worker and adjoining parts of head, lateral view. F, right mandible of worker and mandibular gland, mesal view. $\mathrm{G}$, right mandible of drone, mesal view. $\mathrm{H}$, adductor muscle of right mandible of worker, arising behind compound eye. I, abductor of right mandible of worker. J, right mandible of queen, mesal view.

$a^{\prime}$, posterior articulation of mandible; $a^{\prime \prime}$, cranial articulation of maxillary cardo; $c$, anterior articulation of mandible; $d$, mesal depression of mandible: $e$, mesal groove of mandible; $f$, orifice of mandibular gland. 
soft, cushionlike lobe arising mesad of the base of the galea, and a very small, 2-segmented, lateral palpus $(M x P l p)$. A V-shaped sclerite, known as the lorum $(\mathrm{Lr})$, which holds the basal plate of the labium in its apical angle, has its arms articulated with the distal ends of the maxillary cardines.

Each maxilla is provided with four extrinsic muscles arising within the head (fig. $4 \mathrm{~A}$ ), one inserted on the cardo $(I O)$; the other three $(I I, I 2, I 3)$ on the stipes. The single cardinal muscle $(I O)$ is the usual promoter of the maxilla, which in generalized insects arises dorsally on the head wall and is inserted on the cardo anterior to the cranial articulation of the latter. In the bee, however, this muscle arises posteriorly on the postgenal inflection at the side of the proboscis fossa (fig. I C, $\mathrm{Pge}$ ), and is inserted on a short lever arm of the cardo that projects above the articular condyle (fig. 4 A). By this shift in its point of origin the cardinal muscle becomes an effective protractor of the maxilla. The movement of the two maxillary cardines, however, affects both the maxillae and the labium because the three appendages are yoked at their bases by the $\mathbf{V}$-shaped lorum (fig. $3 \mathrm{~A}, L r$ ). The muscles of the cardines in the bee, therefore, are cardinal protractors of the proboscis.

The long stipital muscles of the maxilla (fig. $4 \mathrm{~A}, I I, I 2, I 3$ ) are the primitive adductors, or functionally, protractors of a generalized maxillary appendage, that arise on the tentorium. In the bee these muscles have their origins on the extreme anterior ends of the longitudinal tentorial bars $(A T)$, and hence at first sight appear to arise on the facial wall of the head. In most insects the stipital muscles act as protractors of the maxilla because their mesal pull (adduction) flattens the angulation between the cardo and the stipes and in this way protracts the appendage. The muscles evidently produce the same action in the bee, and become stipital protractors of the proboscis. Though the slant of the muscles in the fully protracted condition of the maxilla might suggest that the stipital muscles now become retractors, it is probable that the retraction of the proboscis is effected principally by the long cranial muscles of the labium (fig. $7 \mathrm{~A}, \mathrm{D}, 17)$.

The tapering bladelike galea of the maxilla is attached to the stipes by a prolongation of its base that forms a triangular plate, or subgalea (fig. $4 \mathrm{D}, \mathrm{G}, \mathrm{Sga}$ ), implanted laterally on the anterior surface of the distal end of the stipes. From the subgalea a strong midrib runs through the length of the galea to its tip. In the functional, protracted position of the proboscis the galeal blade extends straight out from 

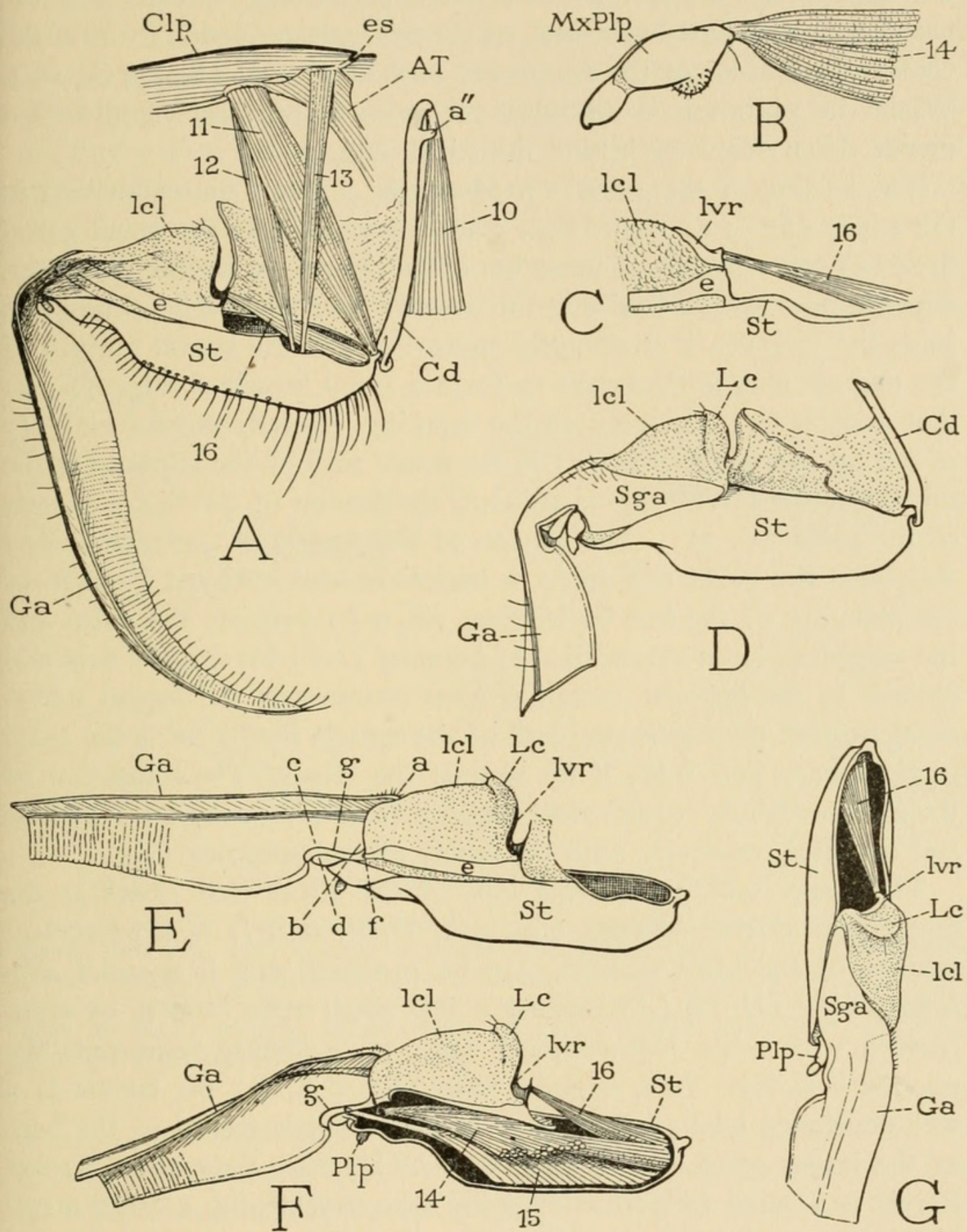

FIG. 4.-The maxilla.

A, right maxilla of worker and maxillary muscles, mesal view. B, left maxillary palpus and muscle, lateral view. C, lacinial lobe and lever of right maxilla of drone, mesal view. D, base of left maxilla of worker, lateral view. E, right maxilla with galea extended, mesal view. F, same, galea partly deflexed. $\mathrm{G}$, stipes and base of galea of right maxilla, anterior view.

$a^{\prime \prime}$, cranial articulation of cardo; $a-b, c-d$, lines of flexure in base of galea; $e$, stipital arm in base of mesal wall of lacinial lobe; $f$, articular point of galea on $e ; g$, leverlike marginal ridge on base of galea articulating with articular point $(f)$ of stipes. 
the stipes (fig. $4 \mathrm{E}$ ), its broad outer part being vertical and the narrower mesal part horizontal, as the two galeae overlap to form the roof and the sides of the temporary proboscis canal (figs. $3 \mathrm{C}, 9 \mathrm{C}$ ). When the proboscis is retracted the galea is bent back and folded upside down below or behind the stipes (fig. $3 \mathrm{D}$ ).

The folding of the galea is produced by a single muscle arising in the stipes (fig. $4 \mathrm{~F}, I_{5}$ ), which evidently represents the usual galeal flexor of other insects. The action of the muscle in the bee, however, depends on a special device in the base of the galea. Where the galea joins the subgalea it is abruptly narrowed, and the lateral margin of the narrow part is thickened to form a small leverlike ridge (E, $g$ ) that articulates proximally on the tapering, laterally curved end $(f)$ of a long narrow sclerite $(e)$ in the mesal wall of the stipes. On the middle of the lever ridge is attached the tendon of the flexor muscle of the galea $\left(\mathrm{F}, \mathrm{I}_{5}\right)$. The tension of the muscle in contraction first depresses the galea and turns it backward and outward because of the obliquity of the line of bending $(\mathrm{E}, a-b)$ between the galea and the subgalea; but a second line of bending $(c-d)$ beyond the first and oblique in the opposite direction soon counteracts the lateral movement, so that the continuing pull of the muscle finally turns the galea straight back and folds it up against the stipes. The extension of the galea evidently results automatically from the elasticity of its base and its basal continuity with the firmly affixed subgalea $(G)$.

The large, soft, cushionlike lobe of the stipes that arises at the mesal side of the subgalea (fig. $4 \mathrm{~A}, \mathrm{D}, \mathrm{F}, \mathrm{G}, l c l$ ) is a prominent feature of the bee's maxilla. On its proximal end is a small subsidiary lobe (D, G, $L c$ ) bearing a few small setae, which, by comparison with other Hymenoptera (fig. 5), evidently represents the maxillary lacinia. In a vespoid wasp (fig. $5 \mathrm{~A}, \mathrm{~B}$ ) the lacinia is a well-developed lobe $(L c)$ arising from the stipes mesad of the base of the larger galea ( $\mathrm{Ga}$ ). In Bombus (D) and Xylocopa (E) the lacinia is a small setigerous or spiny lobe overlapping a large membranous area of the stipes. It would appear, therefore, that the major part of the large cushionlike lobe of the maxilla in $A$ pis (fig. $4 \mathrm{D}, l c l$ ) is a development of the sublacinial membranous area present in Bombus and Xylocopa, and that the small setigerous lobule $(L c)$ on its base is a remnant of the lacinia proper. The entire structure in Apis is here termed the lacinial lobe; it plays an important part in the closure of the food channel on the base of the proboscis.

In the steeply declivous proximal wall of the lacinial lobe is a small curved sclerite (fig. $4 \mathrm{E}$, lvr) with a tapering distal arm and a thickened base, which latter is connected by a fulcral point with the 
proximal end of the narrow mesal sclerite $(e)$ of the stipes. The lacinial sclerite serves in the bee as a lever for keeping the lobe erect. On the upper point of its base is inserted a muscle from the stipes $(F, I 6)$, the contraction of which revolves the lever on its fulcrum and thus exerts a tension on the lobe. The lacinial lobe is the "Segelhalter" of Wolff (I875), who notes the lever sclerite in its base, and terms its muscle the "Spanner des Segelhalters." The muscle represents the flexor of the lacinia in generalized insects; the lever apparatus is present also in other Hymenoptera. In Vespula (fig. $5 \mathrm{~A}$,
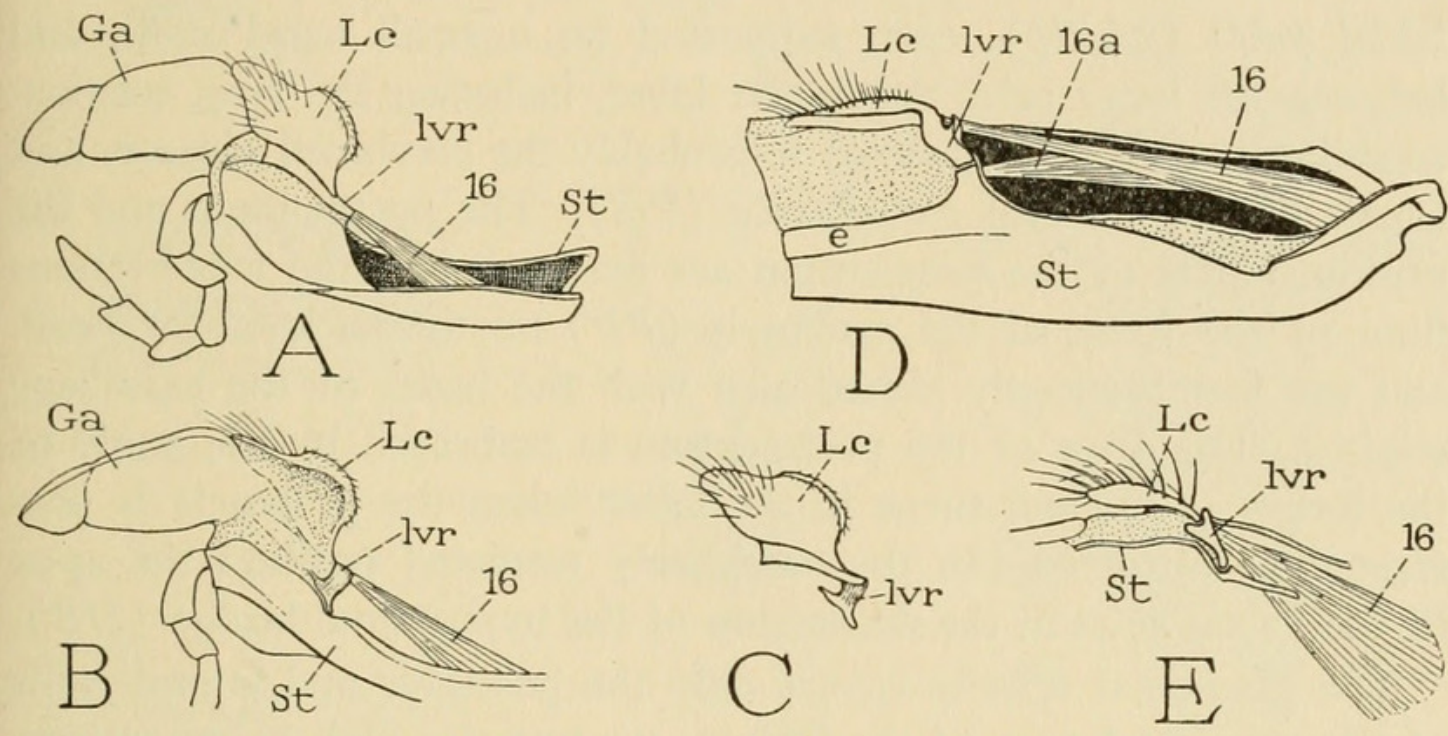

FIG. 5.-Examples of the maxillary lacinia in Vespidae and Apoidea.

A, left maxilla of Vespula maculata (L.), with well-developed lacinia, dorsolateral view. B, right maxilla of same, ventromesal view. C, detached lacinia of left maxilla of same, lateral view, showing mesal lever arm (lvr). D, base of right maxilla of Bombus americanorum (F.), with small lacinia attached to large membranous area, mesal view. E, right lacinia and lever sclerite of $X$ ylocopa virginica (L.), mesal view.

$\mathrm{B}, \mathrm{C}$ ) a small sclerite (lvr) in the base of the lacinia, giving insertion to the lacinial muscle $(\mathrm{A}, \mathrm{B}, I 6)$, curves around the proximal end of the lacinia to articulate on the stipes (B). In Bombus (D) the base of the lacinia itself articulates with the stipes, but in Xylocopa (E) there is a distinct lever sclerite (lvr) giving insertion to the lacinial muscle $(I 6)$.

The minute, two-segmented palpus of the maxilla (fig. $3 \mathrm{~A}, M x P l p$ ) arises from the end of the stipes laterad of the base of the galea, and is provided with a large muscle (fig. $4 \mathrm{~B}$ ) arising in the stipes $(\mathrm{F}$, I4). It is not evident why so small and seemingly unimportant an organ should have a muscle of such size. Wolff (1875) believed that the palpus muscle serves as an extensor of the galea, and Morison (1927) regarded it as a muscle of the galea, which, he says 
mistakenly, is attached "near" the base of the palpus. There can be no question that the muscle is inserted directly on the base of the palpus (fig. 4 B), and it is not clear how it could act as an extensor of the galea. Duncan (I939) says there are two muscles for each maxillary palpus in Vespula inserted on the palpal base.

The labium and hypopharynx.-The labium of the bee presents externally all the parts of a generalized labial appendage (fig. $3 \mathrm{~A}$ ). The lengthened base contains a small, triangular postmental sclerite $(P m t)$ and a long trough-shaped premental plate (Prmt). Diverging from the end of the prementum are the slender, four-segmented labial palpi ( $L b P l p)$, each supported on a small basal'stalk, and between the latter arise the ligular lobes, including the long, median, so-called tongue ( $\mathrm{Gls}$ ), which is probably the combined glossae, and a pair of short lateral paraglossae $(\mathrm{Pgl})$. The postmentum and the proximal part of the prementum are suspended in the membranous floor of the fossa of the proboscis $(P F)$ on the back of the head, and are membranously united also with the bases of the maxillary stipites. The apex of the postmentum is embraced by the angle of the lorum $(L r)$, and turns in the latter when the proboscis is protracted or retracted. In the completely retracted position the apex of the lorum rests in the depression of the hypostomal bridge $(H B)$.

The premental sclerite covers only the posterior and lateral walls of the premental area of the labium, the anterior wall being entirely membranous (fig. $6 \mathrm{~A}, 7 \mathrm{D}$ ). The membranous anterior wall ends in a transverse inflection at the bases of the ligular lobes (fig. 6 A), and in this groove, as may be seen by separating the paraglossae (B), is the opening of the salivary ejection apparatus $(\mathrm{SlO})$. The apparent anterior wall of the prementum, therefore, represents the hypopharynx (B, Hphy) adnate upon the labium, since the salivary duct in generalized insects opens between the hypopharynx and the labium. The hypopharyngeal surface in the bee, furthermore, extends up to the functional mouth (fig. Io A, Hphy), and includes the oral plate $(o p l)$ on the floor of the mouth cavity at the entrance to the sucking pump and the biblike fold (bib) that hangs down from the margin of the plate. The ascendent hypopharyngeal surface is flanked by a pair of slender suspensorial rods (figs. 7 D, 9 A, $s$ ) that extend from the base of the prementum to the mouth angles, and probably represent lateral hypopharyngeal sclerites of generalized insects.

The ligular lobes of the labium have a common base which is continuous with the prementum (fig. $6 \mathrm{~A}, \mathrm{~B}, \mathrm{C}$ ), but is supported on the latter anteriorly by a pair of ligular arms (A, B, $h$ ), and posteriorly by a triangular subligular plate $(\mathrm{C}, k)$. The ligular arms are sclerotic bands arising from the anterior margins of the premental plate; at 

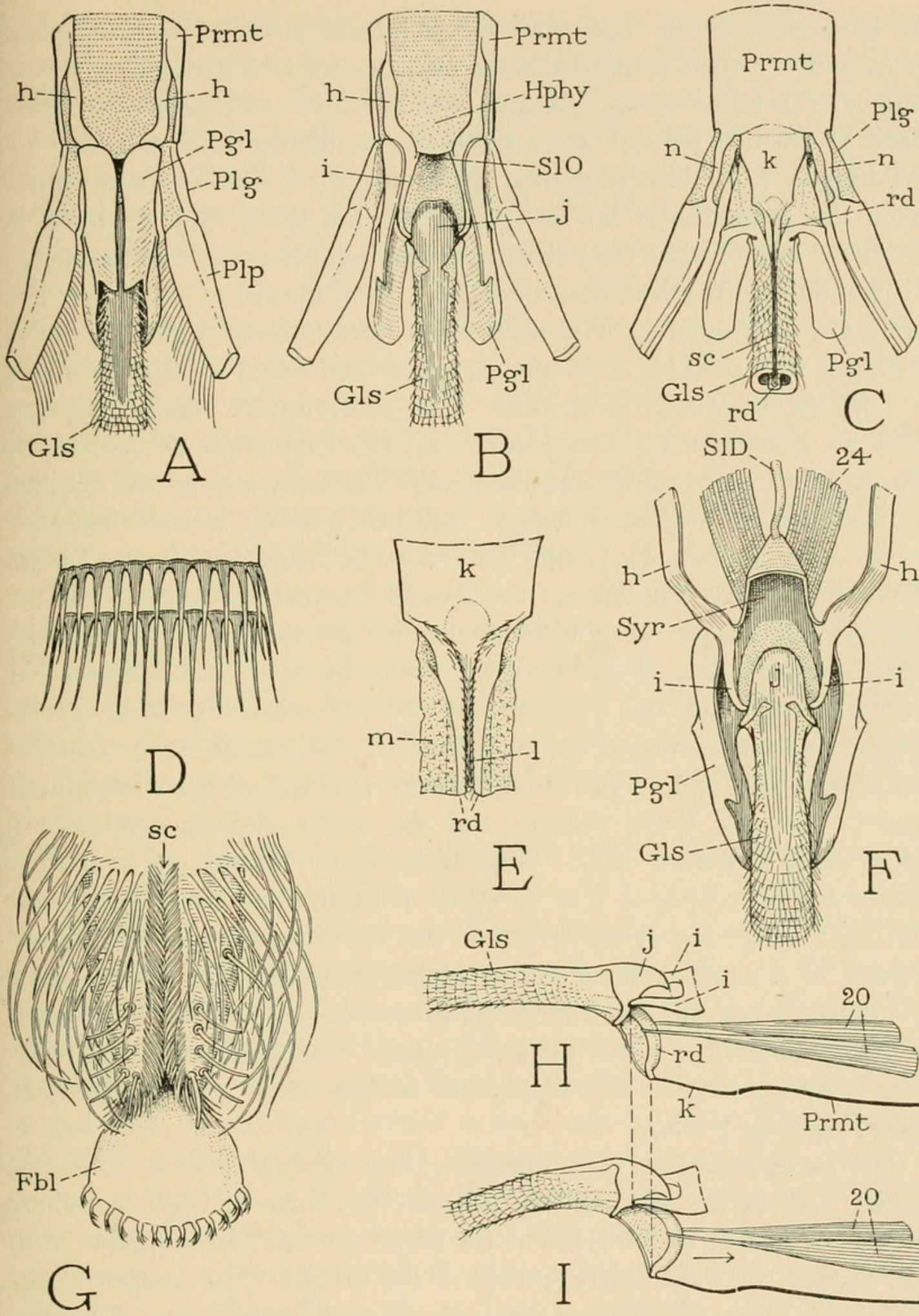

FIG. 6.-The labium of the worker.

A, end of prementum with ligula and bases of labial palpi, anterior view, paraglossae in natural position. B, same, paraglossae spread apart exposing salivary opening $(S l O)$. C, same, posterior view, showing base of glossal rod $(r d)$ and entrance to salivary channel $(s c)$. D, two tiers of glossal hairs, anterior view. E, base of glossal rod exposed by eversion of salivary channel. $\mathrm{F}$, ligula and salivary syringe, anterior view, anterior wall of syringe removed. $\mathrm{G}$, end of glossal tongue, posterior view. $\mathrm{H}$, diagram of base of protracted tongue. I, same, tongue partly retracted by contraction of glossal muscles (20).

$h$, ligular arm of prementum; $i$, pivotal process of base of tongue; $j$, basal plate of tongue; $k$, subligular plate of prementum; $l$, canal of glossal rod; $m$, membranous wall of salivary channel; $n$, elastic posterior rod of labial palpiger; $r d$, glossal rod; $s c$, salivary canal of tongue. 
the base of the ligula they are inflected laterad of the salivary opening (B), and are then continued as a pair of slender processes $(i)$ that support the bases of the paraglossae laterally, and finally curve inward to form a pair of fulcral points articulating with the base of the tongue $(j)$. The subligular plate $(\mathrm{C}, k)$ is directly continuous from the prementum, but is flexible on the latter, and its apex is produced into the rod $(r d)$ that traverses the channel of the tongue.

The long, cylindrical, densely hairy glossal tongue of the honey bee has a closely ringed structure, in which hard circles bearing the hairs alternate with membranous smooth areas (fig. 6 D), so that the organ is both flexible and contractile. The posterior, or under, surface presents a narrow median groove $(\mathrm{C}, s c)$ that expands within the tongue into a wide thin-walled channel. The inner wall of the channel is traversed by a slender, elastic rod $(r d)$, which is itself grooved on its outer surface (E), and the groove is fringed with small hairs directed distally. The lips of the glossal channel spread apart at the base of the tongue (C) and expose the proximal end of the rod, which is here seen to be directly continuous with the apex of the subligular plate $(\mathrm{C}, \mathrm{E}, k)$. Distally the rod ends in a small, freely projecting, spoon-shaped terminal lobe of the tongue, known as the flabellum $(\mathrm{G}, F b l)$. The convex under surface of the flabellum is smooth, but the distal margin and the upper surface are covered with small branched hairs. The base of the tongue is somewhat thickened; anteriorly it is covered by a hard, bonnet-shaped sclerite $(\mathrm{F}, \mathrm{H}, j$ ) decurved abruptly before the salivary orifice and produced distally in a tapering plate on the tongue surface. The basal sclerite is embraced laterally by the fulcral processes $(i)$ of the ligular arms of the prementum, which hold the tongue firmly in place, but allow it to revolve freely in a vertical plane on the transverse axis between their opposing points.

The paraglossae are thin, elongate lobes arising from the common ligular base at the sides of the tongue (fig. $6 \mathrm{~A}, \mathrm{~B}, \mathrm{C}, P g l$ ), where they are attached to the sides of the distal process of the ligular arms $(\mathrm{B}, i)$ that form the fulcral points of the tongue. The concave mesal surfaces of the paraglossae clasp the base of the tongue (A) and underlap its posterior surface, thus covering the proximal end of the tongue channel. The paraglossae automatically assume this position; they are not individually movable, since no muscles are attached directly on them.

The salivary ejection apparatus, which may be termed the salivary syringe, opens by a wide aperture situated, as already noted, anteriorly at the distal end of the prementum just behind the declivous base of 
the tongue (fig. $6 \mathrm{~B}, S l O$ ). The aperture leads into a wide, flat, triangular chamber $(\mathrm{F}, S y r$, exposed here by removal of a part of the anterior wall), into the inner end of which opens the salivary duct $(S I D)$. The floor of the chamber is a rigid, slightly concave plate continuous at its distal angles with the inflected ends of the ligular arms of the prementum $(h)$, which flank the salivary orifice (B). The roof is flexible and elastic, and gives insertion to a pair of convergent dilator muscles (fig. $7 \mathrm{~A}, 23$ ) arising on the anterior edges of the premental sclerite. A pair of much larger muscles arising in the base of the prementum (figs. $6 \mathrm{~F}, 7 \mathrm{~A}, \mathrm{C}, 24$ ) and inserted on the lateral margins of the syringe evidently act as expulsors of the saliva by flattening the dilated chamber. The salivary muscles are effective only in the protracted condition of the proboscis; as they are shown in figure $7 \mathrm{~A}$ the muscles are slack and nonfunctional because of the retraction of the ligula into the prementum.

The salivary syringe of the bee is an elaboration of the usual salivarium, which in generalized insects is a mere pocket between the hypopharynx and the base of the labium. The union of the hypopharynx with the labium has converted the salivary pocket into a closed chamber, and the ordinary hypopharyngeal and labial muscles of the salivarium become, respectively, dilators and compressors of the syringe.

The saliva, forcibly expelled from the salivary syringe, encounters at once the steeply declivous base of the tongue, which lies immediately before the salivary orifice (fig. 6 B). It must, therefore, be deflected in two divergent streams past the sides of the tongue into the concavities of the mesal surfaces of the paraglossae, and by the latter conveyed to the posterior surface of the tongue. Here, presumably, the saliva enters the tongue channel and runs through it to the tip of the organ, where it flows out on the under surface of the apical flabellum (fig. $6 \mathrm{G}$ ). It must be admitted that, so far as known to the writer, no direct observations have been made on the course of the saliva in a living bee; the relations of the structural parts concerned, however, would seem to leave no other course available than that described above, except, perhaps, that the actual conduit of the saliva through the tongue may be the groove of the glossal rod instead of the larger channel that opens on the surface of the tongue. The hair-fringed groove of the rod clearly must have some specific function.

It is possible that the same apparatus may be used also in the process of "ripening" nectar into honey, during which process, as described by Park (1925), nectar extruded from the mouth is said to 
flow over the upper surface of the proboscis and to swell into a globule in the posterior angle of the proboscis between the recurved free distal parts and the basal parts of the latter. The nectar globule is several times sucked back into the mouth and redischarged. Though recorded observations are not specific as to the exact course of the nectar around the base of the tongue, it is much to be suspected that the passageways between the tongue and the paraglossae, and likewise the glossal channel itself, the wall of which is eversible, serve to conduct the nectar in both directions.

The principal movements of the labium are (I) the back-and-forth motion of the labium with the maxillae when the entire proboscis is retracted and protracted, (2) an independent movement of the labium between the maxillae, (3) individual movements of the tongue during feeding, and (4) the backward flexion of the tongue and palpi when the proboscis is not in use. The flexion of the tongue accompanies the retraction of the proboscis, and involves a retraction of the base of the ligula into the end of the prementum. Other minor movements, probably depending on muscle tension, adjust the various parts to their functional positions.

The extrinsic musculature of the bee's labium consists of two pairs of muscles corresponding with the anterior and the posterior labial adductors of generalized insects inserted on the prementum. The anterior labial muscles in the bee (fig. $7 \mathrm{D}, I 7$ ) are the longest muscles of the head; they arise on the dorsal wall of the cranium, go downward through the head, traverse the prementum (A), and insert by tendons on the distal ends of the ligular arms $(\mathrm{A}, \mathrm{D}, h)$ of the prementum. These muscles are labial retractors of the proboscis, since their contraction affects not only the labium but the maxillae as well. Also they take part in the retraction of the ligula into the end of the prementum $(\mathrm{A}, \mathrm{E})$, which causes the deflexion of the tongue. The posterior labial muscles $(\mathrm{D}, I 8)$ arise on the anterior ends of the tentorial bars of the head and converge posteriorly to a single median tendon attached on the proximal extremity of the prementum. These muscles probably act as protractors of the labium by flattening the angle between the postmentum and prementum, thus giving the labium an independent movement between the maxillae, and in addition they draw the prementum toward the mouth during feeding. The posterior labial muscles may, therefore, be termed protractoradductors of the labium.

The movements of the tongue during feeding, or when the bee is exploring a food source, consist of lengthwise extensions and contractions of the organ, and lateral movements of the part projected 
beyond the galeae and palpi. The movements are so varied and rapid that the tongue itself seems to be endowed with mobility. Only two muscles, however, account for the activity of the tongue, and they are inserted on the base of the axial rod. These muscles (fig.
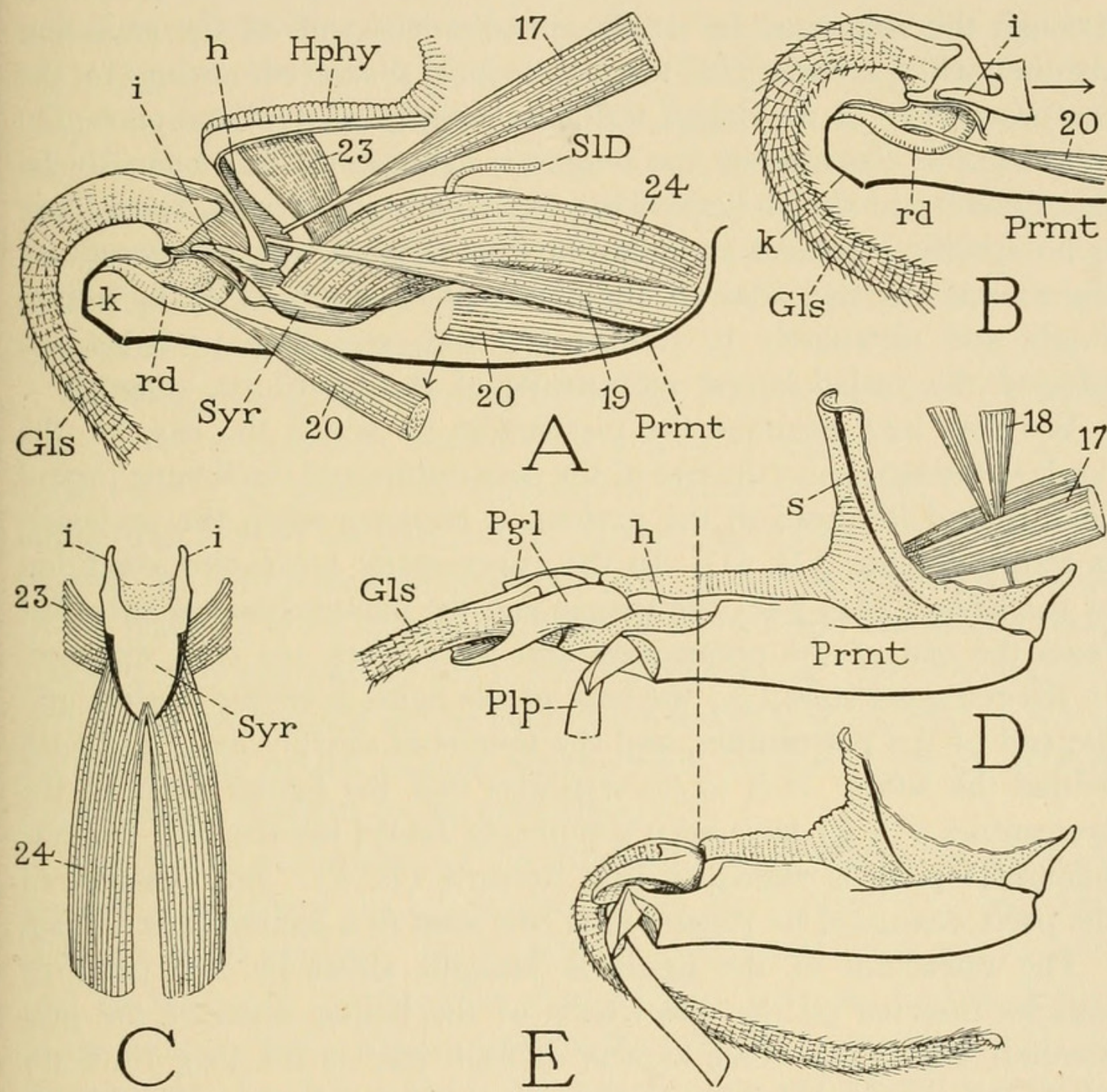

(1)

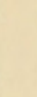


attached to the apex of the subligular plate $(k)$ at the end of the prementum. The two muscles of the rod (20) are attached by tendons, not directly on the rod, but immediately at the sides of its recurved basal part. It is clear that the contraction of the muscles increases the basal curvature of the rod (I), and therefore pulls the rod back through the tongue as far as the membranous wall of the enclosing channel will permit. The tongue is thus shortened, owing to the compressibility of its ringed wall, particularly in its distal part. On relaxation of the muscles the tongue is again extended, evidently by the elasticity of the rod and the compressed tongue wall, since there is no specific extensor mechanism. Furthermore, the attachment of the muscles at the sides of the rod enables the muscles by acting singly and alternately to revolve the rod, and thus probably are effected the varied lateral movements of the tip of the tongue.

We may now examine the mechanism by which the base of the ligula is retracted into the end of the prementum and the tongue turned back behind the head in the position it assumes when the proboscis is retracted (fig. $3 \mathrm{~B}, \mathrm{D}$ ). In the functionally protracted condition of the labium (fig. $7 \mathrm{D}$ ) the tongue and the paraglossae are extended from the end of the prementum and their bases are fully exposed. In the retracted state (E) the base of the ligula is deeply sunken into the end of the prementum, and the tongue is sharply bent backward behind the latter. It is to be seen also that the ligular arms of the prementum $(\mathrm{D}, h)$ have been completely folded inward, and that the subligular plate is angularly bent upward $(\mathrm{B}, k)$. The position of the parts concealed by retraction is best seen in a sectional view (A).

The retraction of the ligula is brought about by two pairs of muscles inserted on the distal ends of the ligular arms of the prementum (fig. 7 A). One muscle of each pair is the long retractor of the labium $(I 7)$ arising in the top of the head; the other muscle (I9) arises in the base of the prementum, and probably represents the paraglossal muscle of other insects. The pull of these muscles on the ends of the ligular supports $(h)$ inflects the latter into the membranous distal end of the prementum, and consequently retracts the attached tongue, paraglossae, and salivary syringe, while the subligular plate $(k)$ is bent forward and upward beneath the base of the tongue.

The base of the retracted tongue (fig. 7 A, B), held by the pivotal processes $(i)$ of the ligular arms, is separated by a considerable distance from the end of the subligular plate $(k)$, to which the tongue $\operatorname{rod}(r d)$ is affixed. As a consequence the rod has been drawn in a wide loop far out of the base of the tongue. Since the rod traverses 
the posterior part of the tongue in the ample membrane of the glossal channel, the contractile and flexible tongue is not only shortened by the retraction of the rod, but is sharply curved in a posterior direction. Thus the retraction of the proboscis by the retractor muscles of the labium effects also the retraction of the base of the ligula into the prementum, and the latter action produces automatically the shortening and posterior flexion of the tongue. The salivary syringe also is pulled inward by the inflection of the ligular arms, and in the retracted condition its muscles are slack and temporarily functionless. Inasmuch as there is no muscular mechanism for the protraction of the ligula, it must be assumed that the parts are extended by the elasticity of the inflected ligular arms and the subligular plate when the retractor muscles relax.

The long, 4-segmented labial palpi (fig. $8 \mathrm{~A}$ ) arise from the distal end of the prementum, where each palpus is supported on a small basal lobe $(\mathrm{Plg})$ commonly regarded as being a palpiger, i.e., a palpsupporting lobe of the prementum, rather than a basal segment of the palpus. In nonconformity with this idea, however, the single muscle of the palpus $(2 I)$ is attached within the basal lobe. The palpus itself contains only one muscle (22), which arises in the long first segment and is attached mesally on the base of the succeeding segment; it flexes the distal part of the palpus, which, when released, springs back to the extended position by its own elasticity.

The flexing mechanism of the entire palpus, by which the palpus is turned back behind the prementum when the proboscis is retracted, is in the small palpiger. Each palpiger is flattened from side to side, and its walls are membranous except for a narrow sclerotic bridge in the anterior wall, which connects the first palpus segment with the prementum (fig. $8 \mathrm{~A}$ ), and a slender, flexible and elastic rod in the posterior wall $(\mathrm{A}, \mathrm{D}, n)$ that is continuous from the prementum to the midrib of the palpus. The basal muscle of the palpus $(B, 2 I)$ is attached by a long tendon on the posterior rod a little beyond the middle of the latter. When the palpus is extended (B) the rod is slightly curved into the rear wall of the palpiger; when artificially flexed $(\mathrm{C})$ the palpus is turned back with its lateral surface posterior, and the elastic rod of the palpiger $(n)$ is strongly curved into the posterior wall of the palpiger. There can be little question, therefore, that the flexing of the palpus results, conversely, from an incurving of the rod by the pull of the muscle attached on it, and, in fact, the action can be demonstrated in a dead bee by pressing the rod so as to bend it into the palpiger. The exact movements of the palpus. 
however, depend on the obliquity of lines of bending at the ends of the palpiger (B, $a-c, b-c$, and $b-d$ ).

The movements of the palpiger can be well illustrated with a piece of stiff paper cut into the form shown at $\mathrm{E}$ of figure 8 and creased
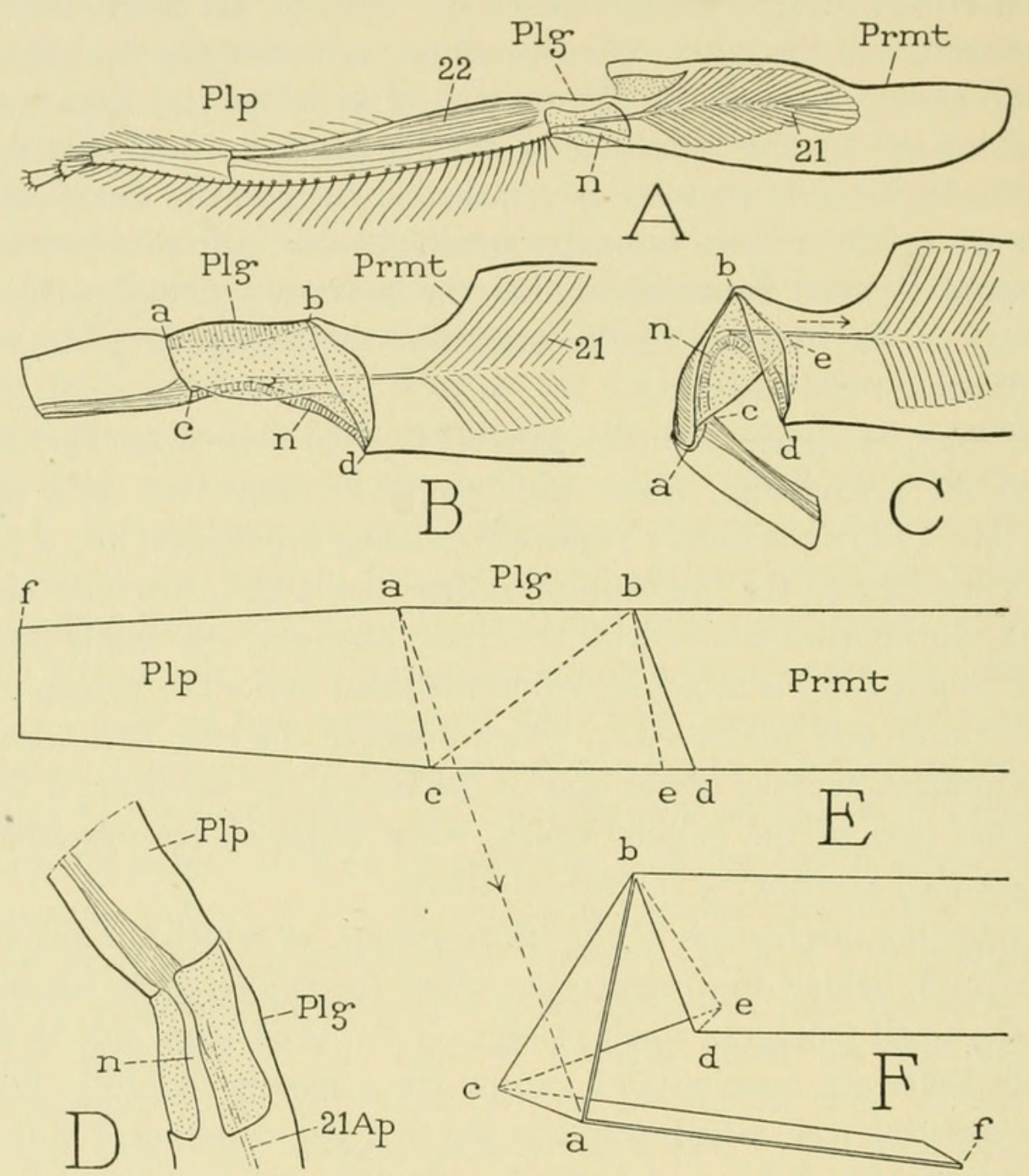

Fig. 8.-The labial palpus.

A, right palpus, palpiger, and prementum, mesal view, showing muscles. $B$, base of palpus in position of extension from prementum. C, same, palpus flexed. D, base of palpus, posterior view, showing elastic rod $(n)$ in posterior wall of palpiger, and attachment of flexor muscle tendon. E, F, diagrams of flexing of palpus on prementum, illustrated with piece of paper cut and creased as at $\mathrm{E}$, and folded as at $\mathrm{F}$.

$a-c, b-d$, lines of folding at ends of palpiger; $b-c$, line of diagonal folding of palpiger; $b-e$, line of infolding at base of flexed palpiger; $f$, distal point on palpus; $n$, elastic rod in posterior wall of palpiger.

along the lines $a-c, b-c, b-d$, and $b-e$. By folding outward along the diagonal $(b-c)$ through the "palpiger" the triangle $a b c$ is turned over and directed downward (F) ; the "palpus" can now be bent backward in a horizontal position, but its alignment with the "prementum" necessitates a compensating fold inward along the lines $b-d$ and $b-e$. 
The distance from $c$ to $d$ is thus shortened, and, by reversing the sequence of the movements, the approximation of $c$ and $d$ would produce the folding. In the palpiger of the bee (B) this shortening results from the inward curvature of the rod $(n)$ effected by the pull of the muscle on its convex side, and the palpus automatically turns over and bends backward (C). Extension of the flexed palpus evidently depends on the elasticity of the palpiger, since there is no extensor muscle.

The preoral food channel.-The membranous anterior, or hypopharyngeal, wall of the labium extends upward from the salivary orifice at the distal end of the prementum to the mouth, and forms the floor of a deep preoral food channel between the maxillary bases (fig. $9 \mathrm{~A}, F C$ ) that is continuous with the closed food canal of the distal part of the proboscis $(\mathrm{C}, f c)$. The wide aperture of the mouth $(\mathrm{A}, M t h)$ at the upper end of the channel lies behind the base of the epipharynx (Ephy), and the epipharynx is covered by the projecting labrum. On the floor of the mouth is the broad oral plate (fig. Io A, $\mathrm{B}, o p l)$, from which depends the large, thin, double-pointed, triangular fold (bib) that hangs like a bib from the lower lip of the mouth over the adoral hypopharyngeal surface (fig. $9 \mathrm{~A}, b i b$ ). At the sides of the bib in the hypopharyngeal membrane are the slender hypopharyngeal suspensory bars $(s)$, which taper downward to the sides of the prementum.

When the proboscis is depressed or retracted, the mandibles are able to close before the mouth between the labrum and the bases of the maxillae. If the mandibles are spread apart when the proboscis is protruded the preoral food canal is widely exposed (fig. 9 A) and the lacinial lobes of the maxillae $(l c l)$ are prominently displayed on the mesal margins of the stipites. When the proboscis is fully extended in the functional position, the mandibles are opened, allowing the base of the proboscis to swing up between them, and they now clasp the bases of the galeae. In this position, the proboscis extends straight out from before the mouth; the formerly vertical floor of the preoral food channel becomes more nearly horizontal, and the cushionlike lacinial lobes of the maxillae are pressed into the lateral concavities of the epipharynx, with the median keel of the latter closing the space between them (fig. 9 B), while the labrum clamps down on the epipharynx. The preoral food channel on the base of the proboscis is thus converted into a closed passageway, by which the food canal of the distal part of the proboscis is put into communication with the mouth. The approximation of the labium to the mouth bows the suspensorial rods $(A, s)$ outward and accentuates the 
depression between them into a deep heart-shaped cavity. The bib dependent from the lower lip of the mouth, however, has now assumed a nearly horizontal position with its points resting on the hypopharyngeal surface of the prementum, and thus forms a ramp from the floor of the food channel to the mouth, bridging the cavity behind it.

The cavity temporarily formed behind the suboral fold in the bee by the infolding of the adoral hypopharyngeal surface (fig. Io A, C, $I b S$ ) evidently corresponds with the permanently differentiated infrabuccal sac of certain other Hymenoptera. In Vespidae the sac is a well-developed structural feature of the preoral cavity, completely closed except for a narrow, transverse slitlike opening (fig. $9 \mathrm{D}, I b S$ ) beneath a broad suboral fold (bib) corresponding with the bib of the honey bee.

The preoral feeding mechanism of the wasps, as described by Duncan (1939) in Vespula, is similar to that of the bee, except for the more highly developed infrabuccal sac. The hypopharyngeal rods are shorter and thicker in Vespula (fig. $9 \mathrm{D}, \mathrm{E}, s$ ) than in Apis, and are termed by Duncan the "labral tractors" because when the proboscis is extended they pull down on the long basal angles of the labrum $(\mathrm{E}, \mathrm{Lm})$ and thus open the mouth. The labrum of the wasp, however, is entirely detached from the edge of the clypeus $(C l p)$ and is retracted beneath the latter. The epipharynx of the wasp, according to Duncan, has no muscles; in the bee it is provided with a group of muscle fibers arising on the clypeus (fig. Io C, 25). A short branch of each hypopharyngeal rod in Vespula (fig. $9 \mathrm{D}, \mathrm{E}, x$ ), the "tractoral suspensor" of Duncan, goes laterally to the base of the mandible, and is suggestive of being the mandibular arm of the lateral hypopharyngeal sclerite of generalized insects, on which the hypopharyngeal muscle of the mandible, when present, is attached.

Just within the mouth on the floor, or posterior wall, of the entrance to the sucking pump is the broad oral plate (fig. Io A, opl), which is commonly known as the "pharyngeal plate." There can be little doubt, however, that the plate in question is a hypopharyngeal structure, since from its proximal angles two long arms (A, B, C, y) extend upward and anteriorly in the lateral walls of the pump and give attachment to muscles arising on the frons ( $\mathrm{B}, \mathrm{C}, 32,33)$. Similar though shorter rods giving insertion to frontal muscles are present in most insects, and usually are prolongations from hypopharyngeal sclerites; the rods, together with their muscles, in fact, constitute the adductor apparatus of the hypopharynx. In some cases the pharyngeal rods are processes of a plate on the base of the 
hypopharynx, but the structure as developed in the bee (B) is characteristic of the Hymenoptera.

In the honey bee the food glands that furnish the so-called royal jelly open by pores on the distal angles of the oral plate (fig. Io B, o). It may be noted incidentally, therefore, that these glands are hypopharyngeal glands and not "pharyngeal" glands as they are generally
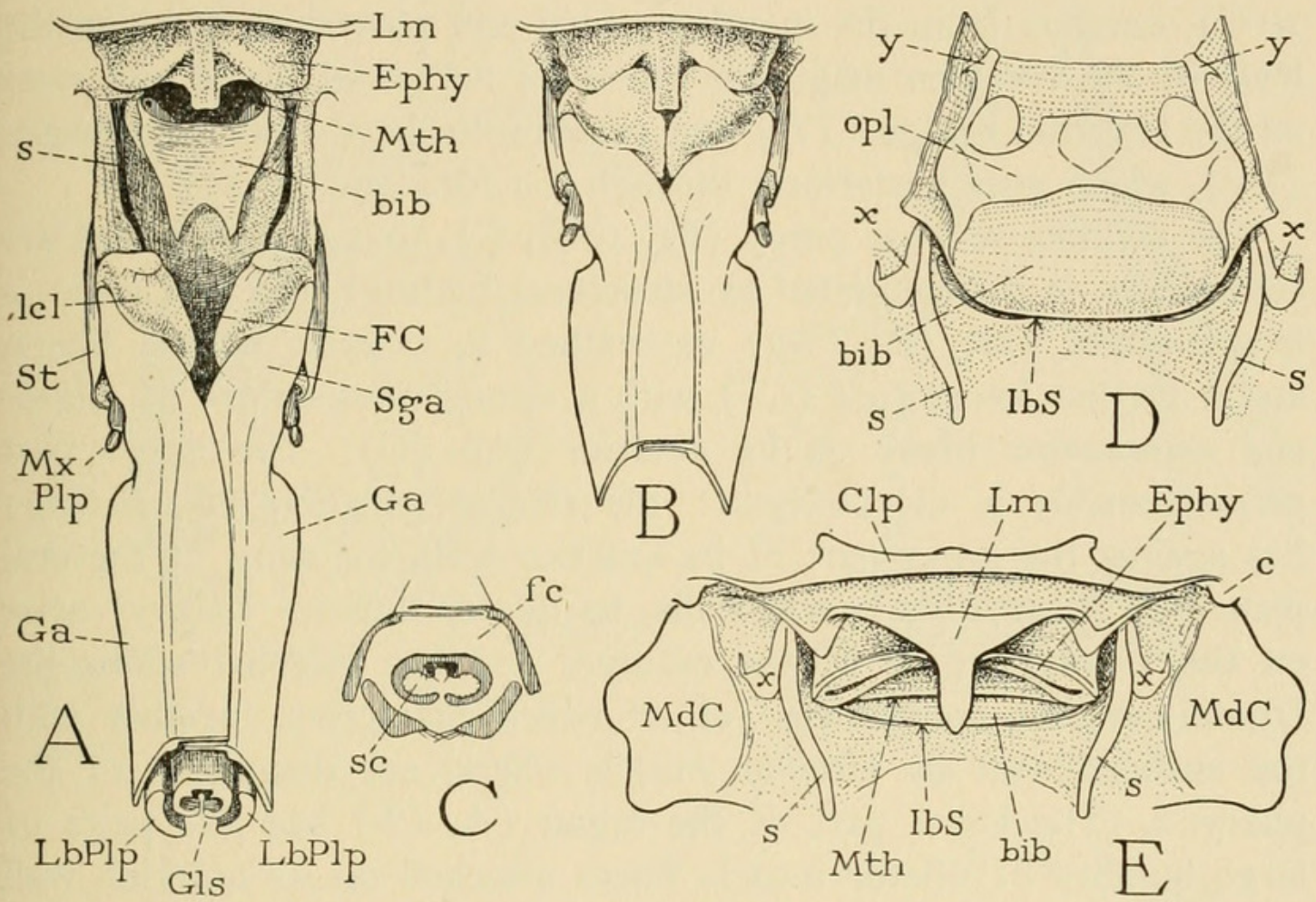

FIG. 9.-The preoral food channel and the mouth region in Apis and Vespula.

A, base of protracted proboscis of worker bee, anterior view, exposing the epipharynx, mouth, bib, and food channel between lacinial lobes of maxillae. $\mathrm{B}$, same, base of proboscis retracted, food channel closed by lacinial lobes pressed against epipharynx. C, cross section through distal part of proboscis. D, mouth region of Vespula maculata (L.), anterior wall of sucking pump removed, showing oral plate $(o p l)$, suboral bib $(b i b)$, and closed entrance to infrabuccal sac $(I b S)$. E, same in natural condition, ventral view, mouth closed to a narrow slit $(M t h)$ above closed entrance to infrabuccal sac ( $I b S)$, overhung by labrum $(L m)$.

$c$, anterior articulation of mandible; $s$, suspensorial rod of hypopharynx; $x$, mandibular branch of $s ; y$, pharyngeal arm of oral plate.

termed. Their secretion discharged upon the hypopharyngeal bib evidently accumulates in the food channel on the base of the proboscis, where it becomes accessible to other adult bees. The open food channel thus serves as a feeding trough for the queen and the drones when the latter are being fed by the workers. The muscles attached on the pharyngeal arms of the oral plate would appear to give movements to the oral plate and the bib that might facilitate the expulsion of the royal jelly into the feeding trough, but the plate with its arms 
and their muscles is present in the drones and the queen of the honey bee, and also in other Hymenoptera that do not have food glands.

The sucking pump.- The organ by which the bee draws liquids through the closed canal of the proboscis and into the mouth and passes them on to the oesophagus is a large sack with strongly muscular walls lying in the head. This sack, the sucking pump (fig. Io $\mathrm{C}$ ), extends from the mouth upward and posteriorly (A) to the level of the foramen magnum, where its narrowed upper end rests on the tentorial bridge $(T B)$ and passes into the slender oesophagus $(\mathrm{Oe})$, which goes posteriorly through the foramen.

The sacklike sucking pump (fig. Io A, C), though its outlines are continuous, is differentiated by structural features into two parts, a larger upper part (A, Phy) ensheathed in circular muscle fibers, and a smaller lower part $(\mathrm{Cb})$ with a strong musculature of dilator and compressor fibers on its anterior wall (C). The upper part unquestionably is the pharynx: the frontal ganglion (A, FrGng) lies against the lower part of its anterior wall, the arms of the oral plate $(y)$ traverse its lateral walls, its dilator muscles (dlphy) arise on the frontal region of the cranium and are inserted above the frontal-ganglion connectives - all of which characters, together with the ensheathment by circular muscle fibers, are diagnostic of the pharynx. The lower part of the organ $(\mathrm{A}, \mathrm{Cb})$ has five pairs of large bundles of dilator muscle fibers attached on its anterior wall (C, 26-30), and these muscles arise on the clypeus. This part of the pump, therefore, must be derived from the cibarium of generalized insects, which is a pocket of the preoral cavity between the under surface of the clypeus and the base of the hypopharynx. The hypopharyngeal floor of the cibarial section of the pump in the bee is represented by the oral plate $(o p l)$. In addition to the dilator muscles the cibarium is provided with thick bands of compressor fibers (C, $3^{I}$ ) running obliquely between the dilator bundles from the side of the oral plate to the median area of the anterior wall, where they turn dorsally. In addition a strong anteriormost group of compressor fibers arches over the mouth. In Hymenoptera, therefore, the sucking pump is a cibario-pharyngeal structure; in Hemiptera and Diptera the pump is purely cibarial.

The capacious pharyngeal section of the pump has no effective dilator apparatus, since only three pairs of small muscles (fig. Io C, $34,35,36)$ attach on it from the head wall. On its posterior surface two groups of long slender fibers $(38)$, arising laterally on the tentorial bridge $(T B)$, spread downward, going beneath the circular 
fibers to attach on the arms of the oral plate; and finally, a slender, fusiform, unpaired median muscle (37), arising by a long tendon on a median point of the tentorial bridge, stretches along the posterior wall and attaches on the oral plate itself.
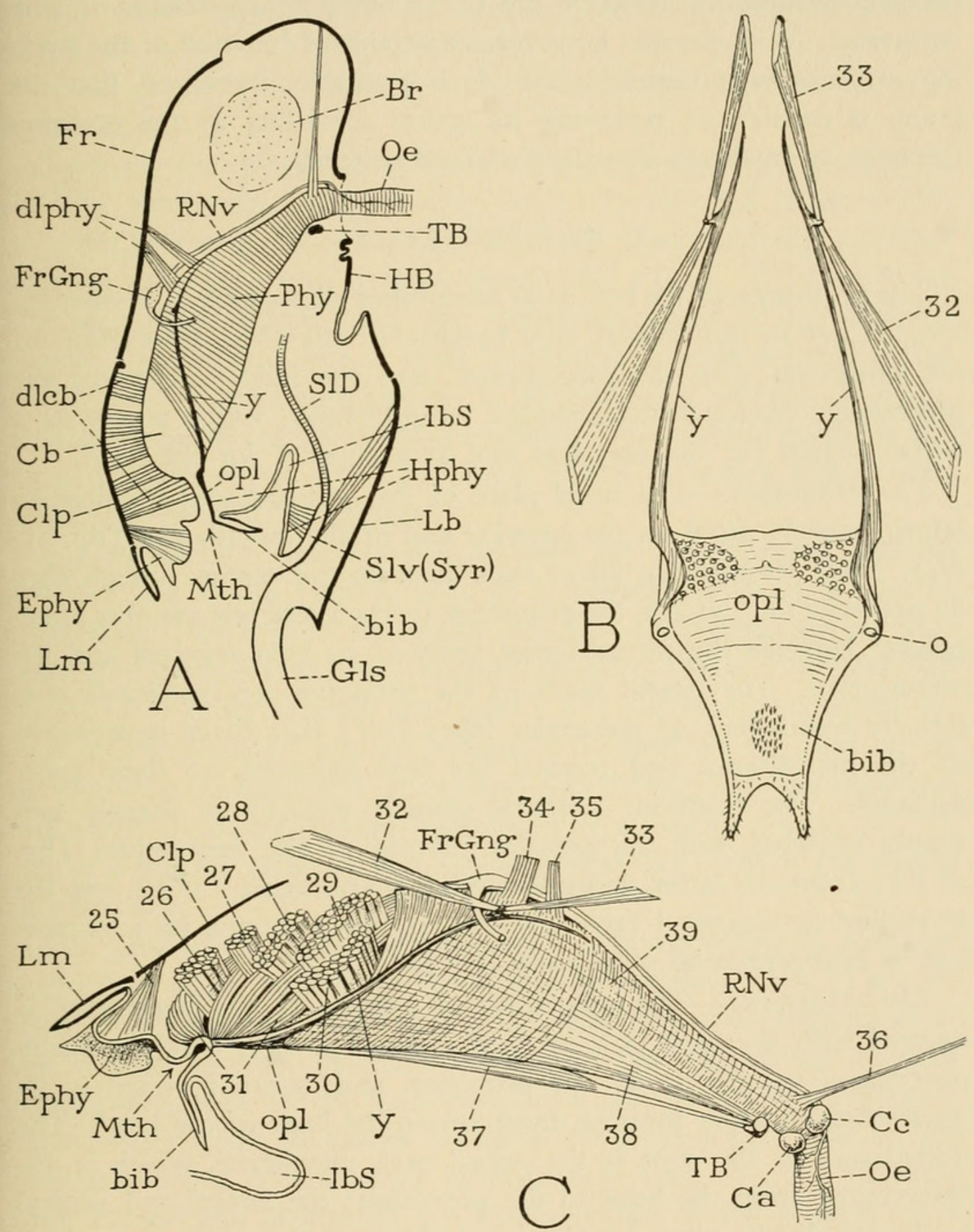

FIG. I0.-The sucking pump.

A, median vertical section of the head, diagrammatic, showing sucking pump composed of cibarial and pharyngeal sections $(C b, P h y)$, and salivary syringe $(S y r)$ in base of labium. B, oral plate, its pharyngeal arms and their muscles, and the suboral bib, flattened, anterior view. C, the sucking pump and its muscles, lateral view.

$o$, orifice of brood-food gland on distal angle of oral plate; $y$, pharyngeal arm of oral plate. 
A discussion of the working mechanism of the sucking pump without observations on the action of the organ in a living bee could be but little convincing, and will not be attempted here, except to point out that the cibarial section, with its strong equipment of dilator and compressor muscles, must be the active pumping apparatus, or true suctorium. With the bee, however, an important function of the feeding mechanism is regurgitation. It is probable, therefore, that the pump is capable of reversing its action according as the occasion demands an intake or an output of food material.

\section{THE PROTHORAX}

The prothorax of the bee, as of most other clistogastrous Hymenoptera, is not an anatomical unit because of the close association of the pronotum with the pterothorax, and the independence of the prothoracic pleurosternal complex, or propectus, which serves as a suspensorium for the first legs and as a support for the head.

The pronotum.-The notal plate of the prothorax is a collarlike sclerite closely fitted on the anterior end of the mesothorax (fig. I5, $N_{1}$ ). Since its tapering lower ends meet on the venter, though they do not unite (fig. I I A), the pronotum forms a sclerotic annulus interpolated between the pleurosternal parts of its own segment and the mesothorax. The lateral areas of the pronotum are produced posteriorly into a pair of spiracular lobes $(s p l)$ that cover depressions of the mesothorax and conceal the first spiracles on their inner surfaces. The posterior pronotal margin dorsally is sharply and deeply inflected with the anterior margin of the mesonotum (fig. II F); from the latter depends a small prephragma ( $I P h)$, but the pronotum is separated from the base of the phragma by a narrow line of intersegmental membrane $(M b)$.

The propectus.-The pleurosternal region of the prothorax is merged with the neck in a prenotal cone (fig. I5), which is partly overlapped posteriorly by the pronotum and tapers forward to its connection with the foramen magnum on the back of the head. The dorsal wall of the cone is the dorsal neck membrane, which is uninterrupted from the head to the pronotum; the lateral and ventral walls contain the pleural and sternal plates of the prothorax, which together constitute the propectus (fig. II G).

Each prothoracic pleuron is an elongate triangular plate (fig. II C), the anterior apical part of which tapers into a mesally bent occipital process $(\mathrm{C}, \mathrm{E}, e)$ that articulates with the corresponding occipital condyle of the head (B), and which, therefore, would appear to 
represent a cervical sclerite fused with the pleuron. On the lower posterior angle of the pleural plate is articulated the coxa of the first leg $(C, C x)$. From the coxal articulation there extends upward close to the posterior pleural margin a groove $(p l s)$, which is the pleural sulcus, since it runs into the base of the large internal pleural apophysis $(P l A)$. The groove sets off a very narrow posterior epimeral strip $(E p m)$ of the pleuron from a large anterior episternal region $(E p s)$.

Internally the episternum presents close to its upper margin a wide, shelflike horizontal apodeme (fig. II E, c) inflected from a submarginal groove on its outer surface $(\mathrm{C}, b)$. The horizontal apodeme expands anteriorly into a large mesal lobe $(\mathrm{C}, \mathrm{E}, d)$, termed by Duncan (1939) in the wasp the "posterior ramus of the occipital process," but which, since it gives attachment to several muscles that are in most insects inserted on a distinct neck sclerite, may appropriately be named the cervical apodeme. The cervical apodeme and the pleural apophysis form prominent points of muscle attachments at opposite ends of the pleuron.

The prosternum lies ventrally between the pleural plates and the bases of the coxae (fig. II G). It is differentiated by a narrow constriction into a wide, triangular, anterior basisternal region (Bs) flanked by the episterna, and a narrow, elongate, posterior furcasternal region (Fs) lying between the coxae. The furcasternum bends upward at a steep angle from the horizontal basisternum (I), and carries an elaborate endoskeletal structure, the prothoracic endosternum. Externally the furcasternum is featured by a median groove $(K, f)$, and by a pair of posterior pits $(g)$ that mark the roots of the sternal apophyses.

The prothoracic endosternum.-The large endoskeletal structure supported on the sternum of the prothorax occupies much of the interior of the segment (fig. II B, E, Endst) and gives attachment to various muscles (fig. I2 B, C). Structurally the endosternum includes a supporting base on the furcasternum, a pair of laterally divergent dorsal wings, and a horizontal bridge between the wings. The sternal support consists of a vertical median plate (fig. I I I, $h$ ) inflected from the median groove of the furcasternum, which posteriorly is reinforced by a pair of lateral thickenings $(i)$ arising from the posterior furcasternal pits, and anteriorly tapers into a low ridge that runs out on the basisternum $(j)$. The endosternal wings are a pair of plates with thickened anterior margins diverging from the basal ridge $(\mathrm{J}, k, k)$ in a forward direction because of the upward slant of the furcasternum in the natural position (I). The bridge is a wide, 

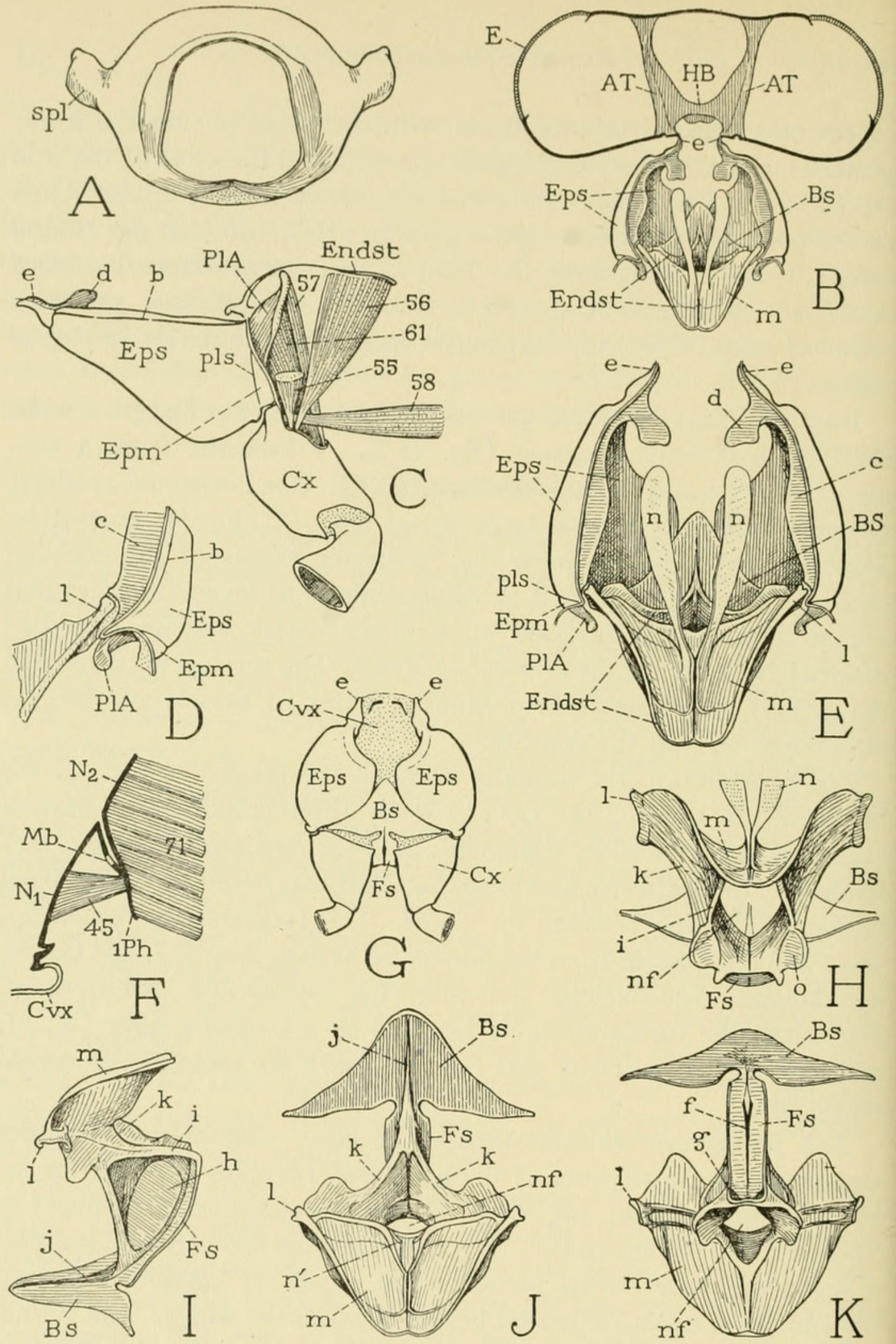

FIG. II.-The prothorax (drone).

A, pronotum, anterior view. B, pleurosternal parts of prothorax and horizontal section of attached head, dorsal view. C, propleuron and base of first leg, left side. D, articula. tion of lateral arm of endosternum with propleuron, dorsal view. E, pleurosternal and endosternal skeleton of prothorax, dorsal view. F, median section of pronotum and adjacent part of mesonotum, showing first phragma $(I P h)$ to be a prephragma of mesonotum, separated from pronotum by narrow membrane $(M b)$. G, pleurosternal parts of prothorax and bases of first legs, ventral view. H, prosternum and endosternum, posterior view. I, same, natural position, lateral view. J, same, anterodorsal view. $\mathrm{K}$, same, ventral view, furcasternum horizontal.

$b$, submarginal groove of episternum; $c$, horizontal apodeme of episternum along line of external groove $b ; d$, cervical apodeme of episternum; $e$, occipital process of episternum; $f$, median groove of furcasternum; $g$, posterior pit of furcasternum; $h$, median basal plate of endosternum; $i$, thickened posterior edge of wing plate of endosternum; $j$, median ridge of basisternum; $k, k$, divergent wing plates of endosternum; $l$, lateral extremity of endosternum; $m$, supraneural bridge of endosternum; $n$, apodeme of muscle $5 I ; n^{\prime}$, base of $n$, cut off; 0 , attachment lobe of muscle 52 . 
scoop-shaped plate, broad anteriorly, tapering and rounded posteriorly $(\mathrm{I}, \mathrm{J}, m)$, set approximately horizontally on the upper edges of the wing plates. The space between the wings is thus converted by the bridge into a short channel, which gives passage to the nerve cords, and hence may be termed the neural foramen $(\mathrm{H}, \mathrm{J}, \mathrm{K}, n f)$. The lateral extremities of the bridge $(l)$ are closely articulated with the posterior angles of the horizontal apodemes of the episterna (D, E).

The prothoracic endosternum of the bee does not have the structure of an ordinary $Y$-shaped sternal "furca," in which the divergent arms are the primary sternal apophyses carried inward by a median sternal inflection that forms the stalk of the fork. In the bee the posterior lateral thickenings that brace the median plate (fig. II, H, I, $i$ ) appear to be independent ingrowths from the furcasternum and evidently represent the usual pair of sternal apophyses, which, while retaining their bases on the sternum, have united with the posterior end of the median plate. The supraneural bridge may be regarded as a secondary formation produced by the union of flat mesal outgrowths from the divergent wing plates.

Various aspects of the prothoracic endosternum are shown in figure II. At $B$ and $E$ the endosternum is seen in place within the prothorax, with its lateral extremities articulating against the pleura. $\mathrm{H}$ gives a posterior view looking through the neural foramen, only the posterior end of the upturned furcasternum being visible. At I the endosternum and sternum are shown from the left side, the basisternum being horizontal, the furcasternum steeply ascending. J gives an anterodorsal view of the same parts, showing the endosternal wings diverging upward from the median basal ridge and united by the supraneural bridge. At $\mathrm{K}$ the sternum and endosternum are shown from below as seen when the furcasternum is turned horizontally.

The prothoracic muscles.-The musculature of the prothorax comprises $(I)$ muscles that move the head directly, (2) dorsal and ventral intersegmental muscles between the prothorax and the mesothorax, (3) muscles that move the propleura, or the entire propectus, which probably effect indirectly movements of the head, and (4) muscles of the legs.

The direct muscles of the head are attached on the margins of the foramen magnum, not in the usual manner on a postoccipital ridge. They include four pairs of levator muscles and one pair of depressor muscles. Two pairs of the levators take their origins on the prephragma of the mesothorax (fig. I2 A), one pair (40) being median, parallel, and inserted on the head laterad of the apical notch of the foramen magnum $(\mathrm{B}, 40)$, the other pair $(\mathrm{A}, 4 I)$ lateral in origin, 
convergent beneath the first, and inserted in the apical notch of the foramen $(\mathrm{B}, 4 I)$. The third pair of levators are large three-branched muscles arising by broad bases on the episterna $(\mathrm{D}, 42)$, one branch of each muscle (42a) above the horizontal apodeme of the episternum $(c)$, the second $(42 \mathrm{~b})$ on the side below the apodeme, the third $(42 \mathrm{c})$ on the ventral expansion; the three branches converge to a strong tendon attached in the laterodorsal notch of the foramen $(B, 42 a$,
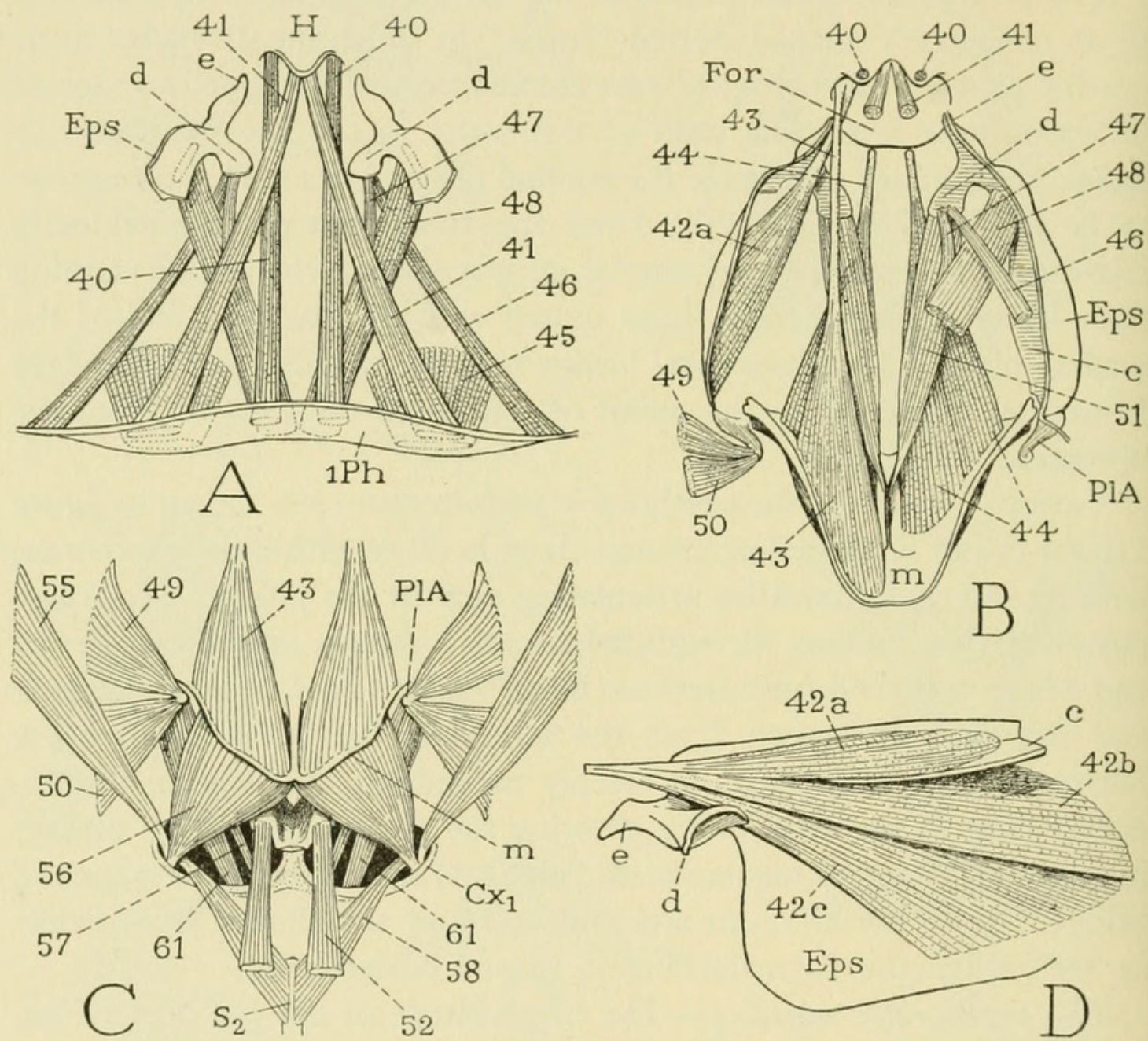

FIG. I2.-Muscles of prothorax (drone).

A, dorsal muscles, ventral view. B, ventral and lateral muscles, dorsal view. $\mathrm{C}$, muscles of endosternum and fore coxae, posterior view. D, branches of cephalic muscle 42 arising on inner face of proepisternum.

$c$, horizontal apodeme of episternum; $d$, cervical apodeme of episternum; $e$, occipital process of episternum; $m$, supraneural bridge of endosternum.

only one branch, on the left side, shown in this figure). The fourth pair of head levators arises posteriorly on the dorsal surface of the supraneural bridge of the endosternum (B, C, 43), the fibers of each muscle converging to a long, thick tendon attached on the head just laterad of the pleural levator $(\mathrm{B}, 43)$. The depressors of the head are a pair of large two-branched muscles $(\mathrm{B}, 44)$ arising on the 
supraneural bridge beneath the endosternal levators (43), and inserted by strong tendons on the lower margin of the foramen magnum. Duncan (I939) describes the two branches of this muscle in Vespula as first and second depressors of the head, but adds that the two might be considered subdivisions of a single muscle.
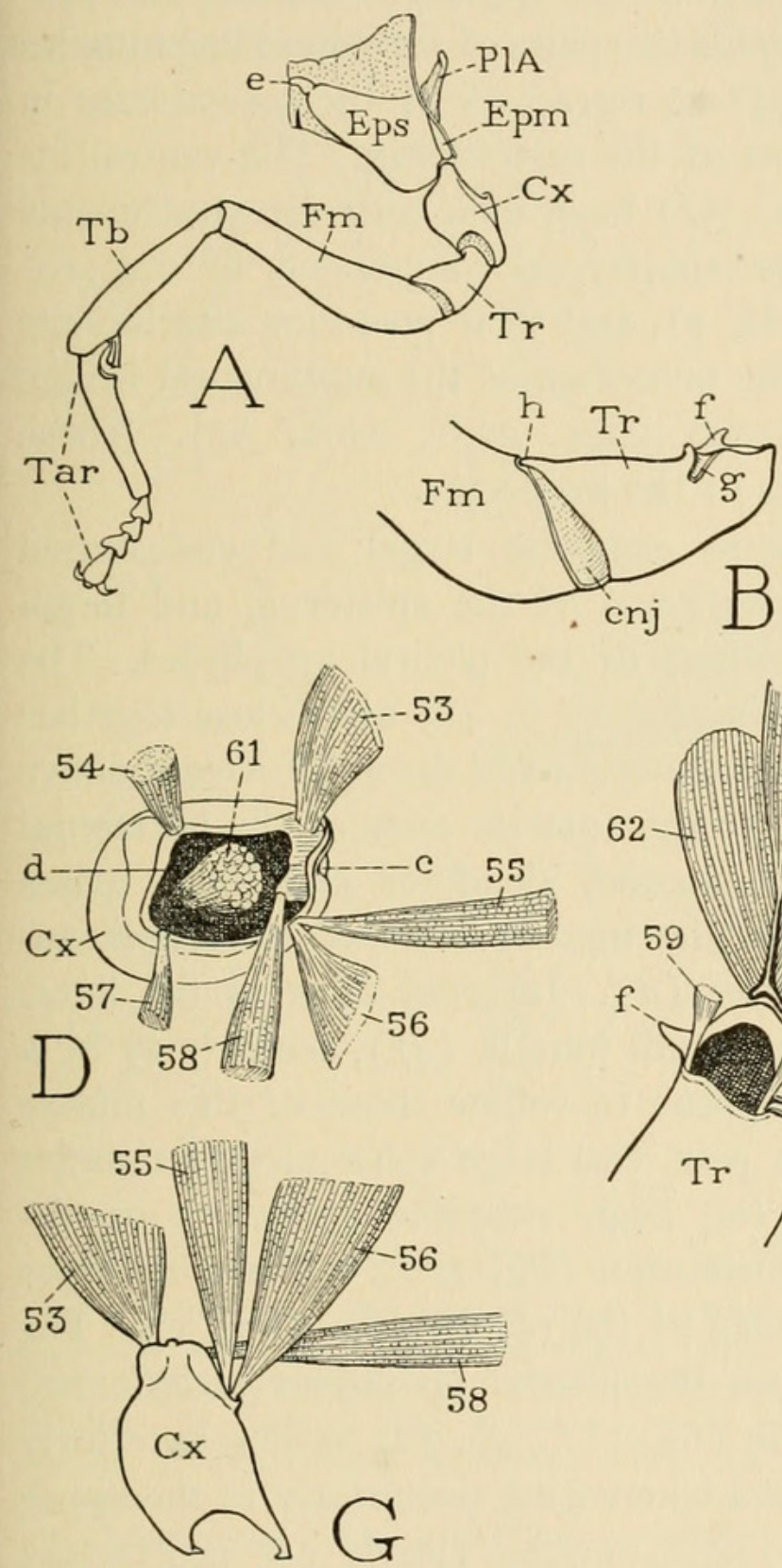
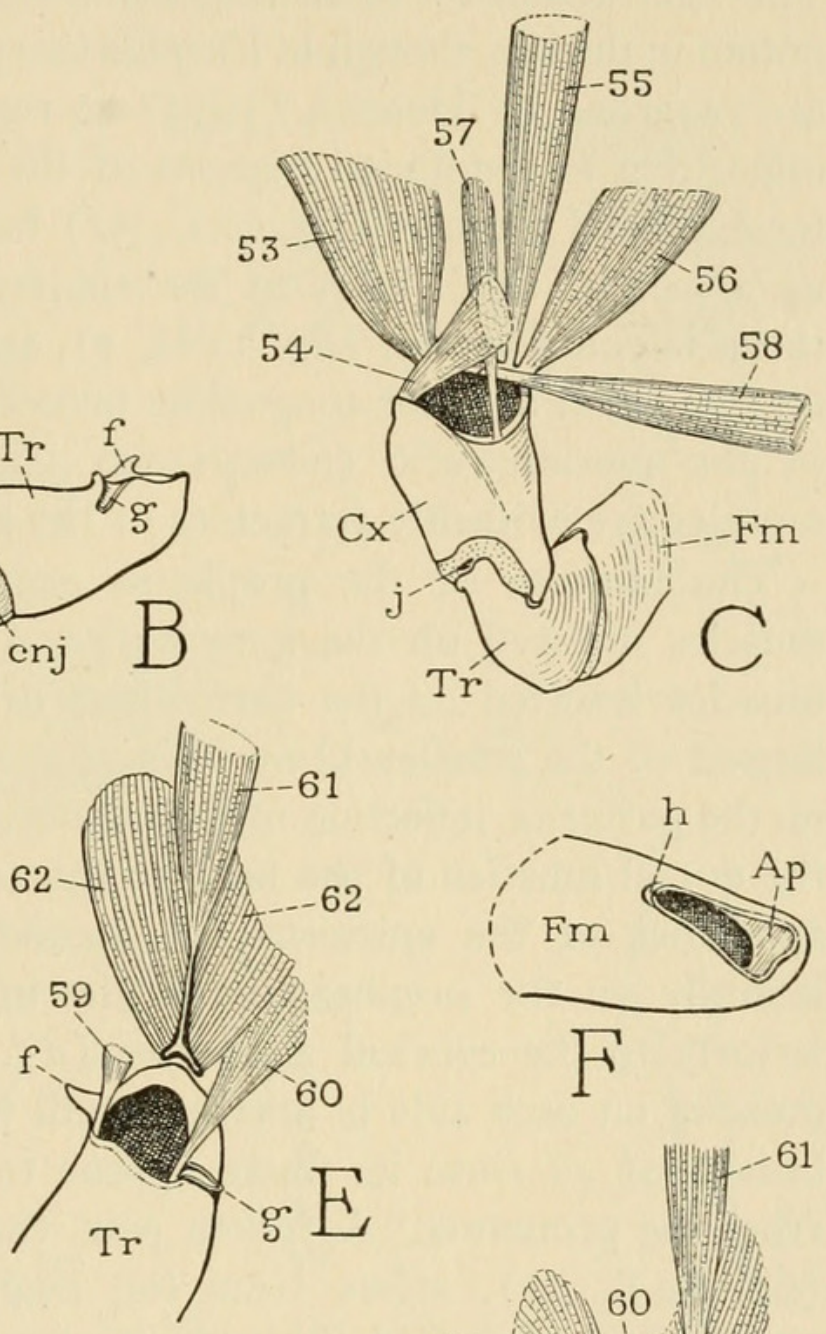

FIG. I3.-The prothoracic leg (drone).

A, left half of propleuron, and left fore leg, lateral view. B, trochanter and base of femur of left leg, posterior view. C, base of right leg and coxal muscles, mesal view. D, base of right coxa with muscles, dorsal view. E, base of left trochanter with trochanteral muscles, dorsal view. F, base of right femur, anterodorsal view. G, left coxa with lateral muscles, lateral view. $H$, right trochanter and base of femur with muscles, anterior view.

$c-d$, axis of pleurocoxal articulation; $e$, occipital process of episternum; $f$, anterior coxo-trochanteral articulation; $g$, posterior coxo-trochanteral articulation; $h$, dorsal trochantero-femoral articulation; $j$, base of depressor apodeme of trochanter. 
The dorsal and ventral intersegmental muscles of the prothorax are, respectively, intertergal and intersternal. The dorsal muscles are two large bundles of fibers (fig. I2 A, 45) arising anteriorly by broad bases on the pronotum (fig. I I F), and tapering somewhat to their posterior attachments on the prephragma of the mesonotum ( $I P h)$. The contraction of these muscles would appear to depress the pronotum in the bee, though in Vespula two pairs of corresponding muscles are regarded by Duncan (1939) as retractors of the mesonotum in opposition to the dorsal muscles of the mesothorax. The ventral intersegmental muscles (fig. I2 C, 52) have their anterior attachments on a pair of small lobes of the apophyseal components of the prothoracic endosternum (fig. I I $\mathrm{H}, o$ ), and their posterior attachments on two small anterior tonguelike processes of the supraneural bridge of the pterothoracic endosternum (figs. $22 \mathrm{C}, 24 \mathrm{~A}, 5^{2}$ ). These muscles are evidently retractors of the propectus.

The muscles of the propleura comprise tergal and endosternal muscles inserted on the anterior parts of the episterna, and tergal muscles inserted on the extremities of the pleural apophyses. The largest of the propleural muscles (fig. I2 A, 48) arise close together on the posterior inflection of the pronotum and diverge forward above the dorsal muscles of the head to the anterior ends of the horizontal apodemes of the episterna. A second, slenderer pair (46) arises laterally on the prephragma of the mesonotum and converges anteriorly to the cervical apodemes $(d)$. Inserted close to the latter muscles on each side is another small muscle (47), which may be a branch of 48 since its fibers appear to follow those of this muscle from the pronotum. A fourth pair, two large sternopleural muscles (fig. I2 $\mathrm{B}, 5^{I}$ ), arises from the long, straplike apodemes of the supraneural bridge of the endosternum (fig. I I E, $n$ ), and diverges forward to the cervical apodemes of the pleura (fig. I2 B, $d$ ). The tergopleural muscles inserted on the pleural apophyses include two fan-shaped muscles on each side (fig. I2 C, 49, 50) arising anteriorly on the side of the pronotum, and converging posteriorly to the upper extremity of the apophysis. These muscles clearly are protractors of the propectus, their antagonists being the ventral intersegmental muscles $\left(5^{2}\right)$.

The strength of the anterior pleural musculature of the prothorax suggests that movement of the pleural plates has some important purpose. The head, being articulated on a transverse axis between the occipital processes of the episterna (fig. I I B), can have only a tilting movement on the thorax by the action of its own levator and depressor muscles; but the pleural plates, by reason of their lack of fixity on the 
sternum, their posterior articulations on the endosternal arms, and the shortness of the occipital axis between their anterior ends (fig. II B, $e$ ), are well adapted to give partial rotary movements to the head by a differential, or antagonistic, action of the right and left sets of muscles attached on their anterior parts.

The prothoracic leg muscles will be described in connection with the structure of the legs.
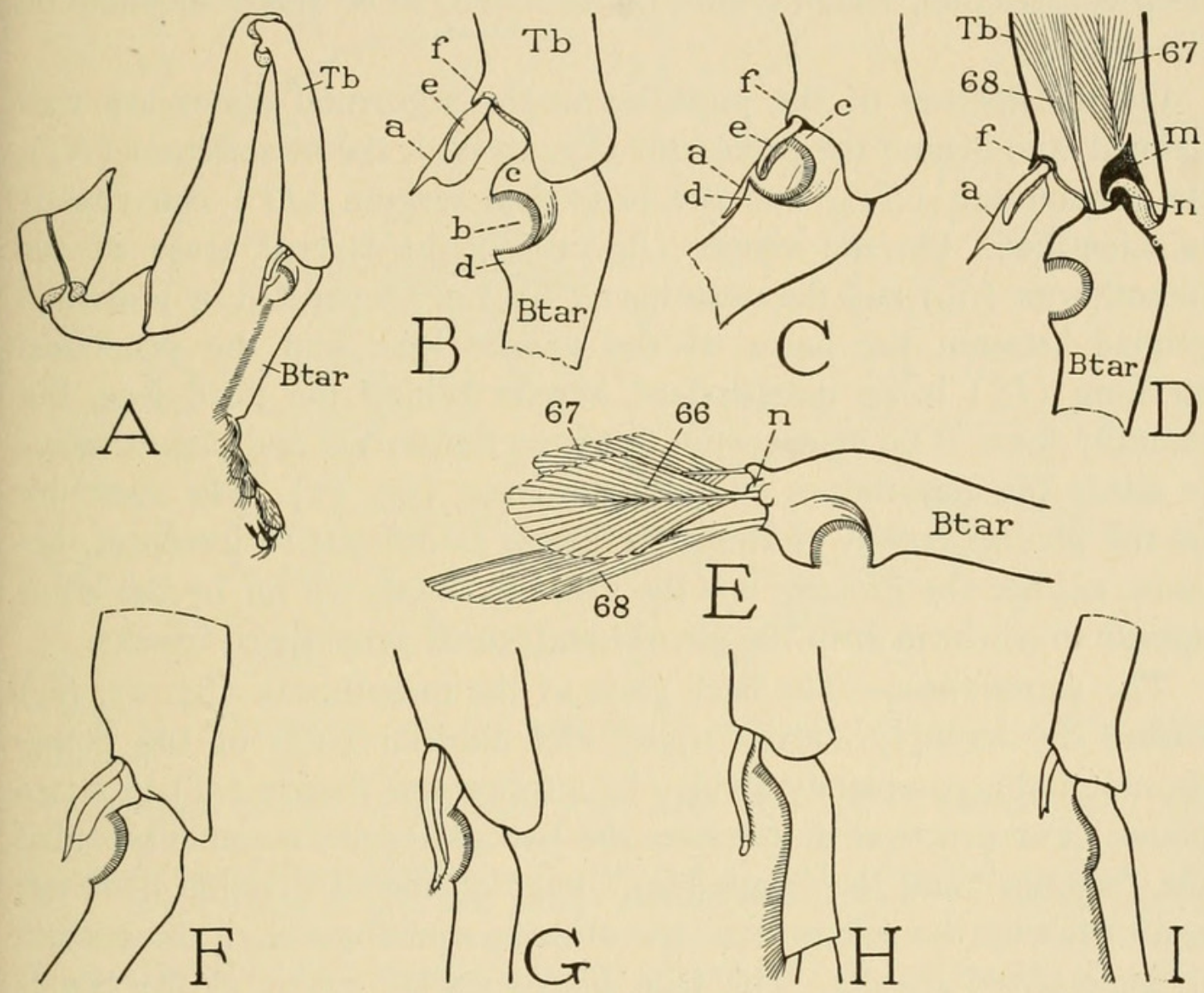

FIG. I4.-The antenna cleaner and the tibio-tarsal joint of the fore leg.

A, left fore leg of worker bee, showing antenna cleaner at base of first tarsomere (Btar). B, antenna cleaner open, anterior view. C, antenna cleaner closed. D, tibio-tarsal joint with muscles exposed by removal of anterior wall of tibia. E, base of first tarsomere with muscles, anterior view. F, antenna cleaner of Halictoides calochorti Ckll. G, same of Vespula maculata (L.). $\mathrm{H}$, same of Trogus vulpinus (Grav.). I, same of Orussus sayi Westw.

$a$, closing lobe (fibula) of antenna cleaner; $b$, tarsal notch of antenna cleaner; $c$, basal angle of notch; $d$, distal angle of notch; $e$, anterior lobule of closing lobe; $f$, stop-process of tibia; $m$, tibial articular process; $n$, tarsal articular process.

\section{THE PTEROTHORAX}

The wing-bearing section of the trunk in the clistogastrous Hymenoptera (fig. I5) is composed of the three body segments that are the mesothorax, the metathorax and the first abdominal segment of other insects. Though the notal plate of the prothorax $\left(N_{1}\right)$ is closely associated with the pterothorax, and looks like a collar on the 
front of the mesothorax, its connection with the latter is for its own security, since the pronotum serves principally for the attachment of prothoracic and head muscles, and takes no part in the pterothoracic mechanism. The pterothoracic segments, on the other hand, are intimately united to form a mechanical unit supporting the wings, the first and second legs, and the abdomen. Between the third pterothoracic segment, known as the propodeum, and the abdomen is a deep constriction, which allows the abdomen to be freely movable on the thorax.

On the dorsum of the pterothorax the segmental plates are well defined, and behind the pronotum (fig. I $5 N_{1}$ ), the mesonotum $\left(N_{2}\right)$, the metanotum $\left(N_{3}\right)$, and the propodeal tergum (IT) can readily be identified. On the venter (fig. I6 F) the sternal areas of the mesothorax $\left(S_{2}\right)$ and the metathorax $\left(S_{3}\right)$ are separated by a distinct groove between the bases of the middle legs, and the propodeal sternum $(I S)$ is an independent sclerite behind the third legs, but laterally there is no demarcation between the sternal and pleural areas in either the mesothorax or the metathorax (fig. I5). The structure of the pleural region of the pterothorax is difficult to interpret, because neither the grooves nor the areas distinctly set off by the latter appear to conform with the pleural pattern of generalized insects.

The mesonotum.-The back plate of the mesothorax (fig. I5, $N_{2}$ ) covers the strongly convex upper and anterior parts of the pterothorax, and is completely divided by a transverse fissure $(s f)$ into two plates. For practical description the two plates are commonly called the "scutum" and the "scutellum," but the line of division does not conform with the sulcus that separates the scutellum from the scutum in generalized insects. The true scutoscutellar sulcus in the bee is marked internally by a strong, arched notal ridge (fig. I6 D, NR), which on the middle of the back is coincident with the groove between the two mesonotal plates, but laterally follows a groove (fig. I5, ns) that curves posteriorly and sets off before it on each side an elongate oval or lenticular area ( $s c t$ ) which properly belongs to the scutum. The mesonotum of the bee and of related Hymenoptera, therefore, is secondarily divided into two major parts without regard to the original differentiation into scutum and scutellum by the external sulcus (ns) of the notal ridge. The true scutellum forms the prominent swelling on the highest part of the back $\left(\mathrm{Scl}_{2}\right)$ and tapers laterally to the posterior angles of the mesothoracic wing bases; the scutum $\left(\mathrm{Sct}_{2}\right.$ and $s c t$ ) covers the entire anterior rounded surface of the back between the notal sulcus ( $n s$ ) and the pronotum, and supports the greater 
part of the wing bases on its sides. In a study of the mechanism of the thorax rather than its morphology, however, it will be better to follow the mechanical pattern of the structure, in which the mesonotum consists of an anterior notal plate and a posterior notal plate. The reason for the division of the notum into these two plates will be clear when we study the wing mechanism.

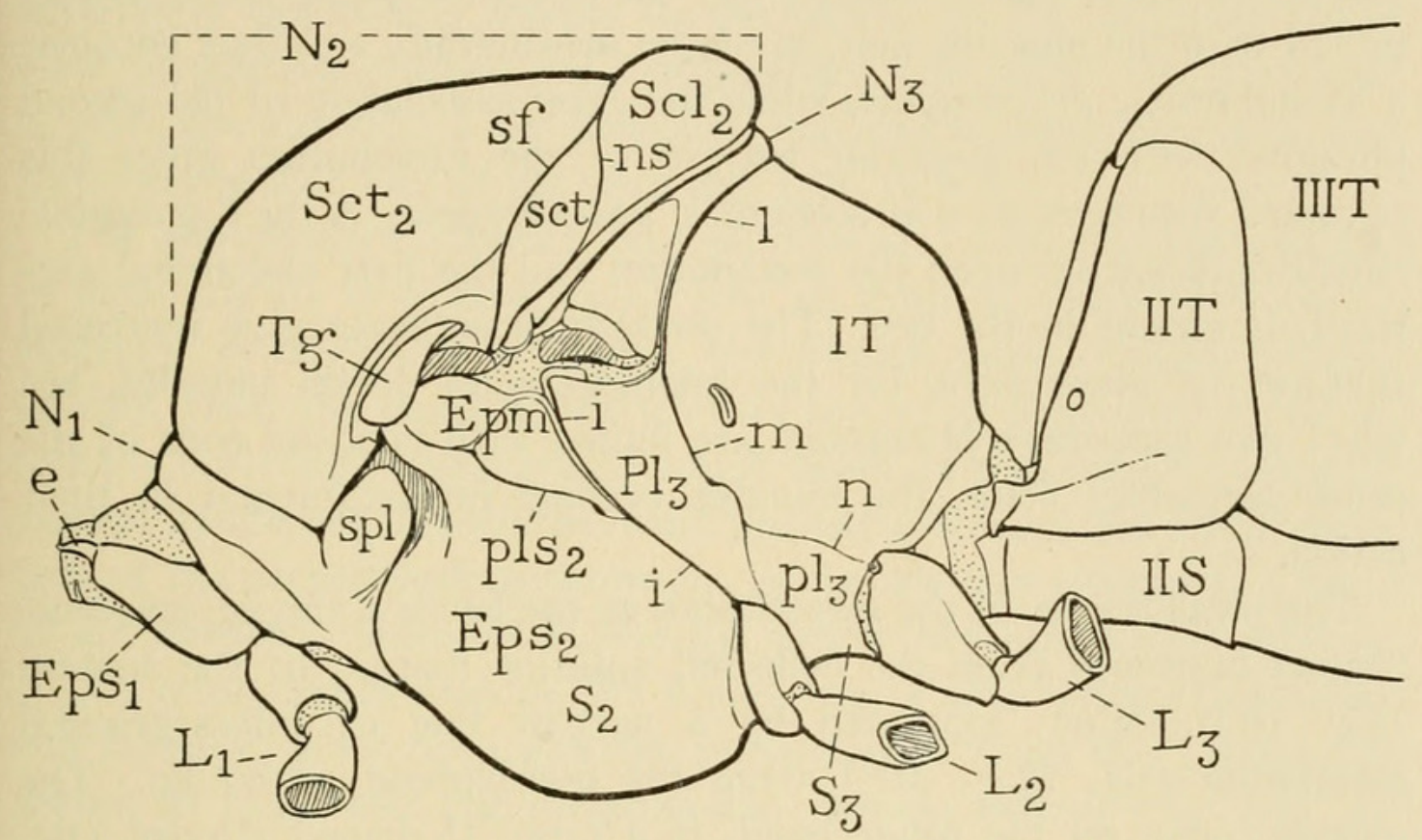

FIG. I5.-The thorax and the base of the abdomen (drone), left side, wings removed.

$e$, occipital process of proepisternum; Epm, epimeron of mesopleuron; $E p s_{1}$, prothoracic episternum; $E p s_{2}$, mesothoracic episternum; $i$, groove between mesopleuron and metapleuron; IIS, sternum of first abdominal segment; IIT $I I I T$, tergal plates of first and second abdominal segments; IT, tergal plate of propodeum; $l$, groove between metanotum and propodeum; $L_{1}, L_{2}, L_{3}$, legs; $m$, $n$, groove between metapleuron and propodeum; $N_{1}$, pronotum; $N_{2}$, mesonotum; $N_{3}$, metanotum; $n s$, primary notal sulcus between scutum and scutellum; $\mathrm{Pl}_{3}, \mathrm{pl} l_{3}$, upper and lower parts of metapleuron; $p l_{2}$, pleural sulcus of mesothorax; $S_{2}$, mesosternum; $S_{3}$, metasternum; $S c l_{2}$, scutellum of mesonotum; $\mathrm{Sct}_{2}$, scutum of mesonotum; sct, posterolateral subdivision of mesoscutum; $s f$, scutal fissure; $s p l$, spiracular lobe of pronotum; $T g$, tegula.

The anterior mesonotal plate (figs. I5, I6 C, $S c t$ ) is a large, shieldshaped sclerite (fig. I6 B) convex dorsally and strongly declivous anteriorly. Its front margin is deflected into the relatively small prephragma $(I P h)$, and on its lateral margins are the anterior notal wing processes $(A N P)$. The posterior, scutoscutellar mesonotal plate (figs. I5, I6 C, Scl, sct) is somewhat crescent-shaped as seen from above (fig. I6 A) or below (D) with the concave margin anterior; internally it is braced by the strong scutoscutellar notal ridge $(\mathrm{D}, N R)$. The lateral scutal areas of the plate bear the posterior notal wing processes, and the tapering ends of the scutellar 
area give attachment to the axillary cords of the wing bases. The posterior margin is deeply inflected laterally in the groove before the metanotum, and is here connected with the arms of the mesothoracic postphragma (fig. I6 C, D, G, 2Ph).

The phragmata of the insect thorax are primarily infoldings or ingrowths of the body wall on the intersegmental lines between the successive notal plates, but in the bee the first phragma is solidly united with the anterior margin of the mesonotum, and thus becomes a mesothoracic prephragma, while the close association of the second phragma with the posterior margin of the mesonotum gives this phragma the status of a mesothoracic postphragma. A third phragma, usually present between the metanotum and the first abdominal segment, is absent in the bee. The phragmata serve to give increased surfaces of attachment for the intersegmental dorsal muscles, but when two successive phragmata are united with opposite ends of the same segmental plate, the muscles become intrasegmental in their action.

The prephragma of the mesothorax of the bee is a simple, crescentshaped ingrowth from the deflected anterior margin of the scutum (fig. I6 B, IPh), separated by a narrow line of intersegmental membrane (fig. II F, $M b$ ) from the overlapping pronotum. The postphragma, on the other hand, is a large $\mathbf{U}$-shaped sclerite (fig. I6 $\mathrm{D}, 2 \mathrm{Ph}$ ) projecting far back under the metanotum into the cavity of the propodeum. Its only connections with the mesonotum are the attachments of its basal arms with the lateral ends of the scutellum. The base of each arm splits into an outer and an inner lamella $(G)$; the inner lamella $(v)$ is continuous with an infolding of the mesoscutellar margin, the outer lamella $(u)$ is reflected back into the anterior margin of the metanotum. The postphragma thus maintains its intersegmental relations, but there is in the bee no representative of the usual postscutellar supporting plate, or postnotum, other than the exposed parts of the inner lamellae of the phragma where the latter join the mesoscutellum.

The metanotum.-The back plate of the metathorax is a narrow transverse sclerite (fig. I6 E), constricted mesally and expanded laterally, closely interpolated between the mesonotum and the propodeum (fig. I5, $N_{3}$ ). The metanotum shows no division into scutum and scutellum, but its widened lateral parts form a prominent triangular area on each side. From the lateral margin of each triangular area there is deflected an irregular, semidetached sclerite (fig. 2I E, $n_{3}$ ), and before the latter is a smaller independent sclerite $(d)$, both of which sclerites give support to the wing base, as will 
be described later. The metanotum is separated from the propodeum by a prominent groove (fig. $15, l$ ), which forms a low ridge internally, but there is no metathoracic postphragma.

The propodeum.-The dorsal region of the propodeum (fig. I5, $I T$ ) is a large, strongly convex plate covering the posterior part of the pterothorax, containing the spiracles of its segment laterally, and posteriorly descending steeply to the petiole of the abdomen. This plate, commonly termed the "propodeum," is really the propodeal tergum, the sternum of the propodeal segment being a narrow ventral plate behind the bases of the third legs (fig. I6 F, IS).

The pterothoracic pleura.-The pleuron of a wing-bearing segment in most insects is differentiated into an anterior epistermum and a posterior epimeron by a groove, the pleural sulcus, which extends upward from the coxal articulation to the wing base, and forms internally a strong pleural ridge, which bears an apodemal process, or pleural apophysis, associated with the extremity of the endosternal arm of the corresponding sternum. In the bee this structural pattern of the pleuron is obscured in both the mesothorax and the metathorax.

The mesopleural area of the honey bee is well defined anteriorly and posteriorly by deep grooves separating it from the prothoracic notum in front and the metathoracic pleuron behind (fig. I5), its "free" upper margin borders the subalar membrane, ventrally, however, as already noted, the lateral sclerotization of the segment is continuous to the midline of the venter (fig. I6 F), and there is nothing to mark the transition from pleuron to sternum, except the ventral attachment of the huge dorsoventral muscle of the mesothorax (fig. I6 C, 72), which is assumed to arise on the sternum.

The upper part of the mesopleuron is crossed by an oblique groove (fig. I5, $p l s_{2}$ ), which appears to be the pleural sulcus, separating a small upper epimeral area $(E p m)$ from a large lower episternal area $(E p s)$. The groove contains near its posterior end a conspicuous pit (fig. I7 A, pla) that forms internally a small pleural apophysis (B, $P l A)$ intimately associated with the mesothoracic arm of the pterothoracic endosternum ( $\mathrm{G}, \mathrm{PlA}, S A_{2}$ ). Furthermore, the line of the posterior part of the groove is marked internally by a strong ridge $(\mathrm{B}, P l R)$ bearing the apophysis. These several features almost certainly identify the oblique groove $(\mathrm{A}, p l s)$ as the true pleural sulcus of the mesothorax. Beyond the apophysis the sulcus merges with the intersegmental groove (fig. I $_{5}, i$ ) that runs downward to the pleural articulation of the middle coxa. A second groove of the pleuron (fig. I7 $\mathrm{A}, e$ ) arises near the middle of the pleural sulcus, runs upward and posteriorly through the epimeral region, cutting the 

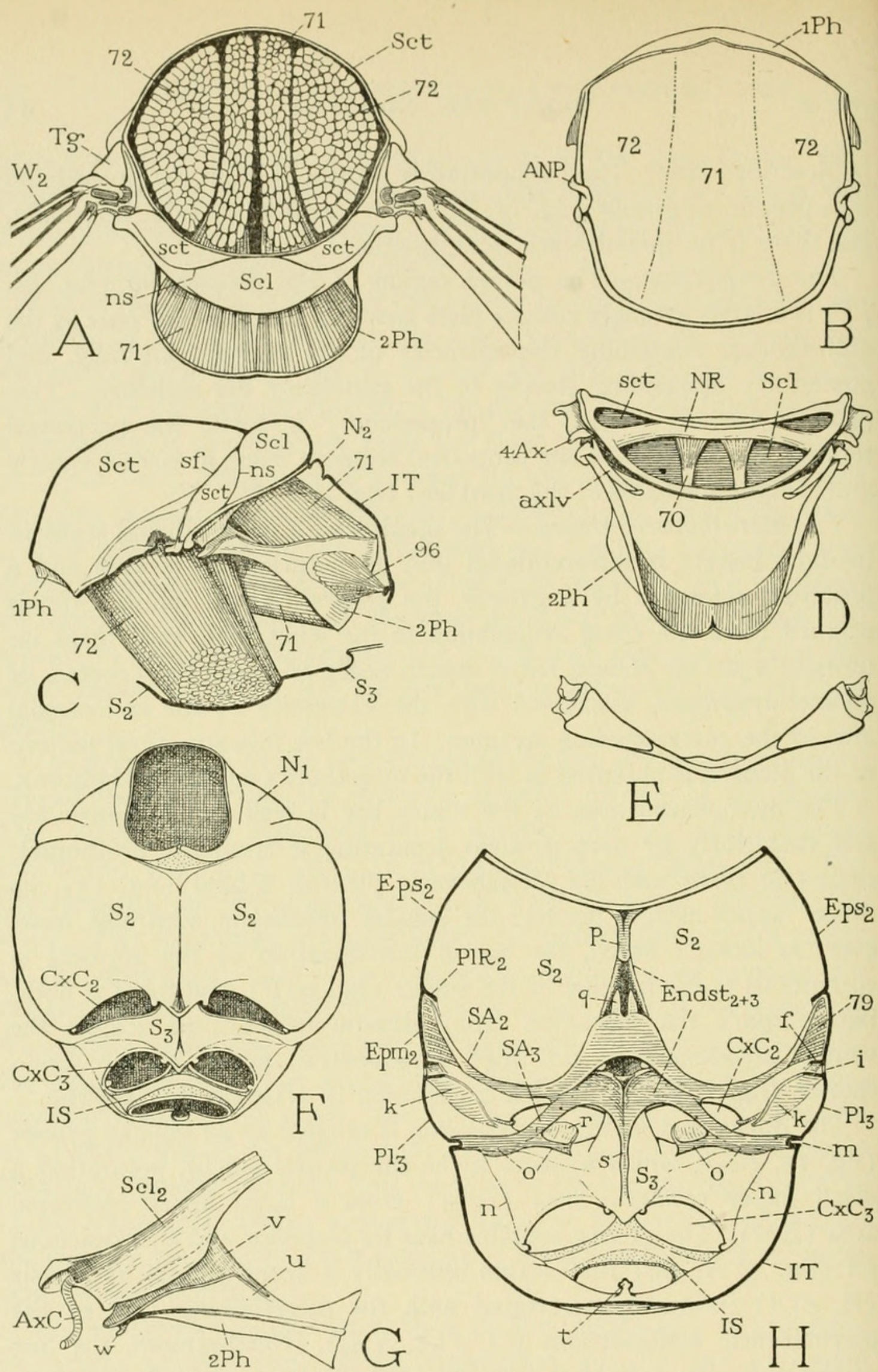

FIG. I6.-Dissection of the pterothorax (drone).

A, mesothorax and wing bases, dorsal view, with most of scutal area removed exposing ends of longitudinal and dorsoventral muscles. B, major scutal plate of mesothoracic notum, ventral view. C, pterothorax with pleural and propodeal walls removed on left side, showing phragmata and muscles. D, posterior notal plate of mesothorax and attached second phragma, ventral view. E, metanotum, dorsal view. F, ventral surface of thorax, legs and propectus removed. G, left lateral arm of mesoscutellum and corresponding stalk of second phragma, lateral view. $\mathrm{H}$, ventral half of pterothorax, dorsal view, showing endoskeletal structures.

$f$, posterior marginal ridge of mesopleuron; $i$, conjunctiva between mesopleuron and metapleuron; $k$, anterior inflection of metapleuron; $m$, groove between metapleuron and propodeum; pleuron; $k$, anterior ine of external groove $n$ (fig. 15 ); ns, notal sulcus; $o$, ridge of external groove $m$ (fig. 15 ); $p$, process of mesosternal ridge giving attachment to muscle 58 (fig. $22 \mathrm{C}$ ); $q$, process of supra$p$, process of mesosternal ridge giving attachment to muscle 52 (fig. $22 \mathrm{C}$ ); $r$, lobe of ridge $o$ giving attachment to basalar muscle of metathorax (fig. $2 \mathrm{IH}, I O I$ ); $s$, median ridge of metasternum: sf scutal fissure; $t$, median process of propodeum giving attachment to muscles o6 (C, and fig. $27 \mathrm{C}) ; u$, $v$, outer and inner lamellae of basal stalk of second phragma; $w$, articular process on stalk of second phragma for axillary lever. 
latter into two parts (Epm, epm), and then turns downward close to the posterior epimeral margin, and, along with the pleural sulcus, finally becomes lost in the intersegmental groove. This recurrent groove also forms a strong internal ridge $(\mathrm{B}, f)$, and it too has been regarded as the pleural sulcus (see Snodgrass, I925), but it has none of the distinctive features of the latter. Duncan (I939) gives the same interpretation of the pleural structure in Vespula as is given here for Apis. The obliteration of the lower part of the mesepimeron makes it appear that the middle coxa (fig. I5) has an intersegmental pleural articulation, though its ventral articulation is with the mesosternum (fig. I6 F).

Anteriorly the mesopleuron presents a deep concavity (fig. I7 A, $c$ ), which is covered by the spiracular lobe of the pronotum (fig. I 5 $s p l)$; its dorsal margin forms a long elevation, on the anterior part of which (fig. I7 A, $d$ ) articulates the basalar sclerite (fig. I9 B, $B a$ ), and on the posterior part (WP) the second axillary of the wing base $(2 A x)$. Behind the wing process $(W P)$ the descending margin of the epimeron is inflected mesally in a wide plate (fig. I7 B, Epm') supported on the ridge $(f)$ of the recurrent suture. The inner edge of this inflected plate carries an elongate sclerite $(\mathrm{G}, h)$, attached posteriorly to the base of the postphragma (fig. I9 I), which supports the small subalare $(S a)$.

The inner surface of the mesopleuron presents an unusual structure (fig. I7 B). From its anterior and upper margin, between the first spiracle and the wing process, there is reflected posteriorly a large plate $(g)$, the lower part of which is bridged to both the pleural ridge $(P l R)$ and the recurrent ridge $(f)$ by a strong arch. Between this plate and the outer pleural wall is a flat pocket open above and below the bridge (as indicated in the figure by an arrow), which lodges one of the muscles of the third axillary sclerite of the wing base $(\mathrm{E}, 76 a)$. Behind the upper part of the pocket is seen the inflected external plate of the epimeron $\left(B, E p m^{\prime}\right)$ united with the recurrent ridge $(f)$. The thick pleural ridge $(P l R)$ ends posteriorly in the small pleural apophysis $(P l A)$.

The metathoracic pleuron is a narrow, oblique plate on the side of the pterothorax (fig. I5), extending from the wing base above to the hind coxa below, between the mesopleuron and the propodeum. It is divided by a groove into a larger upper part $\left(\mathrm{Pl}_{3}\right)$ and a smaller lower part $\left(p l_{3}\right)$, but the latter is continuous ventrally with the metasternum $\left(S_{3}\right)$. The dorsal plate (fig. 2I C, $P l_{3}$ ) supports on its upper margin the basalare $(B a)$, the second axillary $(2 A x)$, and the small subalare $\mathrm{S}(\mathrm{S})$ of the metathorax; it includes, therefore, in its upper part at 
least, both the episternum and the epimeron, though a separating sulcus is not present. The anterior pleural margin is inflected in a high apodemal ridge (figs. $\mathrm{I} 6 \mathrm{H}, 2 \mathrm{I} \mathrm{C}, k$ ); posteriorly the upper pleural plate is limited by a deep groove (fig. ${ }^{5}, m$ ) separating it from the propodeum, which forms a strong internal ridge (fig. I6 $\mathrm{H}$, $o$ ) with which the endosternal arm of the metathorax $\left(S A_{3}\right)$ is fused. From the lower part of the groove $m$ (fig. I5) a weak impression ( $n$ ) goes posteriorly to the coxal articulation. It is evident, therefore, that the epimeron of the metathorax, as that of the mesothorax, has been mostly eliminated, and that the apparent intersegmental groove $(m, n)$ before the propodeum includes the lower part of the pleural sulcus extending to the coxal articulation. The position of the posterior pleural muscles behind this groove and its internal ridge confirms the above interpretation.

The thoracic spiracles.-Of the three pairs of spiracles on the thorax those of the first pair are of particular interest because of their size, position, and structure. They are contained in the membranous inner walls of the spiracular lobes of the pronotum (fig. I7 C, $I S p)$, and therefore open toward the body into the depressions of the mesopleura $(\mathrm{A}, c)$ beneath the lobes, the entrances to which are guarded by dense fringes of plumose hairs on the lobe margins. The reflected lower margin of each pronotal lobe is supported on a small, rectangular cartilagelike plate $(\mathrm{D}, a)$ arising from a lobe of the anterior margin of the mesopleuron $(\mathrm{A}, \mathrm{B}, b)$. The plate is a thickening of the intersegmental membrane, and serves to prevent movement of the spiracular lobe. The orifice of each spiracle $(\mathrm{C}, \mathrm{F})$ is a simple opening in the spiracular membrane leading into a large tracheal trunk (Tra) going forward into the prothorax. Above the aperture is an oval plate $(\mathrm{F}, O p)$, the ventral edge of which forms the upper lip of the aperture; the lower lip is merely the soft edge of the surrounding membrane. The plate can be pulled down over the aperture by a slender muscle (73) and thus serves as a closing lid, or operculum, of the spiracle. The occlusor muscle arises from a small knob on the anterior margin of the mesopleuron ( C, 73), and is inserted by a tendon in the membrane close to the anterior margin of the spiracular orifice (F), not directly on the operculum. The simple closing apparatus of this spiracle would appear to be more effective in blocking the entrance of air into the trachea than for preventing its egress, but the soft lower lip of the closed aperture is easily pushed inward, and probably gives little resistance to parasitic mites seeking an entrance. 
The second spiracles lie below the wings in the membrane concealed between the upper edges of the mesothoracic and metathoracic pleura. They are minute apertures without a closing apparatus, and have been
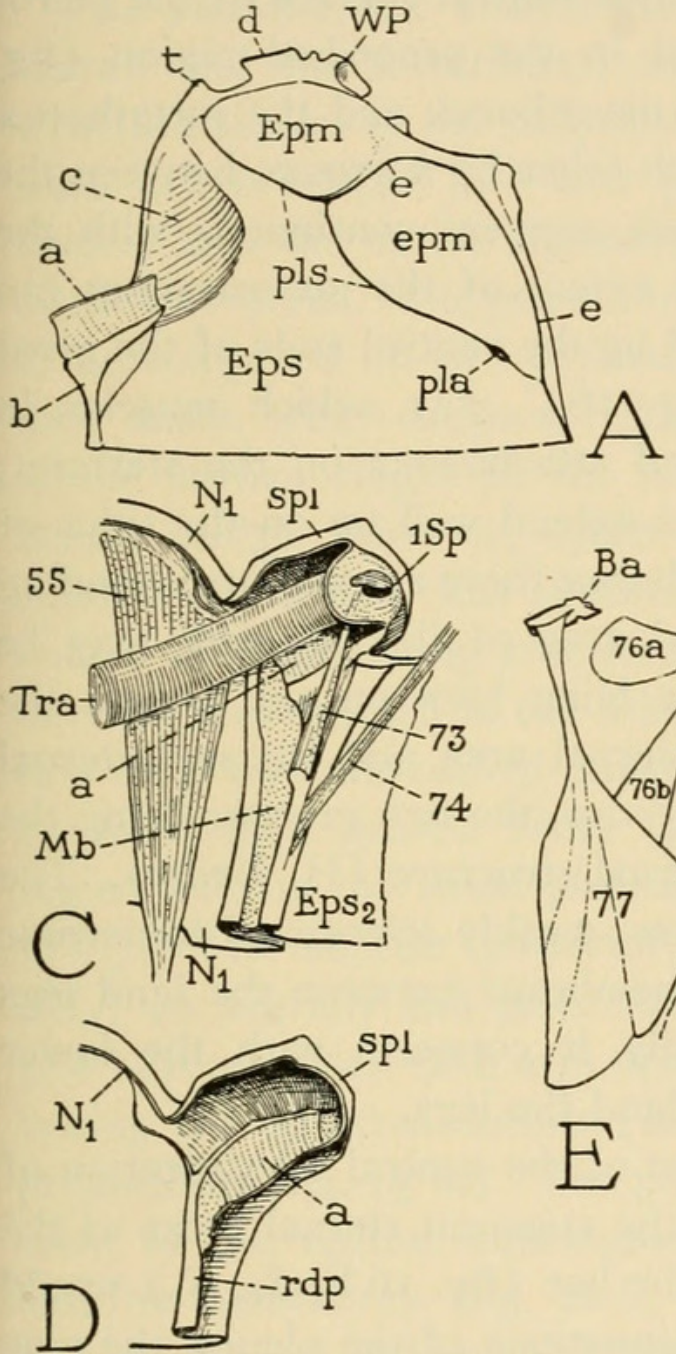

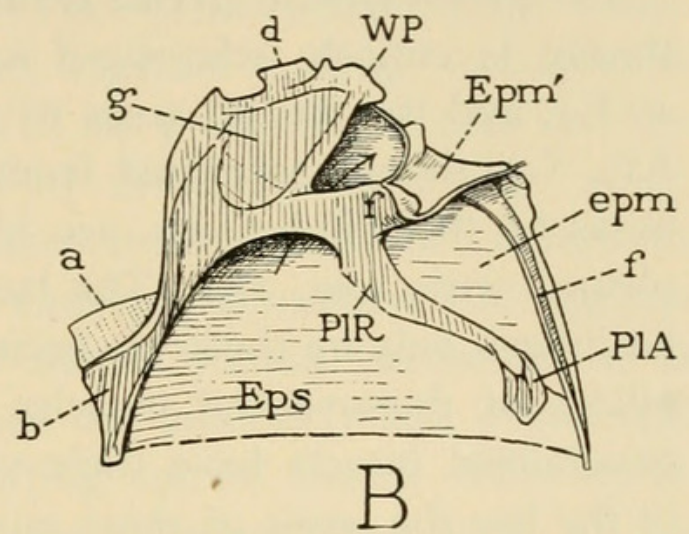

FIG. I7.-The mesopleuron and the first spiracle (drone).

A, left mesopleuron, lateral view. B, right mesopleuron, mesal view. C, right first spiracle and adjoining parts of prothorax and mesothorax, mesal view. $\mathrm{D}$, right spiracular lobe of pronotum, mesal view. E, mesopleural muscles arising on episternum, right side, mesal view. F, right first spiracle and connected trachea, mesal view. G, posterior pleural muscles of mesothorax, right side, mesal view.

$a$, platelike thickening of intersegmental membrane connecting spiracle lobe of pronotum with mesepisternum; $b$, lobe of mesepisternum supporting $a$; $c$, depression of mesepisternum covered by spiracular lobe of pronotum; $d$, lobe of mesepisternum supporting basalare; $e$, recurrent groove of mesopleuron; $f$, internal ridge of recurrent groove; $g$, mesal inflection of mesopleuron continuous with pleural ridge $(P l R) ; h$, epimeral plate supporting subalare; $t$, point at which mesoscutum is supported on pleuron.

observed by the writer only in the worker. The third spiracles are those of the propodeum, and have the structure of the other abdominal spiracles. Each is provided with a short occlusor muscle (fig. $27 \mathrm{~B}, 122$ ) stretched between the two ends of the spiracle, and 
with a long slender dilator muscle ( 123 ) arising ventrally from a small knob on the pleural margin of the metacoxal cavity just before the pleural coxal process $\left(C x P_{3}\right)$.

The pterothoracic sternal areas.-The ventral surface of the pterothorax is entirely sclerotized except in the propodeal region (fig. I6 F), and the sternal areas of the mesothorax and the metathorax $\left(S_{2}, S_{3}\right)$, though separated from each other by a groove between the bases of the middle legs, are in each segment continuous with the pleural areas (fig. I5). The lateral extent of the mesosternum can be judged only by the areas occupied by the ventral ends of the great pillars of dorsoventral muscles (fig. I6 C, 72), which muscles in generalized insects have their ventral attachments on the sternum; in the bee the bases of these muscles extend well up on the sides of the pleurosternal areas. In the metathorax there are no corresponding tergosternal muscles, but the sternal area of this segment may be regarded as that part of the venter lying between and before the hind legs $\left(\mathrm{F}, S_{3}\right)$. Both the mesosternal area and the metasternal area are marked each by a median groove, the two grooves being the lines of inflection of a large endosternal structure $(\mathrm{H}$, Endst). The sternum of the propodeum is a narrow, weakly sclerotized transverse band $(F, I S)$ lying in the ventral membrane between the hind legs and the abdominal petiole. Laterally it connects with the lower margins of the propodeal tergum behind the legs.

According to a recent interpretation of the ventral sclerotization of the insect thorax by Ferris (I940), the apparent sternal areas of the mesothorax and the metathorax of the bee (fig. I6 F, $S_{2}, S_{3}$ ) would probably be regarded as ventral continuations of the pleura, the true sternal parts being supposed to be entirely folded in to make the base of the endosternum. The evidence presented in favor of this interpretation, however, is argumentative, and does not include a consideration of the dorsoventral muscles, which appear to be tergosternal in their attachments among generalized insects. It also seems to assume that morphological regions of a segment necessarily follow shifts in the sclerotization, rather than the converse, that sclerotized areas may expand or contract in a continuous and otherwise stationary integument. In the chalastogastrous family Xyelidae, among the Hymenoptera, the episternal sclerotizations of the mesothorax are extended downward onto the ventral surface, where they are continuous across the median line in a narrow or wide bridge. Behind the bridge, however, is a large, triangular basisternal plate with a strong median groove, and on this sternal plate are attached the lower ends of the dorsoventral muscles. In most other Hymenoptera the 
muscle-bearing areas of the ventral sclerotization are continuous with the episternal areas, as in the honey bee, but in some forms, as in the tenthredinoid Arge, the two are separated by distinct sutural lines.

The pterothoracic endosternum.-The sternal areas of the mesothorax and the metathorax support together a large endoskeletal structure similar to the endosternum of the prothorax, except that the sides are produced into two pairs of divergent arms (fig. $16 \mathrm{H}$, Endst) and the entire structure arises from the median inflections of the two sterna. The pterothoracic endosternuum, therefore, appears to be composed of two ordinary $\mathbf{Y}$-shaped sternal furcae united at their bases, which latter are stretched out anteriorly and posteriorly in the median ridges of the supporting sternal plates. The supraneural bridge, however, spans the space between the bases of the anterior arms, and thus appears to belong to the mesothoracic component only. The long anterior, or mesothoracic, arms $\left(S A_{2}\right)$ extend laterally and upward to the sides of the thorax, where each arm ends in a thin, forward-curved expansion that gives attachment to a large group of short muscle fibers (79) arising on the posterior epimeral region and on the small pleural apophysis of the mesopleuron (fig. I7 G). The shorter posterior, or metathoracic, arms (fig. I6 $\mathrm{H}$, $S A_{3}$ ) go laterally and posteriorly over the bases of the second legs, just beyond which each arm unites solidly with a thick ridge $(o)$ following the external suture $(m)$ between the metapleuron and the propodeum (fig. I $5 \mathrm{~m}$ ). The pterothoracic endosternum gives attachment to the ventral intersegmental muscles between the prothorax and the mesothorax (fig. $22 \mathrm{C}, 5^{2}, 5^{8}$ ), and between the metathorax and the second abdominal segment (fig. $27 \mathrm{E}, I I 8, I I 9$ ), to muscles of the second and third legs (figs. $22 \mathrm{C}, 24 \mathrm{~A}$ ), to the muscles of the axillary levers of the mesothoracic wings, and to the tergosternal muscles of the metathorax (fig. 2I F, 97, 98, 99).

\section{THE WINGS}

The movements of an insect's wings fall into two distinct categories : first, there are the movements of flight when the wings are extended, and second, the movements by which the wings are turned posteriorly over the body when at rest, or again brought into the extended position preliminary to flight. The action of the wing muscles that produce the movements of flight depends chiefly on structural adaptations in the skeletal parts of the thorax; the effect of the muscles concerned in the flexion and extension of the wings, on the other hand, is mainly dependent on structural details in the wing bases. 
In other words, both sets of wing movements are produced by primitive muscles that have become motors of the wings by modifications in the skeletal elements, the primary changes that made flight possible being mostly in the thoracic skeleton, the secondary adaptations for flexion and extension being largely in the bases of the wings themselves.

In the bee the principal muscles of flight are the huge longitudinal and dorsoventral muscles of the mesothorax (fig. I6 C). For this

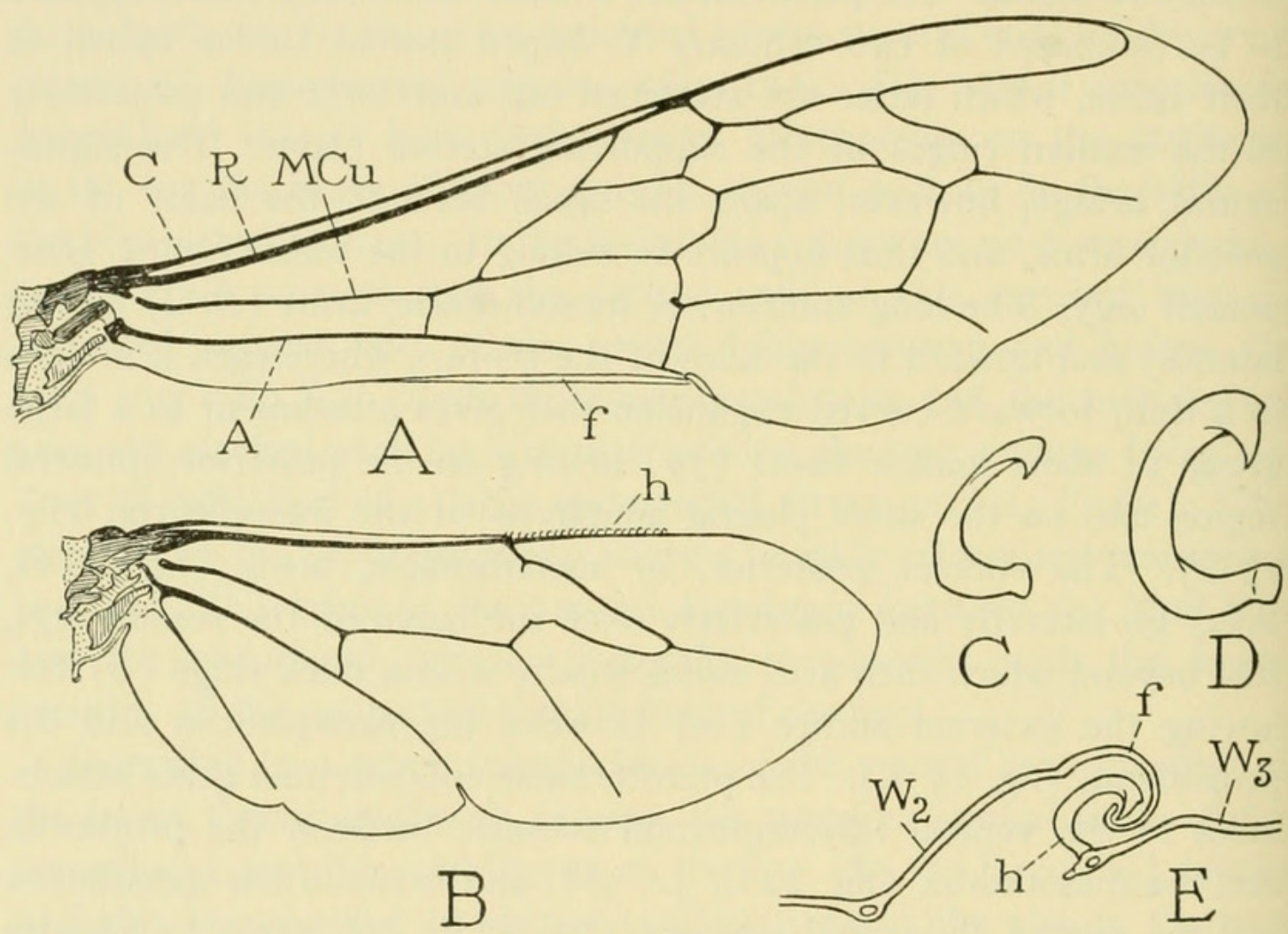

FIG. I8.-The wings.

A, B, fore and hind wing of drone. C, hook of hind wing of worker. D, hook of hind wing of drone. E, section of interlocked wing margins.

$f$, deflected fold on posterior margin of fore wing; $h$, hooks on anterior margin of hind wing.

reason probably the two wings on each side, when extended, are attached to each other. The coupling mechanism consists of a series of small hooks on the anterior margin of the hind wing (fig. I $8 \mathrm{~B}, h$ ) and a fold on the posterior margin of the fore wing $(\mathrm{A}, f)$. The hind-wing hooks, or hamuli (C, D), curve upward and backward from the marginal vein of the wing with a twist in the direction of the wing tip. The fold of the fore wing is a rounded deflection of the posterior part of the elevated wing area behind the last vein, and has a narrowly upturned margin $(\mathrm{E}, f)$. When the wings are extended preparatory to flight the fore wing is drawn over the upper 
surface of the hind wing, and the hooks of the latter become engaged in the fold of the former (E).

The fore wing.-The mesothoracic wing of the bee is much larger than the hind wing (fig. I8) and has a stronger and more elaborate venation. The four veins that arise at the base of the wing are probably costa $(\mathrm{A}, C)$, radius $(R)$, media-cubitus $(M C u)$, and an anal, or vannal, vein $(A)$. It is difficult to identify the branches of these main veins in the coarse reticulation of the distal part of the wing, but it will not be necessary for a study of the wing mechanism; an understanding of the basal structure of the wing, however, is of much importance.

The base of the fore wing is overlapped by a large scalelike lobe known as the tegula (figs. I5, I9 A, $T g$ ), which is flexibly attached to the margin of the scutum just before the wing. In the wasp Vespula Duncan (I939) finds a small depressor muscle of the tegula arising on the scutum, but the writer has observed no muscle connected with the tegula in Apis.

In the naturally extended position of the fore wing, the wing base is rolled on itself (fig. I9 A), the posterior part being turned downward so that little of it is visible when viewed from above. If the basal region is artificially spread out flat (D) the posterior part is seen to be an extensive area widening toward its attachment on the body. The whole region of the wing base presents at first sight a confusion of small irregular parts, some of which are expansions of the wing veins and others independent sclerites in the basal wing membrane. A closer study, however, soon shows that there is an orderly arrangement of the parts and a mechanical interrelationship between them, which latter becomes more evident when the wing is moved.

The anterior part, or humeral area, of the wing base (fig. I9 D) is occupied by a large irregular sclerotization, here termed the humeral complex because it appears to include parts derived from the bases of the first three veins and also a remnant of the subcosta, which is suppressed in the distal part of the wing. The posterior part of the wing base, or axillary area, contains several discrete sclerites, which are the first, second, third, and fourth axillaries (IAx, 2Ax, $3 A x$, $4 A x)$, and a prominent, transversely elongate median plate $(m)$. All these sclerotizations are held in a basal wing membrane, bordered posteriorly by a corrugated marginal thickening known as the axillary $\operatorname{cord}(A x C)$, which is continuous on the one hand with the posterior margin of the wing, and on the other with the lateral extremity of the scutellum of the mesonotum $(\mathrm{F}, \mathrm{J}, A x C)$. 
The first axillary (fig. I9 E, $I A x$ ) is an elongate sclerite with a large anterior head $(b)$ turned laterally on a curved neck, posterior to which is a wide lateral projection $(f)$ and a smaller articular lobe (c) on the mesal margin. The sclerite is attached to the side of the mesonotum in such a manner $(\mathrm{J})$ that its neck rests on the anterior lobe $(p)$ of the notal wing process $(\mathrm{F})$, and its articular lobe is closely hinged to the posterior lobe $(q)$ of the wing process, while the tapering posterior end rests and turns in a concave surface on the lateral margin $(r)$ of the scutal area of the mesonotum (J. sct), where normally (D) it is concealed in a pocket of the wing membrane beneath the fourth axillary $(4 A x)$. In the natural position of the extended wing (A) the first axillary $(I A x)$ stands almost vertical; in the flattened wing base (D) it is seen that its head abuts against the humeral complex, and the lateral projection underlaps the median plate $(m)$. The first axillary is the anterior hinge plate of the wing.

The second axillary (fig. I9 E, $2 A x$ ) is a thick sclerite standing vertically in the wing base. Its exposed upper surface $(i)$ appears as a small oval plate (D) lying in the notch between the head and the body of the first axillary. Ventrally the second axillary articulates by an anterior knob $(\mathrm{E}, \mathrm{B}, j)$ with the wing process of the pleuron, and by a posterior arm $(k)$ is associated with the subalar sclerite (B, $\mathrm{Sa}$ ) on the upper edge of the pleuron. The second axillary is the pivotal sclerite of the wing base, since it is the only axillary sclerite that articulates with the pleuron and gives the wing a solid support from below. In the bee the second axillary has no direct connection with any of the wing veins, though in most insects it is connected with the base of the radius.

The third axillary (fig. I9 E, $3 A x$ ) is an elongate sclerite lying close along the axillary cord (D), with its tapering distal end associated with the enlarged base of the single anal vein of the wing. Proximally the third axillary bears a large lobe $(\mathrm{E}, l)$ projecting anterior to its axis, on which are inserted three muscles arising on the mesopleuron $(\mathrm{H})$. The small, flat uppermost muscle $(\mathrm{H}, 76 a)$ arises in the pocket of the inner wall of the pleuron (fig. I7 B) beneath the reflected plate $(g)$; the other two larger muscles (fig. I9 $\mathrm{H}$, $76 b, 76 c)$ arise ventrally on the episternum. The third axillary is the only axillary sclerite of the wing base provided with muscles; it is the effective skeletal element of the wing-flexing mechanism, and may be termed the flexor sclerite.

The fourth axillary (fig. I9 E, $4 A x$ ) is a small, irregularly triangular sclerite lying transversely in the posterior angle of the wing base (D), articulated mesally on the lateral margin of the scutal area 

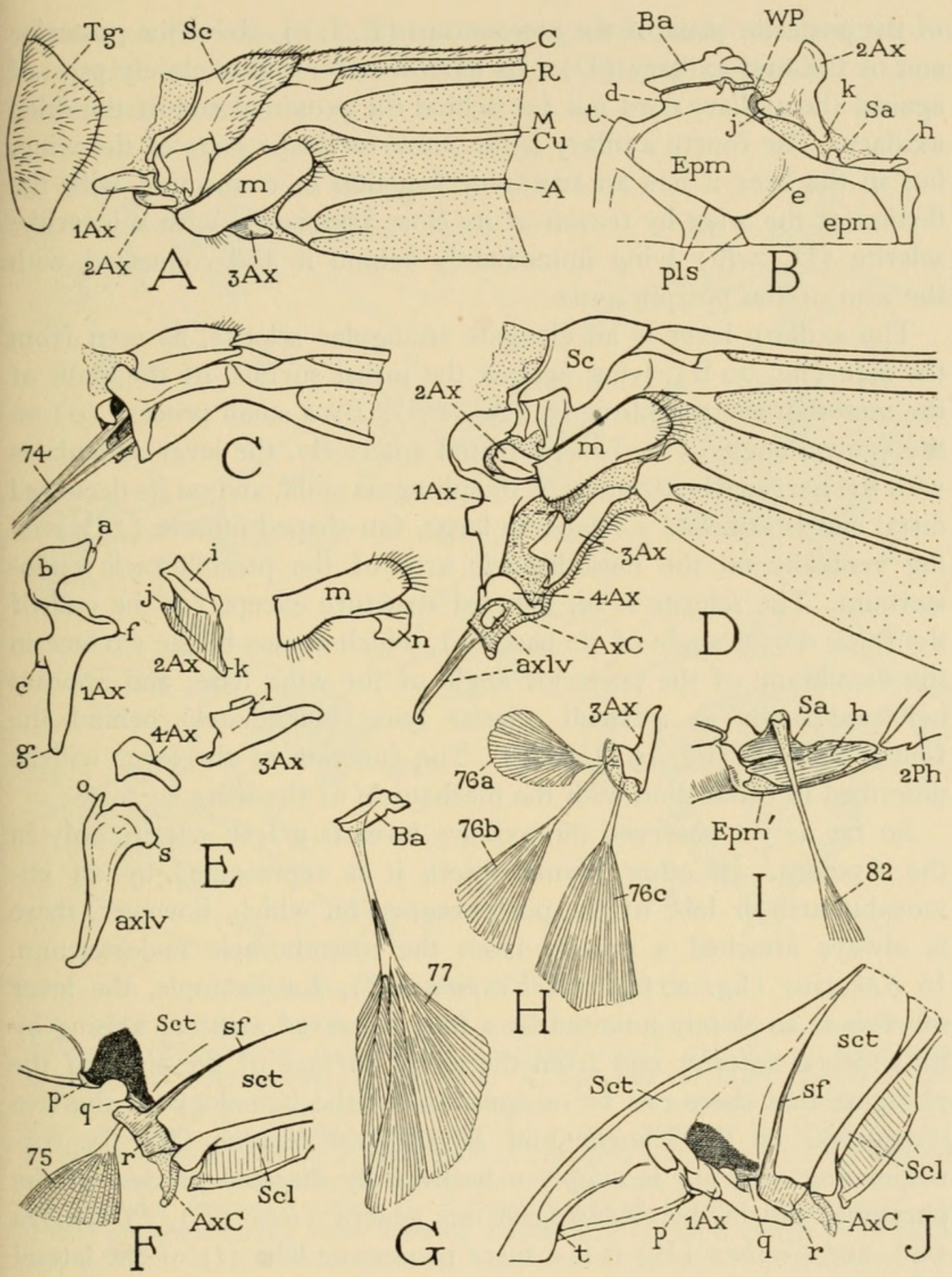

FIG. I9.-Details of the mesothoracic wing base, its articulations, and pleural muscles (drone).

A, tegula $(T g)$, and wing base in natural position of extended wing. B, upper part of mesopleuron, showing pleural articulation of basalare and second axillary. $\mathrm{C}$, humeral area of wing base, ventral view. D, base of right wing with axillary region flattened, dorsal view. $\mathrm{E}$, axillaries and axillary lever of right wing. F, left margin of mesonotum in region of wing attachment. G, right basalare and its muscle, mesal view. H, right third axillary and its muscles, mesal view. I, right subalare and its muscle, with supporting sclerite $(h)$ on edge of epimeron, mesal view. J, left lateral part of mesonotum, with first axillary in natural position.

$a$, articular surface of first axillary with humeral complex of wing base; $b$, head of first axillary; $c$, notal hinge-lobe of first axillary; $d$, lobe of mesopleuron supporting basalare; $e$, recurrent suture of mesopleuron; $f$, lateral process of first axillary; $g$, posterior end of first axillary; $h$, supporting plate of subalare; $i$, exposed dorsal surface of second axillary; $j$, pleural articular process of second axillary; $k$, posterior ventral process of second axillary; $l$, muscle lobe of third axillary; $m$, median plate of wing base; $n$, distal lobe of median plate; $o$, articular process of axillary lever; $p$, anterior lobe of anterior notal wing process supporting neck of first axillary; $q$, posterior lobe of anterior notal wing process articulating with hingelobe of first axillary $(c) ; r$, posterior notal wing process; $s$, exposed head of axillary lever; $t$, point at which mesoscutum is supported on anterior angle of pleuron. 
of the posterior plate of the mesonotum $(\mathrm{F}, \mathrm{J}, r)$, above the posterior end of the first axillary (D). Its narrow outer end is closely pressed against the axillary cord not far behind the proximal end of the third axillary. The fourth axillary is the posterior hinge plate of the wing, but in the bees it has an accessory function in connection with the flexion of the wing by reason of its close association with a leverlike sclerite (D, axlv) lying immediately behind it and connected with the arm of the postphragma.

The axillary lever is an elongate triangular sclerite, as seen from the side (fig. $20 \mathrm{~B}$ ), lying against the mesal surface of the stalk of the mesothoracic postphragma (A, axlv). By a small process $(o)$ on the ventral angle of its base, directed anteriorly, the lever articulates with the narrowed extremity of the phragma stalk, and on its decurved distal end is attached a relatively large, fan-shaped muscle $(78)$ arising ventrally on the mesothoracic arm of the pterothoracic endosternum. The sclerite is an internal structure except for the end of the thick dorsal angle of its base $(s)$, which comes to the surface in the membrane of the posterior angle of the wing base, and appears here externally as a small sclerite lying immediately behind the fourth axillary (fig. I9 D, axlv). The function of the lever will be described in connection with the mechanism of the wing.

So far as yet observed the axillary lever is a free sclerite only in the Apoidea. In other Hymenoptera it is represented by an immovable arm or lobe of the postphragma, on which, however, there is always attached a muscle from the mesothoracic endosternum. In Sphecius (fig. 2o C) and Vespula (D), for example, the lever of Apis is so closely imitated by a long decurved arm $(t)$ arising by its widened anterior end from the mesal surface of the stalk of the phragma that there can be no question of the homology of the two structures. In the ichneumonid Megarhyssa lunator (E) the corresponding arm $(t)$ is simply a backwardly directed process of the phragma, and in the chalastogastrous genera Sirex (F), Pteronidea $(\mathrm{G})$, and Cimbex $(\mathrm{H})$ it is a mere process or lobe $(t)$ of the lateral part of the phragma giving attachment to an endosternal muscle $(78)$. In these last forms the muscles evidently pull downward on the phragma and probably depress the phragma-bearing postnotal plate $\left(\mathrm{PN}_{2}\right)$. According to Duncan (1939) the muscle of the phragma arm in Vespula "resists the tendency of the indirect depressor muscle of the forewing (attached on the phragma) to lift the mesopostphragma, and helps to retract the phragma to the position of rest following a contraction of the indirect depressor muscle." The development of this structure into an accessory of the wing-flexing ap- 
paratus in the bees is a highly interesting example of the evolution of a special mechanism from a structure having originally a quite different function.

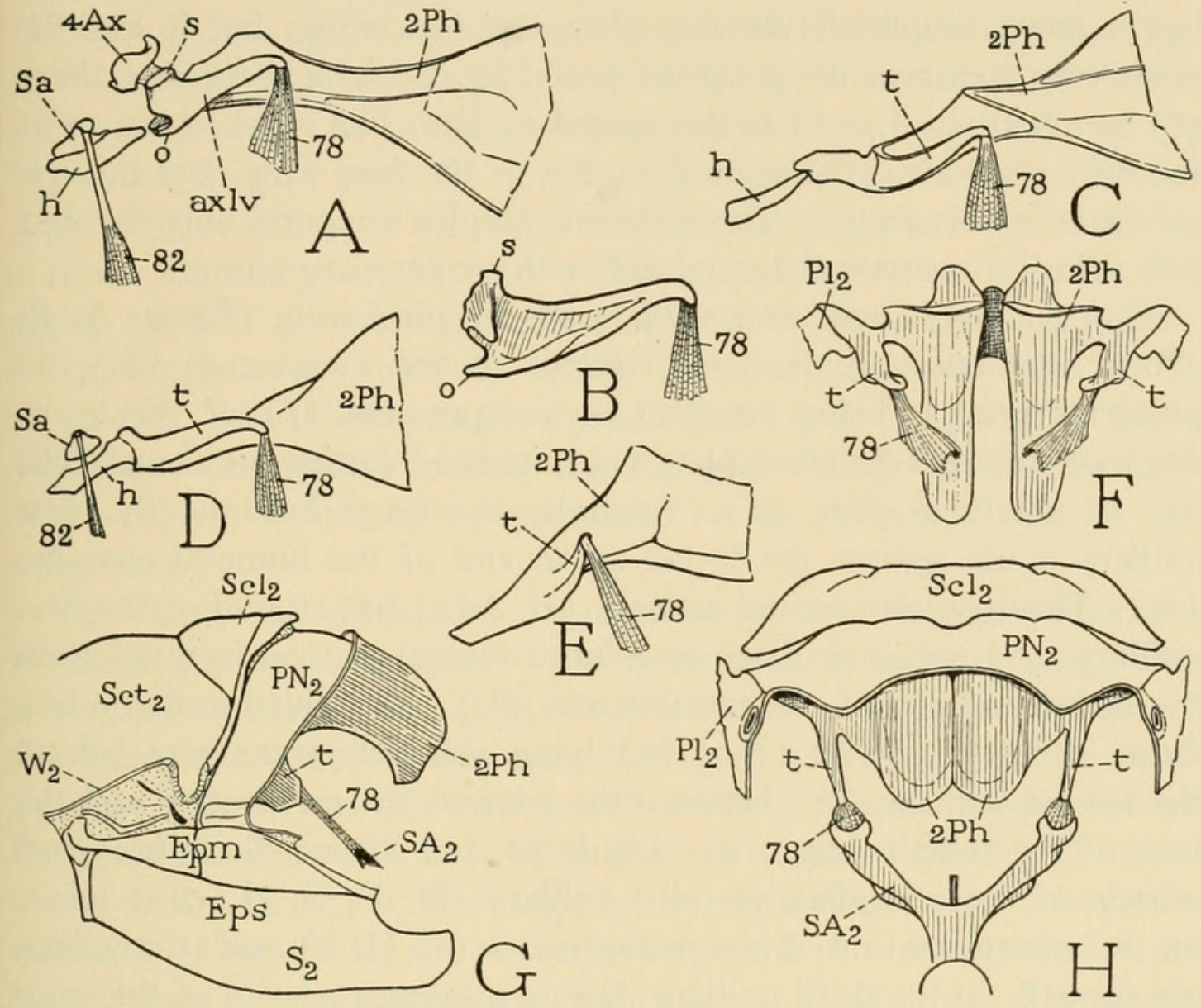

FIG. 20.-Evolution of the axillary lever.

A, Apis mellifera L., drone, right stalk of second phragma, mesal view, showing axillary lever ( $a x l v)$ and associated fourth axillary of wing base. B, same, left axillary lever, lateral view. C, Sphecius speciosus (Drury), right stalk of second phragma with mesal process $(t)$ corresponding with axillary lever of Apis. D, Vespula maculata (L.), same parts as in Sphecius (C). $\mathrm{E}$, Megarhyssa lunator (F.), right stalk of phragma with simple muscle process, mesal view. F, Sirex abbottii Kirby, second phragma and lateral muscle lobes $(t)$, anterior (ventral) view. G, Pteronidea ribesii (Scop.), mesothorax and second phragma, lateral view, showing muscle lobe $(t)$ with muscle from furcal $\operatorname{arm}\left(S A_{2}\right)$. H, Cimbex americana Leach, posterior end of mesonotum with second phragma, showing lateral muscle processes $(t)$ connected by short muscles $(78)$ with arms of mesosternal furca.

$h$, supporting sclerite of subalare; $o$, articular process of axillary lever; $s$, exposed head of axillary lever associated with fourth axillary sclerite; $t$, muscle-bearing process of second phragma of non-apoid Hymenoptera corresponding with axillary lever (axlv) of Apoidea.

The large, transversely elongate median plate in the base of the fore wing (fig. I9 $\mathrm{E}, \mathrm{m}$ ) lies close behind the humeral complex (A, D), and forms a link between the first and second axillaries at its mesal end, and the enlarged base of the fourth wing vein at its 
distal end. In most insects the median plate is associated with the bases of the veins media and cubitus, but in the Hymenoptera these two veins appear to be combined, and united basally with the radius.

The hind wing.-The small metathoracic wing of the bee (fig. I $8 \mathrm{~B}$ ) has a more simplified venation than the fore wing, but it has the same vein stems in its proximal part (fig. 2I A) except that there are two veins $(I A, 2 A)$ in the vannal region. The basal structure of the hind wing also is simpler than that of the fore wing, and there is no metathoracic tegula. The humeral complex supports only the first two veins; a median plate and a fourth axillary are absent.

The relatively small first axillary of the hind wing (fig. 2I A, B, $I A x)$ articulates on the lateral plates of the metanotum (E), its posterior process being received in a depression $(f)$ of the lower angle of the semi-detached plate $\left(n_{3}\right)$, its head resting on a small lobe $(e)$ of the free plate $(d)$. Laterally the large head of the first axillary abuts against the broad mesal end of the humeral complex (A). The irregular second axillary $(\mathrm{B}, 2 A x)$ lies laterad of the first axillary (A) and articulates by a large ventral process in a notch on the dorsal margin of the metapleuron $(\mathrm{C})$. The third axillary is a large, elongate sclerite $\left(\mathrm{B}, 3^{A \mathrm{x}}\right)$ lying obliquely transverse behind the second axillary (A) between the edge of the metanotum and the base of the fourth wing vein. The large, fan-shaped, three-branched muscle of the wing-flexing third axillary $(\mathrm{B}, \mathrm{F}, \mathrm{G}, \mathrm{H}, I 00)$ arises on the anterior infold of the metapleuron $(\mathrm{C}, \mathrm{H}, k)$ and is attached, not directly on the third axillary, but on a minute sclerite of the wing membrane ( $\mathrm{A}, \mathrm{B}, a$ ) close to the base of the axillary, and hence pulls indirectly on the latter through the intervening membrane.

The epipleurites.-The epipleural sclerites associated with the under sides of the wing bases include in each segment the basalare lying before the pivotal second axillary, and the subalare lying behind it. The sclerites are articulated on the upper edges of the pleura; they give insertion to muscles that deflect them inwardly and thus pull on the wing bases by reason of the close membranous connection of the epipleurites with the latter. The specific effect of the epipleurite muscles on the wing movements will be described later.

The basalare of the mesothorax (fig. I9 $\mathrm{B}, B a$ ) is an elongate sclerite hinged by its entire length on a wide lobe $(d)$ of the dorsal margin of the mesopleuron, which supports posteriorly also the second axillary $(2 A x)$. On the inner surface of the basalare is attached by a strong apodemal tendon a large, flat, fan-shaped muscle $(\mathrm{G}, 77)$ arising on the anterior part of the pleuron (fig. I $7 \mathrm{E}$ ), where it partly overlaps internally the second and third muscles of the third 
axillary $(76 b, 76 c)$. The contraction of this muscle turns the basalare inward on the supporting pleural lobe, and the movement is transmitted to the wing because of the close connection of the basalare with the humeral angle of the wing base.

The subalare of the mesothorax is a small triangular sclerite (fig. I9 I, $\mathrm{Sa}$ ) but little visible in lateral view (B) beneath the posterior part of the wing. Its broad base is supported on a longer sclerite (I, $h)$, which in turn rests on the inner margin of the inflected dorsal plate of the epimeron $\left(E p m^{\prime}\right)$, and is linked posteriorly with the anterior extremity of the stalk of the postphragma $(2 P h)$. On the upper angle of the subalare is attached the tendon of a long muscle (82), which arises ventrally on the mesothoracic coxa (figs. I7 G, $22 \mathrm{C}, 82)$. The subalare is closely associated with the posterior basal process of the second axillary sclerite (fig. I9 B), so that the pull of its muscle is indirectly transmitted to the pivotal sclerite of the wing base.

The epipleural sclerites of the metathorax have the same relations to the hind wing as do those of the mesothorax to the fore wing. The triangular basalare (fig. 2I $\mathrm{B}, B a$ ) sits on a marginal elevation of the metapleuron before the second axillary $(\mathrm{C}, \mathrm{Ba})$, and the small subalare $(\mathrm{B}, S a)$ is attached to the concave pleural margin behind the second axillary $(\mathrm{C}, \mathrm{Sa})$. A single large basalar muscle $(\mathrm{H}, \mathrm{IOI})$ arises on a lobe $(r)$ of the ridge in the metapleural area behind the middle leg (fig. I6 $\mathrm{H}, r$ ), and tapers upward to a strong tendon attached mesally on the basalar sclerite (fig. 2I B, $\mathrm{H}, \mathrm{Ba}$ ). The small, inconspicuous subalare $(\mathrm{B}, S a)$ has an elongate apodemal process on which are inserted two muscles, one a long fusiform muscle (IO5), corresponding with the subalar muscle of the mesothorax, which arises ventrally on the hind coxa (fig. $24 \mathrm{~A}, 105$ ), the other a broad thick muscle (fig. 2I B, C, H, IO2) arising on the upper plate of the metapleuron, and apparently represented in the mesothorax by a similar pleural muscle (fig. I7 E, 75) attached dorsally on the margin of the posterior scutal plate of the mesonotum (fig. I9 F, 75). The subalare is intimately associated with the second axillary of the wing base (fig. 2I B, 2Ax) by a close membranous connection (b) with the posterior basal arm of the latter.

The mechanism of flight.-To understand the movements of an insect's wings it is necessary to observe first that the wings are supported from below on the second axillary sclerites of their bases, and that these sclerites rest on the upper edges of the pleura, while the flexible attachments of the wings to the notal plates lie a little mesad of the pleural supports. As a consequence a slight depression of the 

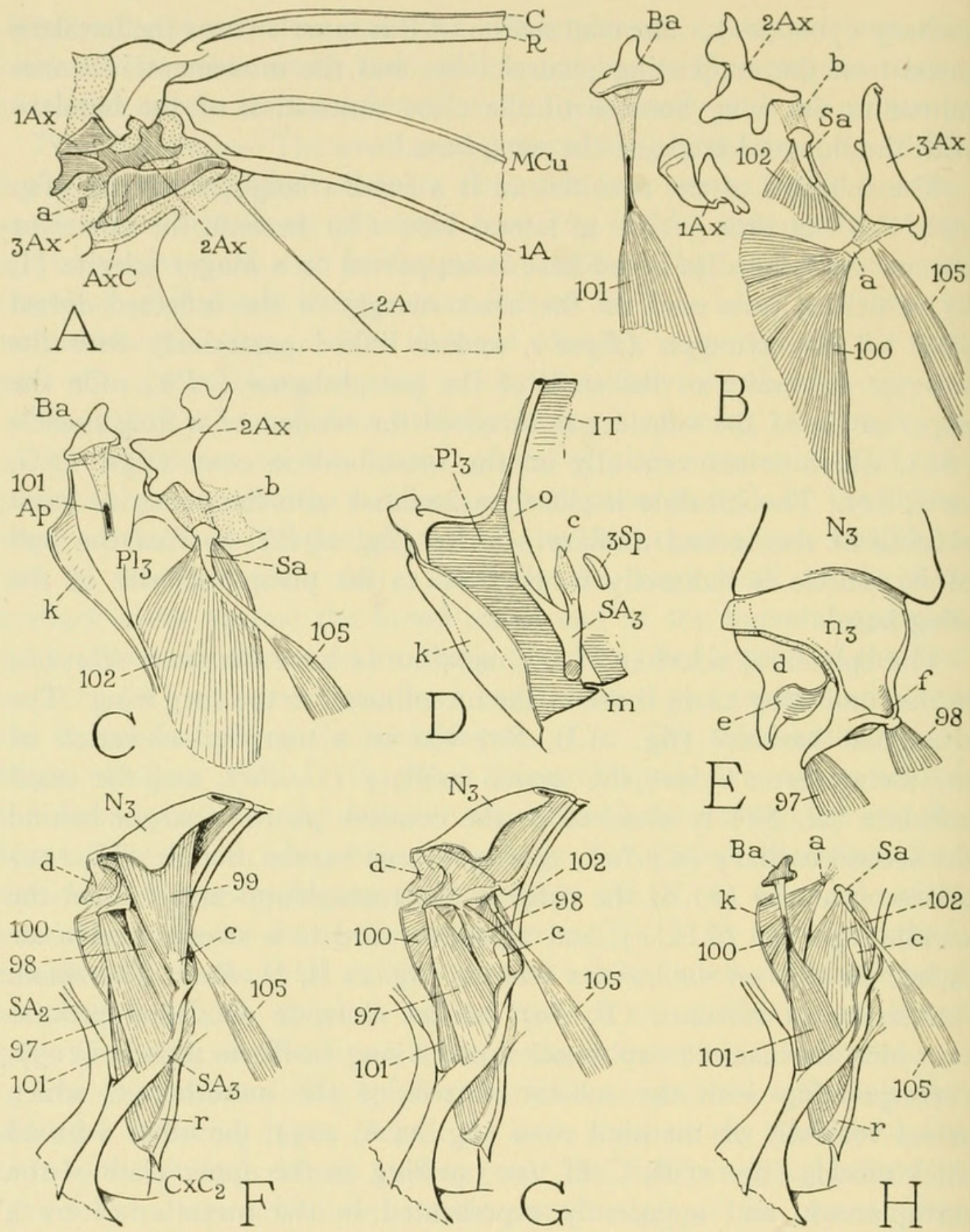

FIG. 2I.-Details of the metathorax, base of the hind wing, and the lateral metathoracic muscles (drone).

A, base of hind wing, dorsal view. B, axillary, basalar, and subalar sclerites of hind wing, dorsal view. C, inner face of upper part of right metapleuron, showing pleural articulations of basalare, second axillary, and subalare, and muscles of subalare. D, upper part of right metapleuron and adjoining part of propodeum, anteromesal view. E, lateral part of metanotum, left side. $\mathrm{F}$, lateral muscles of metathorax, right side, mesal view. G, same, with third tergosternal muscle (99) removed. H, same, with notum and first, second, and third tergosternal muscles $(97,98,99)$ removed, exposing basalar and subalar muscles $(I O I, I O 2, I O 5)$ and muscle of third axillary $(I O O)$.

$a$, nodule of wing membrane giving insertion to muscle of third axillary; $b$, "ligament" of wing membrane connecting second axillary with subalare; $c$, process of metathoracic endosternal arm $\left(S A_{3}\right)$ giving attachment to third tergosternal muscle (99); $d$, free marginal sclerite of metanotum; $e$, lobe of $d$ supporting head of first axillary; $f$, posterior articular socket of first axillary; $k$, anterior inflection of metapleuron; $m$, groove between metapleuron and propodeum; $n_{3}$, semidetached lateral plate of metanotum; $o$, internal ridge between metapleuron and propodeum, $r$, lobe giving attachment to basalar muscle (IOI). 
lateral margins of the back plates of the wing-bearing segments causes the wings, in the manner of a pump handle, but involving the interaction of many structural details, to turn upward, and an elevation of the notal margins tilts the wings downward. The up-anddown movements produced in this way, however, are not sufficient for progressive flight; in addition the wings must have a partial rotary movement on their long axes accompanied by a forward and rearward motion, by which the anterior margins are turned downward and forward during the downstroke, and reversed during the upstroke. This compound motion gives a sculling movement to each wing, but the result of the wings on opposite sides of the body acting together is that a current of air is driven backward, and the insect propelled forward. In the bee the two wings of each side act in unison as a single pair of flight organs because of their connection with each other, but the unity of action is further secured by a reduction of the metathoracic musculature and its subordination to the action of the highly developed mesothoracic muscles, which latter thus become the chief motor elements for both pairs of wings.

The cavity of the pterothorax is largely occupied by the huge dorsoventral and dorsal longitudinal muscles of the mesothorax (fig. I6 A, C). The thick pillars of dorsoventral muscles $\left(7^{2}\right)$ have their upper attachments (A) on the lateral parts of the anterior plate of the mesonotum (B), and go downward and backward (C) to their lower attachments on the ventral and lower lateral walls of the pleurosternal region of the mesothorax. The longitudinal muscles $\left(7^{I}\right)$ are attached anteriorly on the prephragma and the median area of the anterior notal plate (B), and extend posteriorly through the metathorax into the posterior end of the propodeum, where they are attached on the postphragma $(2 P h)$. Though the postphragma of the bee is extended through the metathorax far back into the propodeum, to accommodate the great length of the mesothoracic dorsal muscles, it is morphologically an ingrowth between the mesonotum and the metanotum. A pair of small fan-shaped muscles $(\mathrm{C}, 96)$, arising on a median process of the posterior end of the propodeal tergum (IT) and spreading to their insertions on the rear surface of the phragma, must represent, therefore, the usual longitudinal dorsal muscles of the metathorax with their posterior attachments transposed backward on the propodeum. A pair of short external dorsal muscles of the mesothorax $\left(\mathrm{D}, 7^{\circ}\right)$ traverses the scutellar area of the mesonotum.

The vertical component of the upstroke of the fore wings, resulting from a depression of the lateral margins of the mesonotum, is effected principally by the great dorsoventral muscles (fig. I6 C, 72), but 
probably important adjuncts are the large pleuronotal muscles arising on the sides of the pleuron (fig. I7 E, 75) and attached on the ends of the posterior scutal areas of the notum (fig. I9 F, 75). The wing bases, hinged to the back by the first and fourth axillary sclerites, are pressed downward proximal to the fulcral second axillaries by the descending margins of the notum, and thereby the distal parts of the wings are thrown upward. The vertical component of the downstroke, conversely, results from the contraction of the longitudinal muscles, which restores and increases the curvature of the mesonotum, and thus turns the wings down on the fulcral sclerites.

In most insects the wing-bearing plates of the back are sufficiently thin and flexible to respond by changes in shape to the alternating pull of the vertical and longitudinal muscles. Such movements, however, are not possible in the thick and rigid mesonotum of the bee and related Hymenoptera, and it is for this reason that the mesonotum is cut by a transverse fissure (fig. I6 C, $s f$ ) into an anterior and a posterior plate. The fissure widens on each side into an open cleft (fig. I9 J, sf), the edges of which are connected by an infolded membrane. The closed dorsal part of the fissure acts as a hinge between the two plates. Pressure on the top of the back depresses the notal plates at the hinge line and widely opens the lateral clefts, the anterior notal plate here moving forward, the posterior plate backward. The divergent movement of the two plates, however, accentuates the downward movement of their lower margins at the points just before and behind the lateral clefts where the wings are articulated to the back by the first and fourth axillary sclerites. This action of the mesonotum is easily demonstrated by manipulation of a dead bee, and could be well illustrated with half of a hollow rubber ball having a meridional slit on each side.

In the chalastogastrous Hymenoptera the posterolateral areas of the mesoscutum form deep concavities of the scutal surface at the sides of the elevated median area of the scutellum, and are separated from the main part of the scutal plate before them by the lines of abrupt deflections of the notal surface. These lines coincide with the scutal clefts of the Clistogastra, and, in fact, each is split laterally by a short cleft. The response of the relatively flat mesonotum of a tenthredinid to gentle compression between its two ends is most pronounced on the sides; the anterior wing processes spread laterally and move upward and posteriorly, the lateroposterior margins of the anterior scutal areas at the lateral clefts folding backward above the anterior edges of the posterolateral areas. In the densely sclerotic and strongly convex mesonotum of the Clistogastra a much deeper splitting 
of the scutum has become necessary for carrying out the mechanical function of the notum. The scutal clefts of the Hymenoptera in any case, are simply extensions of the usual lateral emarginations of a wing-bearing notum that are bridged by the first axillary sclerites of the wing bases.

The opening of the scutal clefts by pressure on the back causes a posterior displacement of the postphragma in the propodeum because of the connection of the phragma with the scutellum (fig. I6 C), and this movement of the phragma must stretch the longitudinal muscles preparatory to their succeeding contraction. The contraction of the longitudinal muscles $(7 I)$, extending from the anterior notal plate to the phragma, not only closes the lateral clefts of the notum, but causes the edges of the posterior plate to slide beneath those of the anterior plate, thus increasing the curvature of the back to give greater effectiveness to the downstroke of the wings. The small propodeal muscles (96) attached on the back of the phragma may be supposed to contribute to the movement of the phragma, though their narrowed and convergent posterior ends (fig. $27 \mathrm{C}, 96$ ) would suggest that they pull on the propodeum. The function of the pair of delicate muscles in the mesothoracic scutellum (fig. I6 D, $7^{\circ}$ ) is not clear.

The metathorax contains no muscles corresponding in function with the dorsal muscles of the mesothorax, since the only intersegmental dorsal muscles that can be referred to the metathorax are the greatly displaced pair between the postphragma and the propodeum (fig. I6 C, 96). The dorsal muscles of the mesothorax $(7 I)$, therefore, must cause the downstroke of both pairs of wings when the wings of each side are connected. Probably in the same way the dorsoventral muscles of the mesothorax are largely responsible for the upstroke of the metathoracic wings, since as long as the wings are connected pressure on the mesonotum alone lifts both pairs of wings, though it has no effect on the hind wings when the latter are unhooked. However, the metanotum itself is amply provided with depressor muscles, which very probably in the living bee play an important part in the elevation of the wings.

In each side of the metathorax there are three large muscles attached on the notum, which arise ventrally on the metathoracic arm of the pterothoracic endosternum. The first two of these muscles (fig. 2I F, G, 97, 98) arise by broad bases on the endosternal arm and taper upward to their dorsal attachments on the lower edge of the semidetached lateral plate of the notum $\left(\mathrm{E}, n_{3}\right)$. The third and largest muscle $(\mathrm{F}, 99)$ arises from a small lobe $(c)$ of the endosternal arm, and its fibers spread upward to their attachments on the lateral 
triangular area of the dorsal part of the notum. A downward pressure on this region of the metanotum at once turns the hind wings upward, leaving little doubt that the metanotal muscles are wing elevators.

The horizontal and rotary components of the wing motion, by which the wings are turned forward with the costal margins deflected during the downstroke, and reversed during the upstroke, are produced chiefly by the muscles of the basalar and subalar sclerites. It is these movements that convert the wings from mere flaps into organs of propulsion. The mechanism involved is equally developed in each wing-bearing segment of the bee. The epipleural sclerites, as already explained (figs. I9 B, I, 2I C, Ba, Sa), are hinged to the upper edges of the pleura in such a manner that the contraction of their muscles turns them mesally in the subalar membranes. The movements of the sclerites are effective on specific points in the wing bases because a tract of the membrane intervening between each sclerite and the wing is so tightly stretched that it acts as a direct union, and often gives a superficial appearance of being a connecting ligament. The basalare in each wing of the bee is thus connected with the humeral complex of the wing base, and the subalare with the posterior lever arm of the second axillary (fig. 2I B). Contraction of the basalar muscle during the downstroke of the wing, therefore, revolves the basalare inward on its pleural hinge, and the tension exerted on the wing base turns the descending wing forward on the pivotal second axillary and deflects its costal margin. Similarly, during the upstroke, the subalar muscle acting on the subalare pulls on the lever arm of the second axillary and thus tilts the wing downward posteriorly, so that the costal margin turns upward and moves backward, relative to the body, while the wing is ascending.

Flexion and extension of the wings.-When the bee alights after flight, or when the organs of flight are not otherwise in use, as in "fanning," the wings are flexed posteriorly, the hooks on the hind wings automatically loosing their grasp on the fore wings, and both pairs of wings are laid over the back, the fore wings on top of the hind wings. The mechanical problem involved in the horizontal flexing of an insect's wing may be illustrated with a piece of paper firmly held flat at one end and bent horizontally on itself; the bending necessarily produces a fold across the base. Conversely, if the paper is folded in the same way, it automatically bends backward at a right angle to its flat position. The flexing apparatus of the wing acts on the second principle-by the production of a basal fold the wing is turned horizontally backward. The fold-producing mechanism is the 
third axillary sclerite and its muscles, but in the fore wing of the bee the action of the third axillary appears to be assisted by the fourth axillary and its lever.

The basal region of the fore wing of the bee in the natural position of extension (fig. I9 A) is rolled on itself in such a way that most of the axillary area is deflected. There is hence an oblique fold in the wing base extending from the head of the first axillary $(I A x)$, behind the second axillary and the median plate, to the upwardly directed distal end of the third axillary $(3 A x)$. The pull of the third axillary muscles $(\mathrm{H})$, therefore, simply revolves the sclerite mesally and somewhat forward on its base and thus accentuates the fold already present, with the result that the distal part of the wing turns backward. If the wing base were flat (D), as it is in most insects, the action would not be essentially different, as can be demonstrated by revolving the third axillary in the wing of a dead specimen.

The part that the fourth axillary plays in the flexing of the fore wing of the bees can be shown by depressing its lever (fig. I9 D, $a x l v)$. The fourth axillary $(4 A x)$ is movably articulated on the margin of the mesonotum and its distal end is pressed against the axillary cord $(A x C)$. The head of the lever lies in the wing membrane immediately behind the fourth axillary. A downward pull on the lever muscle (fig. $20 \mathrm{~A}, 7^{8}$ ) depresses the lever on its fulcral connection $(o)$ with the end of the postphragma, and causes the head $(s)$ to pull backward on the fourth axillary $(4 A x)$. The axillary turns on its notal articulation and its outer end pulls on the axillary cord. The wing gives a quick response with a slight backward movement. Slight though this movement of the wing is, the elaborateness of the mechanism that produces it suggests that the movement is one of considerable importance. It may be seen, in fact, that the backward jerk on the extended wing brings the third axillary into a more nearly vertical position in which it becomes quickly responsive to the action of its own muscles. The fourth axillary and its lever in the fore wing of the bee, therefore, appear to be a starting apparatus for the flexor mechanism.

The flexing of the hind wing is produced entirely by the large third axillary sclerite and its muscle (fig. 2I A, B, $3 A x$ ), since there is no fourth axillary or lever accessory. The axillary region of the hind wing, however, unlike that of the fore wing, is approximately flat when the wing is extended (A). It is probable, therefore, that on relaxation of the extensor muscle the wing automatically turns backward sufficiently to raise the third axillary into a position in which its muscle can become effective. 
The extension of the flexed wings preparatory to flight is in general produced by the basalar sclerites and their muscles. In a dead specimen a slight pressure on the basalare of either wing, or a pull on its muscle, brings the wing out at right angles to the body and deflects its anterior margin. The fore wing of the bee, however, is provided with a small pleuroalar muscle (fig. I9 C, 74), which arises on the anterior marginal carina of the mesopleuron (fig. I7 C, 74) just below the attachment of the spiracular muscle (73), and extends dorsally and posteriorly to its insertion by two branches attached ventrally on the humeral complex of the wing base (fig. I9 C). This muscle evidently must be an accessory wing-extensor. In some insects a depression of the second axillary, produced by the pull of the subalar muscle, helps to extend the wing, but an extensor action of the second axillary cannot be demonstrated in the bee.

\section{THE LEGS}

The legs of Hymenoptera have the usual six segments of an insect leg, namely a coxa, a trochanter, a femur, a tibia, a tarsus, and a pretarsus. A leg segment is to be defined as a section of the limb independently movable by muscles attached on its base, except where there is evidence of the union of segments or a suppression of muscles. The tarsus is usually divided into subsegments, or tarsomeres, which, though movable on each other, are not "tarsal segments" since only the proximal piece is provided with muscles. The flexible areas between segments or subsegments are the joints of the leg. The proximal tarsomere, especially when differentiated by size or other features, as in the bee, is designated the basitarsus. The pretarsus either terminates in a single claw, or dactyl, or it bears a pair of lateral claws, or ungues, and usually a median lobe, the arolium.

The three pairs of legs of the bee are attached at somewhat different angles on the thorax so that in their action they are radially distributed at the sides of the body. The coxae of the fore legs hang downward on transverse axes of movement (fig. II G, $C x$ ), and hence the first legs swing forward and backward in the transverse planes of the coxae. The middle coxae are transversely elongate and set obliquely into the under wall of the thorax (figs. I $5, \mathrm{I} 6 \mathrm{~F}$ ), and the trochanters are attached at their mesal ends; the middle legs, therefore, are limited in their up-and-down motion, and swing forward and backward by the revolution of the coxae on the body. The hind coxae also have obliquely transverse axes on the thorax (figs. I5, I6 F), but they are directed posteriorly and hence give the hind legs a more 
backward position at the sides of the abdomen. Each leg has characteristic features, and, in the worker particularly, various parts of the legs are developed into specific structures for special uses.

For descriptive purposes it is customary to name the leg surfaces according to the positions they assume when the limb is extended straight out from the body. In such a position the upper surface is dorsal, the under surface ventral, the preaxial surface anterior, and the postaxial surface posterior. The actual directions of the leg surfaces in normal positions, however, may be quite different.

Inasmuch as the legs differ principally in relative size, form, and outer structure, the general external features will be described separately for each pair, while the mechanism of the joints, and the muscles, which are but little different in the three pairs of appendages, will be treated collectively in order to avoid unnecessary repetition.

The fore legs.-The fore legs (fig. I3 A) are smaller than the others and have somewhat less freedom of motion because of the vertical position of the coxae (fig. I I G), but, in compensation, the femur of each fore leg has an anterior twist (fig. I4 A) by which the tibia acquires a forward movement, and, when fully flexed, lies against the anterior surface of the femur and trochanter. The long, cylindrical, bristly first tarsomere is used as a brush for removing pollen or other particles from the head and fore parts of the body, but the most important special features of the first leg is the antenna cleaner at the base of the tarsus.

The antenna cleaner (fig. I4 B) consists of a deep semicircular notch $(b)$ on the inner (ventral) face of the proximal end of the basitarsus (Btar), and of a large flattened spur $(a)$ that serves as a clasp, or fibula, projecting from the inner angle of the distal end of the tibia $(T b)$. The posterior margin of the notch bears a comblike fringe, or pecten, of fine, closely set, spinelike hairs. The fibula is a broad, thin, movable appendage with a narrowed base, a tapering distal point, and a strong, spatulate accessory lobe $(e)$ on its anterior surface. When the tarsus is flexed by the depressor muscle (E, 68) the proximal lobe of the basitarsal notch $(\mathrm{C}, c)$ glides under the accessory lobe $(e)$ of the fibula, and the distal point of the tarsal notch $(d)$ is caught in the angle between the two fibular lobes. The tarsal notch is thus closed to a semicircular aperture by the accessory lobe $(e)$ of the fibula, and the larger flat lobe $(a)$ overlaps the aperture posteriorly. When the organ is used by the bee, the tarsal notch is placed around the base of the antennal flagellum by movements of the leg, and then, by flexion of the tarsus, the antenna is pressed against the accessory lobe of the fibula, which latter resists the 
pressure because of a small process of the tibia $(f)$ that abuts against its base. The antenna is thus held securely and is now drawn upward through the cleaner, the tarsal comb brushing its sensory outer surface, the thin lobe of the fibula scraping its inner surface.

The antenna cleaner is not a gadget peculiar to the bees; it is developed in some measure in most of the clistogastrous Hymenoptera (fig. I4, F, G, H, I), though the tarsal notch is usually shallower than in the honey bee, and may be but a sinuosity of the tarsal margin. The fibula of the cleaner is simply a special development of an ordinary movable spur usually present on the end of the tibia, such as is present on the middle leg of the adult bee (fig. $22 \mathrm{~A}, \mathrm{~B}$ ), and also on the hind leg of the pupa.

The middle legs.-The second legs are the least specialized of the legs in the bee, and have the simplest form in the drone (fig. $22 \mathrm{~A}$ ). In each caste the end of the tibia is armed ventrally with a long spine, which has been said to be used by the worker for removing wax from the wax pockets of the abdomen, but the evidence of such use of the spine has been questioned. The basal tarsomere is long and cylindrical in the drone (A), in the worker and queen it is wide and flattened, in the worker (B) its under surface is thickly covered with long stiff hairs projecting distally, and serves as a pollen brush.

The hind legs.-The large hind legs are the most specialized in form of all the legs by reason of the great size and lateral flattening of the tibia and basitarsus (fig. $23 \mathrm{~A}, \mathrm{~F}, \mathrm{H}$ ). Though this feature is common to the three castes, a functional reason for it is not evident except as it is elaborated for useful purposes in the worker. The femur has a coating of small hairs in the drone $(\mathrm{F})$, in the queen and the worker (A) it is bushy with long feathery hairs. The tibia of the drone $(F)$, aside from its size and shape, has no special characters; in the queen $(\mathrm{H})$ the tibia is relatively wider and thick at its base; in the worker (A) it is narrowed proximally and expanded distally, and its smooth, somewhat concave outer (anterior) surface (C) is bordered with long curved hairs, forming the pollen-carrying basket, or corbicula. The basitarsus is equally large in each caste $(\mathrm{A}, \mathrm{F}, \mathrm{H})$, but there is no apparent reason for its size and shape in the queen and the drone; the cavity of the basitarsus is traversed by the tendon of the pretarsal claws and is otherwise occupied only by a loose mass of fat tissue. In the worker the inner (posterior) surface of the basitarsus (A, Btar) is armed with about Io transverse rows of long, slender spines directed mesally and distally at an angle of $45^{\circ}$. The basitarsus of the worker thus resembles a flat brush or currycomb, and has been termed the strigilis (Betts, 1923), though the same 

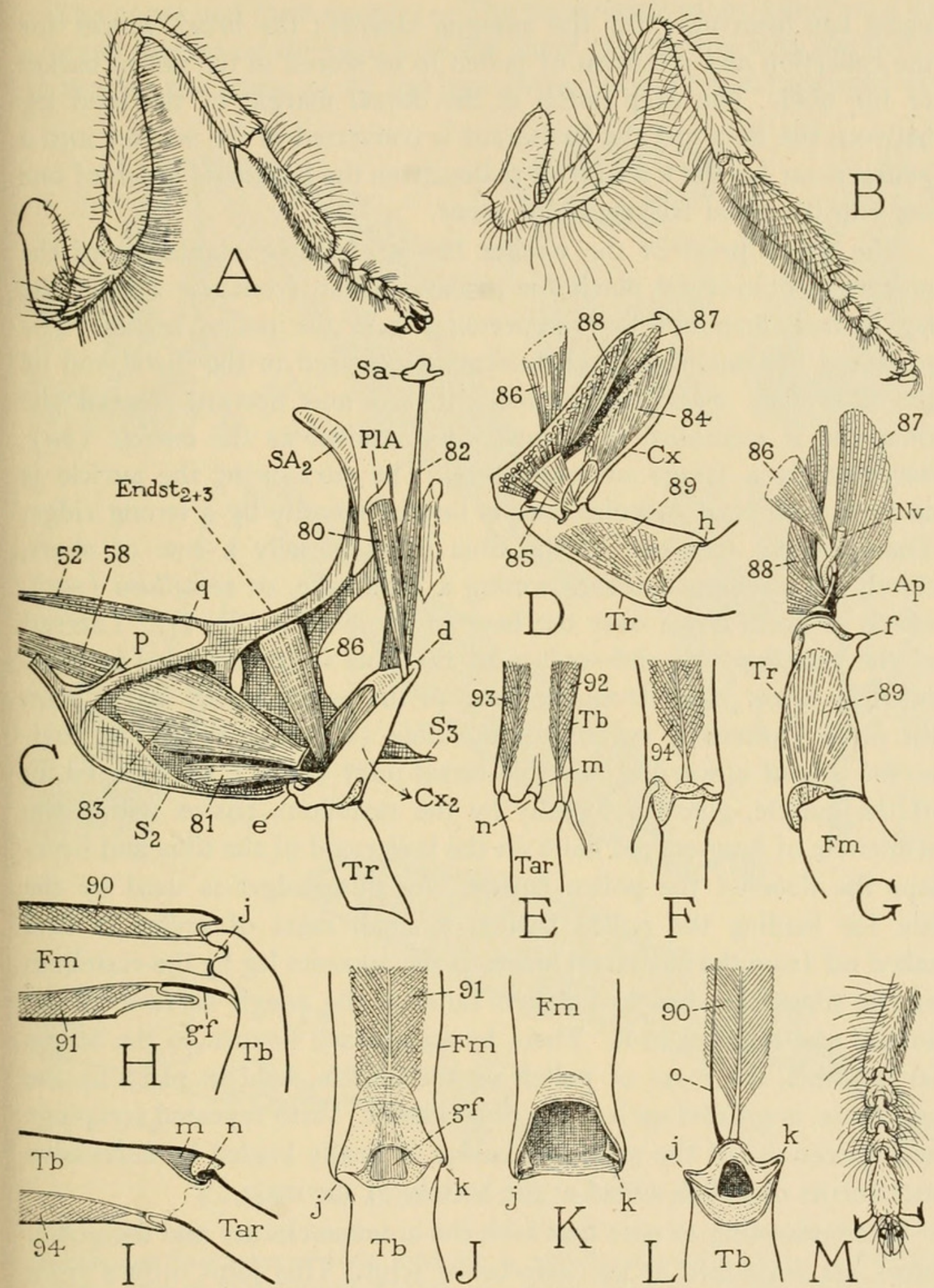

FIG. 22.-The mesothoracic leg and its muscles.

A, right middle leg of drone, posterior view. B, same of worker, posterior view. C, endosternum of pterothorax and mesothoracic coxa, with thoracic muscles of leg, left side (coxa turned outward). D, base of right middle leg of drone, posterior view, showing trochanteral and femoral muscles. E, tibio-tarsal joint of right middle leg of drone, dorsal view. F, same, ventral view. G, trochanter and base of femur of left middle leg of drone, with muscles, mesal view. H, diagram of dicondylic femoro-tibial joint. I, diagram of monocondylic tibiotarsal joint (lateral muscles not shown). J, femoro-tibial joint of right middle leg of drone, ventral view. $K$, end of femur with tibia removed, ventral view. L, base of tibia, ventral view, showing levator muscle $(90)$ and chordotonal ligament $(o)$. M, tarsus and pretarsus of right middle leg of drone, dorsal view.

$d$, pleural articulation of coxa; $e$, sternal articulation of coxa; $f$, anterior coxal articular process of trochanter; $h$, dorsal articulation of trochantero-femoral joint; $j$, anterior femorotibial articulation; $k$, posterior femoro-tibial articulation; $m$, tibial articular process of tibiotarsal joint; $n$, tarsal articular knob of tibio-tarsal joint; $o$, tendon of femoral chordotonal organ; $p$, process of endosternum giving attachment to muscle $58 ; q$, process of supraneural bridge of endosternum giving attachment to muscle 52 . 
name has been used for the antenna cleaner; the brush serves for the collection and retention of pollen to be stored in the pollen basket of the tibia. The deep notch in the dorsal margin of the hind leg between the tibia and the basitarsus is converted in the worker into a pollen press for the transfer of pollen from the basitarsal brush of one leg into the tibial basket of the other.

The pollen press of the worker bee is a simple adaptation of the pincerlike tibio-tarsal notch for pushing pollen from one side of the leg to the other and for compressing it in the pollen basket. The proximal end-surface of the basitarsus opposed to the distal end of the tibia (fig. 23 B) is beveled outward and upward toward the tibia and is expanded in a small lobe, known as the auricle (au), bordered by a fringe of long hairs. The surface of the auricle is covered with small spicules, and is limited mesally by a strong ridge. The opposing free end of the tibia bears mesally a row of short, closely set, sinuous spines, forming a little rake, or rastellum (ras), which projects down over the base of the auricle (D, E). Laterad of the rastellum the end-surface of the tibia is concave and is separated by a low ridge from the floor of the pollen basket above it on the outer (anterior) surface of the tibia $(\mathrm{C}, c b l)$. When the basitarsus is bent upward at the tibio-tarsal joint $(\mathrm{D}, \mathrm{E})$, the fringed lip of the auricle, guarded mesally by the rastellum, passes within the semicircle of long curved hairs on the lower end of the tibia and overlaps the floor of the pollen basket. As this gadget is used by the bee for loading the pollen basket, a small mass of pollen is first raked off from the basitarsal brush of the opposite leg by the rastellum of the tibia; the detached pollen falls on the rough surface of the auricle and adheres to it. Then, by an upward flexure of the tarsus on the tibia, the mass of pollen on the auricle, held in place by the rastelium, is pressed up into the tibial basket. With repeated scrapings and operations of the press the basket is finally loaded by successive increments of pollen added at the bottom of the mass.

It is interesting to note that both the antenna cleaner and the pollen press are developed at the tibio-tarsal joint. This joint differs from the other leg joints in that the tarsus has a monocondylic hinge on the tibia, and three muscles. Since this structure pertains to the corresponding joint in all the legs of the three castes of the bee, it is clear that it is not an adaptation to either the antenna cleaner or the pollen press. On the other hand, it is possible that the development of these instruments has depended on the peculiar nature of the tibiotarsal joint and the unusual mobility of the tarsus. 
The coxae and their muscles.-The movements of an insect's leg as a whole depend on the nature of the coxal articulation with the body. In the bee the coxae of all the legs swing forward and backward on

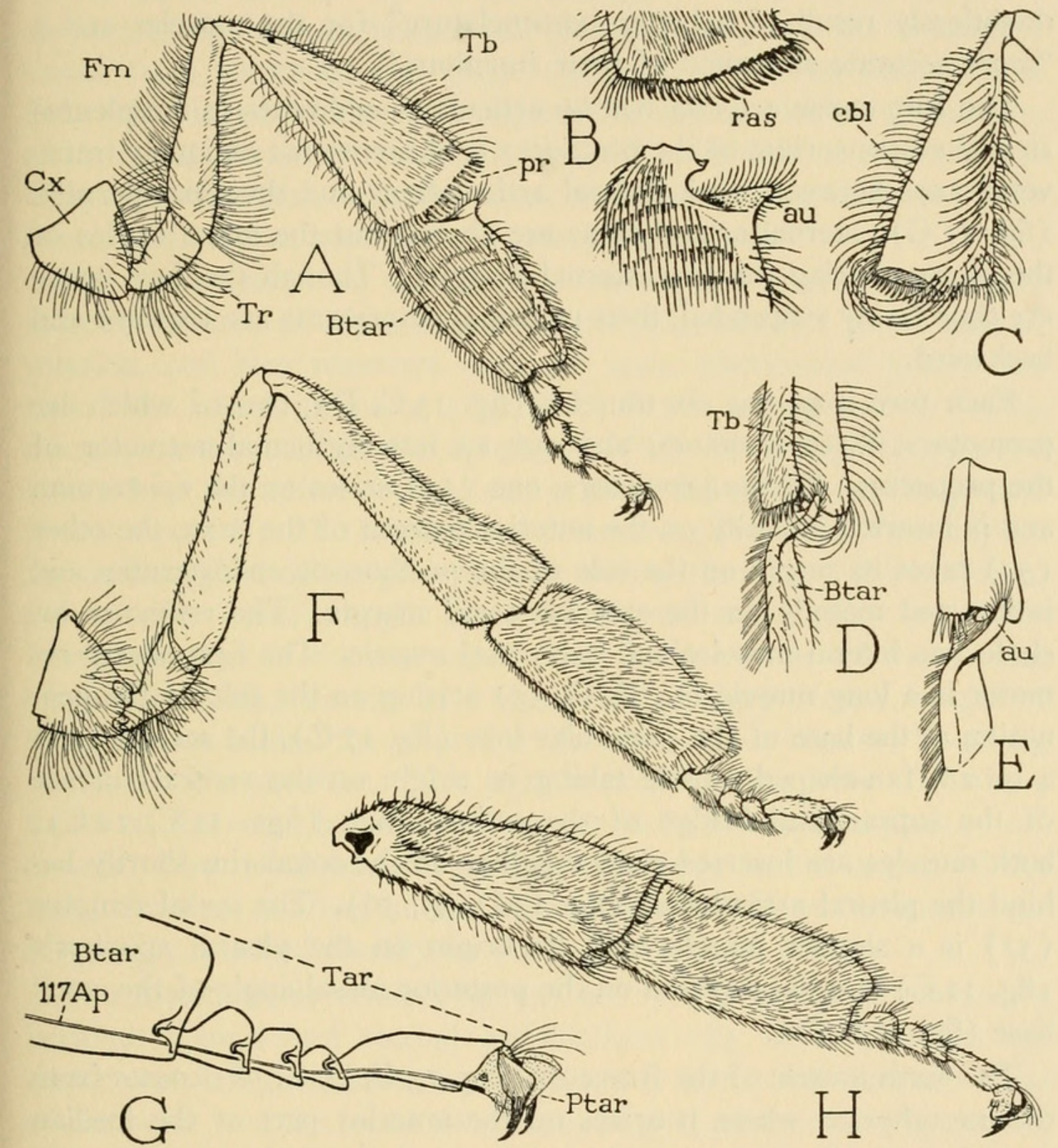

FIG. 23.-The metathoracic leg, external structure.

A, right hind leg of worker, posterior (inner) surface. B, opposing ends of tibia and basitarsus of right hind leg of worker, posterior view. C, anterior (outer) face of hind tibia of worker. D, pollen press of worker between tibia and basitarsus, dorsal view. E, same, better shown by removal of tibial hairs. $\mathrm{F}$, right hind leg of drone, posterior (inner) surface. $\mathrm{G}$, right hind tarsus and pretarsus of drone, posterior view. $\mathrm{H}$, right hind tibia, tarsus, and pretarsus of queen, posterior surface.

transverse axes, and the muscles of each coxa are inserted anterior and posterior to the coxal axis of movement. The coxal muscles, therefore, are promotors and remotors. Morison (1927) designates 
the coxal muscles "extensors" and "flexors," the "extensors" being the lateral muscles, the "flexors" the mesal muscles, but this nomenclature ignores the relation of the muscles to the natural movements of the coxae. Morison adds, however, that a further study "would doubtlessly result in a better nomenclature" for the muscles and a "more accurate statement of their functions."

The fore coxae are attached by articular membranes to the pleurosternal suspensorium of the prothorax with somewhat obliquely transverse axes between weak pleural articulations and the furcasternum (fig. II G) ; sternal articulations are absent, but the mesal angles of the coxae overlap the furcasternal margins. Though the fore coxae are thus freely suspended, their principal movements are forward and backward.

Each fore coxa has six muscles (fig. I3 C, D), two of which are promotors, three remotors, and one an intersegmental retractor of the propectus. Of the promotors, one (53) arises on the episternum and is inserted laterally on the anterior margin of the coxa, the other (54) takes its origin on the side of the prothoracic endosternum and is inserted mesally on the anterior coxal margin. The remotors include two lateral muscles and one mesal muscle. The first lateral remotor is a long muscle $(\mathrm{C}, \mathrm{D}, \mathrm{G}, 55)$ arising on the side of the pronotum at the base of the spiracular lobe (fig. I7 C), the second ( $5^{6}$ ) a broad, fan-shaped muscle taking its origin on the ventral surface of the supraneural bridge of the endosternum (figs. II C, I2 C); both muscles are inserted close together on the coxal rim shortly behind the pleural articulation (fig. I $3 \mathrm{D}, 55,56$ ). The mesal remotor (57) is a slender muscle with its origin on the pleural apophysis (fig. I I C) and its insertion on the posterior mesal angle of the coxal base (fig. I3 C, D).

The sixth muscle of the fore coxa (fig. $\mathrm{I} 3 \mathrm{C}, \mathrm{D}, \mathrm{G}, 5^{8}$ ) comes from the mesothorax, where it arises on the anterior part of the median sternal ridge that supports the superstructure of the pterothoracic endosternum (fig. 22 C) ; it extends horizontally forward and laterally (fig. I2 $\mathrm{C}, 58$ ) to its insertion on a small point of the coxal base mesad of the pleural articulation (fig. $\mathrm{I}_{3} \mathrm{D}$ ). The pull of this muscle can have little specific motor action on the coxa, but the two muscles diverging from the mesosternal ridge to the fore coxae are evidently retractors not only of the coxae but of the entire propectus, being thus adjuncts of the intersegmental ventral thoracic muscles (fig. I2 C, $5^{2}$ ). The intersegmental coxal muscle is classed as an "extensor" of the coxa by Morison (I927), but the corresponding muscle in Vespula 
is termed by Duncan (1939) the "mesosternal retractor of the propectus."

The coxae of the mesothorax are elongate and semicylindrical in form; each is attached by the entire length of one side to the under surface of the thorax behind the mesosternum, and the trochanter arises from its mesal end (figs. I5, 22 A, B). The mesocoxa is definitely hinged to the body between a lateral pleural articulation and a mesal sternal articulation (fig. I6 F) ; its axis of movement is obliquely transverse, with the mesal end somewhat in advance of the lateral end, and its movement is rotary on the axis.

The musculature of each middle coxa comprises two promotor muscles inserted at opposite ends of the coxa anterior to the axis of rotation, and two remotors with the same distribution behind the axis. The lateral promotor (fig. $22 \mathrm{C}, 80$ ) is a long muscle arising dorsally on the small pleural apophysis $(P l A)$ of the mesothorax, and inserted on the anterior coxal rim a short distance from the pleural articulation $(d)$. The small mesal promotor $(\delta I)$ arises ventrally on the median plate of the pterothoracic endosternum, and is inserted on the anterior coxal margin near the sternal articulation $(e)$. The lateral remotor of the mesocoxa $(82)$ is the muscle of the subalar epipleurite $(S a)$; it arises by a broad base on the posterior margin of the coxa just behind the pleural articulation, and is inserted by a long tendon on the subalare. This muscle probably has little effect as a motor of the coxa, but it would appear to be primarily a pleurocoxal muscle. The mesal remotor of the coxa $\left(8_{3}\right)$ is the largest of the coxal muscles; it arises on the median plate of the endosternum and is strongly attached mesally on the posterior coxal margin.

The metathoracic coxae (fig. I5) are hinged obliquely transverse between pleural and sternal articulations (fig. I6 F), as are the mesocoxae, but their bases are more nearly horizontal than are those of the latter, and the coxae themselves project posteriorly (fig. $24 \mathrm{~A}$ ), with the trochanters arising from their distal ends.

The musculature of the metacoxae is the same as that of the mesocoxae, there being inserted on the base of each hind coxa four muscles (fig. 24 B), two of which, attached before the coxal axis $(d-e)$, are, the one a lateral promotor (IO3), the other a mesal promotor ( 104 ), while a third inserted mesally behind the axis is a mesal remotor (I06). The fourth muscle (I05), as in the mesothorax, is the coxosubalar muscle of the wing; it is attached on the coxa so close to the pleural articulation $(d)$ and so nearby on the line of the coxal axis that it can have little effect as a motor of the coxa. 
The mesal promotor and the mesal remotor take their origins on the metathoracic basal components of the pterothoracic endosternum (fig. $24 \mathrm{~A}, 104,106)$; the lateral promotor (IO3) arises on the ridge between the metapleuron and the propodeum.

The coxo-trochanteral joint.-The union between the coxa and the trochanter in each leg of the bee is a dicondylic hinge with an approximately horizontal anteroposterior axis. The movements of the trochanter on the coxa, therefore, are up and down, and are best expressed as levation and depression. In the bee the trochanter of each leg is provided with two levator muscles, one anterior, the other posterior, arising within the coxa, and with two or three depressors having a common point of insertion, but arising both in the coxa and within the thorax. The two levators are inserted directly on the trochanter or close to it; the fibers of the depressor muscles have no direct connection with the trochanter, but are inserted on a large depressor apodeme arising from a small plate in the ventral articular membrane at the base of the trochanter. The trochanteral muscles are the motors of the entire part of the leg (telopodite) beyond the coxa, the movements of which are in a plane at right angles to the coxal plane of movement.

In the fore leg the relatively small anterior and posterior levators of the trochanter (fig. I3 E, H, 59, 60) arising in the coxa are inserted on the trochanteral base just beyond the coxo-trochanteral articulations (E, $f, g$ ). The depressors include a long thoracic muscle $(\mathrm{E}, \mathrm{H}, 6 I$ ) arising on the prothoracic pleural apophysis (figs. I I C, I2 C, $6 I$ ), which is inserted on the end of the depressor apodeme of the trochanter (fig. I3 E, H), and a large group of fibers (62), arising on the mesal wall of the coxa, inserted on the sides of the apodeme and on the apodemal plate. Since the articular surfaces of the trochanter $(E, f, g$ ) are much closer to the insertions of the levator muscles than to the base of the depressor apodeme, the effectiveness of the large depressor muscles is greatly increased by their advantageous leverage.

In the mesothoracic legs the two levator muscles of each trochanter arise on the lower (lateral) wall of the coxa (fig. $22 \mathrm{D}, 84,85$ ), and are inserted anteriorly and posteriorly on the base of the trochanter. The thoracic muscle of the depressor group (D, G, 86) takes its origin on the side of the endosternum (C); the coxal fibers are distinctly divided into two muscles ( $D, G, 87,88$ ), one anterior, the other posterior, separated by the pedal nerve $(G, N v)$ entering between them into the trochanter. 
The trochanter of each hind leg has two large levator muscles (fig. 24 C, D, I07, I08), a long thoracic depressor (C, E, I09) arising on the metathoracic arm of the endosternum (A, I09), and a single, broad coxal depressor $(\mathrm{C}, \mathrm{E}, I I O)$. The levators $(I 07, I 08)$ are inserted in the dorsal articular membrane (D), the depressors ( 100 , $I I O)$ on a long ventral apodeme $(A p)$.

The trochantero-femoral joint.-The hinge between the trochanter and the femur differs from all the other leg joints in that its axis traverses the vertical plane of the leg, and is, moreover, strongly oblique, the dorsal end being distal to the ventral end (figs. I3 $\mathrm{H}$, ${ }_{24} \mathrm{C}$ ). The movements of the femur on the trochanter, therefore, are those of production and reduction, but, owing to the obliquity of the hinge, the femur acquires also an upward slant as it turns backward. A single reductor muscle activates the femur.

The obliquity of the trochantero-femoral joint in the vertical plane of the leg gives the trochanter a firm pressure or hold on the femur in responding to the depressor and levator action of the trochanteral muscles, the trochanter and the femur being thus moved as a unit on the coxo-trochanteral articulation.

The character of the trochantero-femoral joint differs but little in the three legs of the bee. In each leg there is anteriorly a narrow hinge line between the two adjoining segments (figs. $13 \mathrm{H}, 24 \mathrm{C}$ ), with an articular point $(h)$ at the upper end; only in the hind leg is there a definite ventral articulation (fig. $24 \mathrm{C}, \mathrm{F}, i$ ). Posteriorly the trochanter and femur are separated by a wide, thick, strongly elastic conjunctiva (fig. $\mathrm{I} 3 \mathrm{~B}, c n j$ ). The femoral reductor muscle (fig. $\mathrm{I} 3 \mathrm{H}$, 63; fig. $22 \mathrm{D}, \mathrm{G}, 89$; fig. $24 \mathrm{C}, I I I$ ) arises anteriorly by a broad base in the trochanter and its convergent fibers are inserted on a shelflike apodemal plate projecting from the posterior rim of the basal foramen of the femur (figs. I3 F, 24 F, $A p$ ). The contraction of the muscle, therefore, turns the femur posteriorly on the anterior hinge with the trochanter; the muscle is opposed by the resistence of the elastic posterior conjunctiva, which restores the femur to its original position on relaxation of the reductor muscle.

The femoro-tibial joint.-The "knee" joint of each leg has strictly a hinge motion on a horizontal axis between anterior and posterior articulations. The movements of the tibia, therefore, are those of extension and flexion on the end of the femur; they are produced by dorsal and ventral muscles lying in the femur. The head of the tibia is somewhat bent toward the femur, and the hard wall of the femur is deeply emarginate below the joint, allowing the tibia to be flexed close against the femur. 

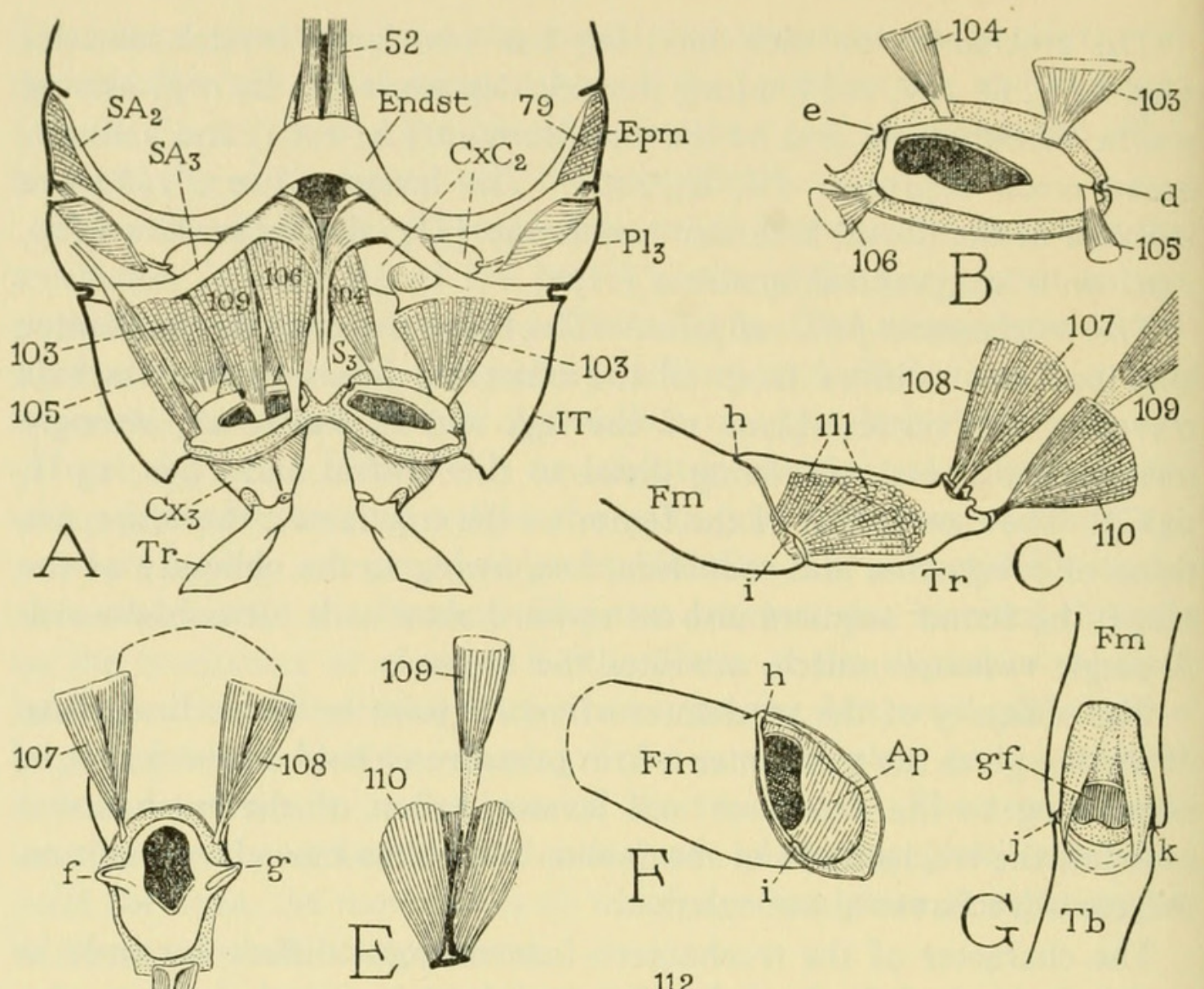

112

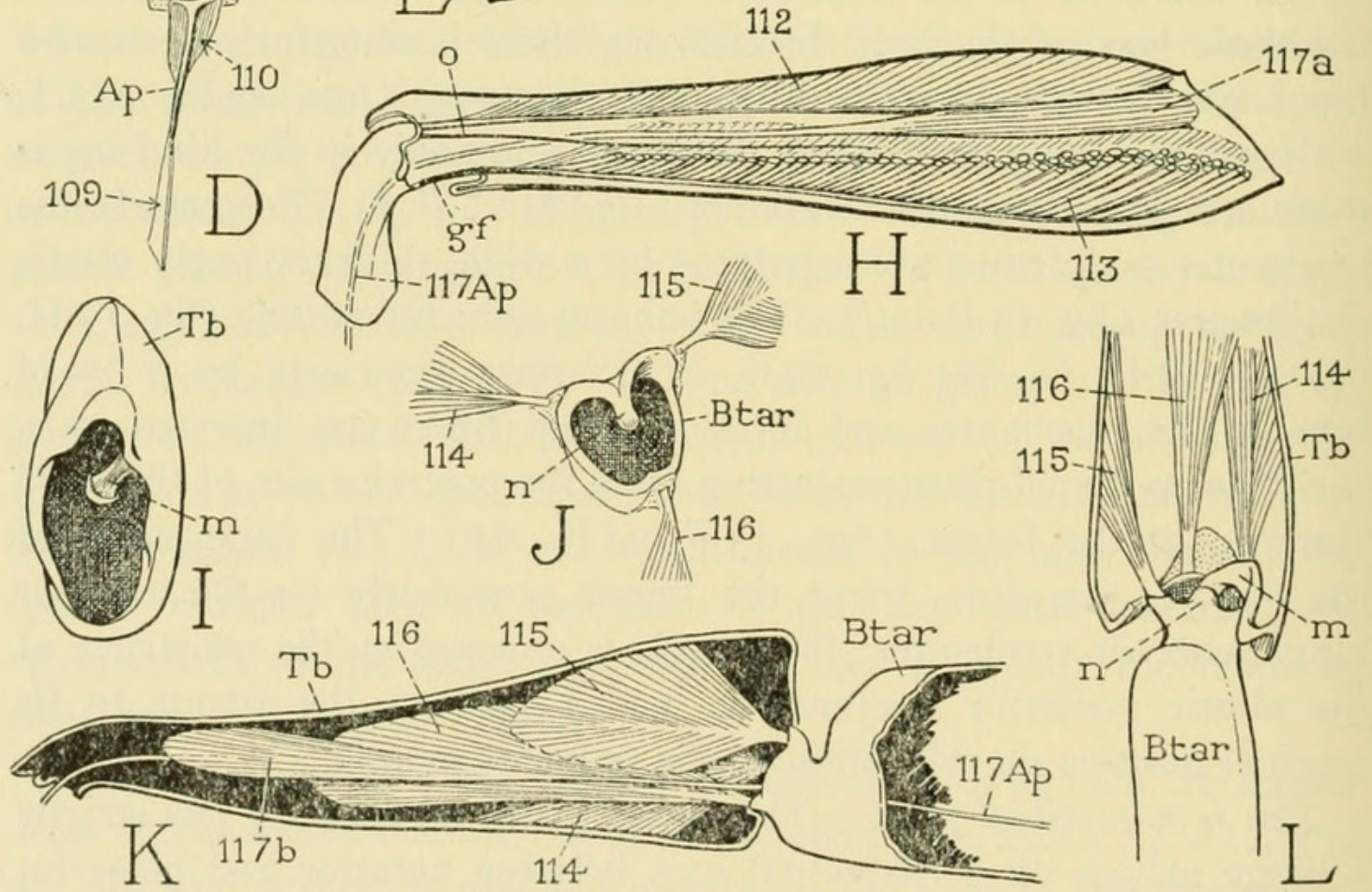

FIG. 24.-The metathoracic leg, muscles and joints (drone).

$A$, endosternum and ventral wall of posterior part of pterothorax, showing muscles of metathoracic coxae, dorsal view. B, base of right hind coxa and muscle insertions, dorsal view. $\mathrm{C}$, right trochanter and base of femur, with trochanteral and femoral muscles, anterior (outer) view. D, proximal view of right trochanter and muscle insertions. E, depressor muscles of trochanter. F, base of right femur, anterior view. G, femoro-tibial joint of right hind leg, ventral view, $H$, right hind femur with muscles, anterior view. I, distal end of right tibia. J, proximal end of right basitarsus. $\mathrm{K}$, right hind tibia and muscles, posterior view. L, tibiotarsal joint of right hind leg, dorsal view.

$d-e$, pleurosternal axis of coxa; $f, g$, anterior and posterior coxal articular processes of trochanter; $h, i$, dorsal and ventral trochantero-femoral articulations; $j, k$, anterior and posterior femoro-tibial articulations; $m$, tibial articular process of tibio-tarsal joint; $n$, tarsal articular process of tibio-tarsal joint; $o$, ligament of femoral chordotonal organ. 
The femoro-tibial joint differs but little in the three legs of the bee. Its mechanism is well exemplified in the middle leg, which, seen from below (fig. $22 \mathrm{~J}$ ), shows the two articular points $(j, k)$ of the joint and the deep membranous ventral emargination of the wall of the femur. The articulations consist of two strong basal lobes of the tibia (L), which fit against corresponding processes concealed within the end of the femur $(\mathrm{K}, j, k)$. The conjunctival membrane below the joint $(\mathrm{H}, \mathrm{J})$ contains a small, partly invaginated genuflexor plate $(g f)$, to the apex of which is attached the tendon of the broad, pinnate flexor muscle of the tibia ( $9 I)$ lying ventrally in the femur. The fibers of the extensor muscle arise along the dorsal wall of the femur $(\mathrm{H}, 90)$, and are inserted on an axial tendon attached to the recurved dorsal head of the tibia $(\mathrm{H}, \mathrm{L})$. In the hind leg the joint structures are essentially the same as in the middle leg, though somewhat different in shape (fig. $24 \mathrm{G}$ ), and the tibial muscles are larger $(\mathrm{H}, I I 2, I I 3)$.

The femur of each leg contains in addition to the tibial muscles a branch of the flexor muscle of the pretarsal claws (fig. $24 \mathrm{H}, I I 7 a$ ), which is inserted on the end of a long tendon ( $I I 7 A p)$ extending through the tibia and tarsus. Finally, there is to be found in each femur a slender, tendinous thread $(o)$ attached on the head of the tibia just anterior to the extensor tendon (fig. $22 \mathrm{~L}$ ), which extends proximally in the femur, and probably belongs to a chordotonal organ such as occurs in the femur of various other insects.

The tibio-tarsal joint.-The joint between the tibia and the tarsus in each leg of the bee is quite different from that between any of the other leg segments; it is monocondylic, and the tarsus is movable on the tibia by three muscles. The single point of articulation is median and dorsal, but is concealed within the distal end of the tibia; two of the muscles are inserted respectively anterior and posterior to the articulation, the third muscle is ventral.

The essential features of the tibio-tarsal joint are the same in all three legs, but structural details and the mechanism are somewhat different in the hind legs. In the first and the second leg the proximal end of the long basitarsus presents dorsally three knobs (fig. $22 \mathrm{E}$ ). The middle knob $(n)$ is the articular condyle; it is received on the concave surface of a large, hook-shaped articular process $(m)$ that descends from the dorsal wall inside the end of the tibia $(\mathrm{I}, \mathrm{m})$. The lateral knobs give attachment to the anterior and posterior tibial muscles (E, 92, 93), which arise on the corresponding walls of the tibia. In the fore leg and the middle leg the attachments of these muscles on the tibia are so nearly on a level with the articular condyle 
that the anterior muscle (92) acts as a productor of the tarsus, and the posterior muscle (93) as a reductor when tension is exerted on the muscles in a dead specimen. Morison (I927) calls these two muscles anterior and posterior "extensors" of the tarsus, but if they have an extensor effect on the tarsus, the action is weak. The third tarsal muscle arises on the lower wall of the tibia (fig. $22 \mathrm{~F}$, I, 94) and is attached by an axial tendon in the ventral conjunctival membrane of the joint. This muscle unquestionably is a depressor of the tarsus. The opposite movement of the tarsus, if not produced by the lateral muscles, must result from the elasticity of the articular conjunctiva. In the fore leg the tibio-tarsal joint mechanism operates the antenna cleaner.

The tibio-tarsal joint of the hind leg, as above mentioned, differs in some ways from that of the other legs. The articular condyle of the basitarsus is a long, curved arm descending from the dorsal rim of the proximal tarsal foramen (fig. $24 \mathrm{~J}, n$ ) ; the corresponding articular process of the tibia $(I, m)$ is a strong sclerotization of the anterior wall of the tarsal socket in the end of the tibia $(L, m)$, which curves inward over the tendon of the anterior muscle (II4), dips downward, and turns distally to articulate ventrally with the lower end of the tarsal condyle $(n)$. The articulation of the two segments is thus almost at the center of the tarsal foramen. As a consequence, the attachment of the posterior tarsal muscle ( J, K, L, II5) is so far above the level of the articulation that this muscle, representing the tarsal reductor in the other legs, becomes in the hind leg a strong levator of the tarsus. The anterior muscle (II4), being inserted nearly opposite the articular point, is evidently a productor; the ventral muscle (II6) is the tarsal depressor.

The structure of the tibio-tarsal joint of the hind leg is the same in both the drone and the worker, and hence has no direct relation to the pollen press of the latter. The mechanism of the joint in the hind leg, however, especially the combined levator-productor action of the two lateral muscles, is unquestionably conducive to the effective action of the press.

The tarsal joints.-The narrowed base of each of the small tarsal subsegments, or tarsomeres, beyond the large basitarsus fits into a receptive cavity in the end of the preceding tarsomere (fig. $23 \mathrm{G}$ ), and each of these joints is monocondylic, as is the tibio-tarsal joint itself. The dorsal lip of the minute basal foramen of each small tarsomere is drawn out into a long articular process, which fits into a socket formed by an inward extension of the lip of the distal foramen of the tarsomere preceding. There are no intratarsal muscles giving 
individual movement to the tarsomeres, but the entire tarsus is traversed by the flexor-muscle tendon $(I I 7 A p)$ of the pretarsal claws, and a pull on this tendon causes a deflexion of the slender part of the tarsus beyond the basitarsus.

The pretarsus.-The pretarsus of the honey bee has a complex and highly specialized structure (fig. $25 \mathrm{~A}, \mathrm{~B}$ ). The median part of the segment is differentiated into a conical, stalklike basal part (E) from which the claws arise laterally, and an oval distal lobe, which is the arolium $(A r)$. In the ordinary position of the foot (E) the arolium stands upward between the claws on the end of the basal support. Dorsally the arolium presents a deep concavity (A) between its upturned lateral parts, and in its outer wall is a dark, elastic, $\mathrm{U}$-shaped band, the arcus (B, E, arc), which embraces the base of the arolium ventrally with its arms extending distally in the lateral walls. The conical base of the pretarsus contains dorsally an elongate median sclerite (A, E, $m n$ ) armed with five or six long, thick, curved spines. By its widened base the sclerite is articulated to the end of the tarsus between the bases of the claws, and its narrowed distal end is attached like a handle to the base of the scoop-shaped arolium $(\mathrm{H})$, for which reason the sclerite may be termed the aroliar mamubrium. In the ventral wall of the pretarsal base is a broad, weakly sclerotized plate, the planta $(\mathrm{B}, \mathrm{E}, \mathrm{pln})$, thickly beset with strong spines diverging distally.

The claws are hollow, strongly sclerotized lateral outgrowths of the membranous lateral walls of the pretarsal base. Each claw has two points of unequal length, and in size and shape the claws differ considerably as between the drone (fig. $25 \mathrm{~A}, \mathrm{~B}$ ) and the worker (C, D). Dorsally the claws are articulated individually to two small knobs on the end of the tarsus $(\mathrm{A}, o, p)$, corresponding with the articular condyles at other dicondylic joints of the leg, but the claws themselves are devoid of muscles.

The motor apparatus both of the claws and of the arolium consists of the depressor muscle of the pretarsus, and of accessory sclerites in the ventral articular membrane at the base of the segment. The principal one of the basal sclerites is a large median plate, known as the unguitractor plate (fig. $25 \mathrm{~B}, U t r$ ), which is comparable with the genuflexor plate below the knee joint. In the membrane at the proximal end of the unguitractor plate is attached the tendon of the pretarsal muscle $(95 A p)$, and at the distal angles are two small auxiliary sclerites (aux) intermediate between the unguitractor plate and the bases of the claws. The pretarsal tendon extends forward through the tarsus (fig. $23 \mathrm{G}$ ) into the tibia (fig. $24 \mathrm{~K}$ ), where it 
divides into two branches, one branch giving insertion to a group of fibers arising posteriorly in the tibia $(I I>b)$, the other, going on into the femur, to a second group $(\mathrm{H}, I I 7 a)$ arising posteriorly in
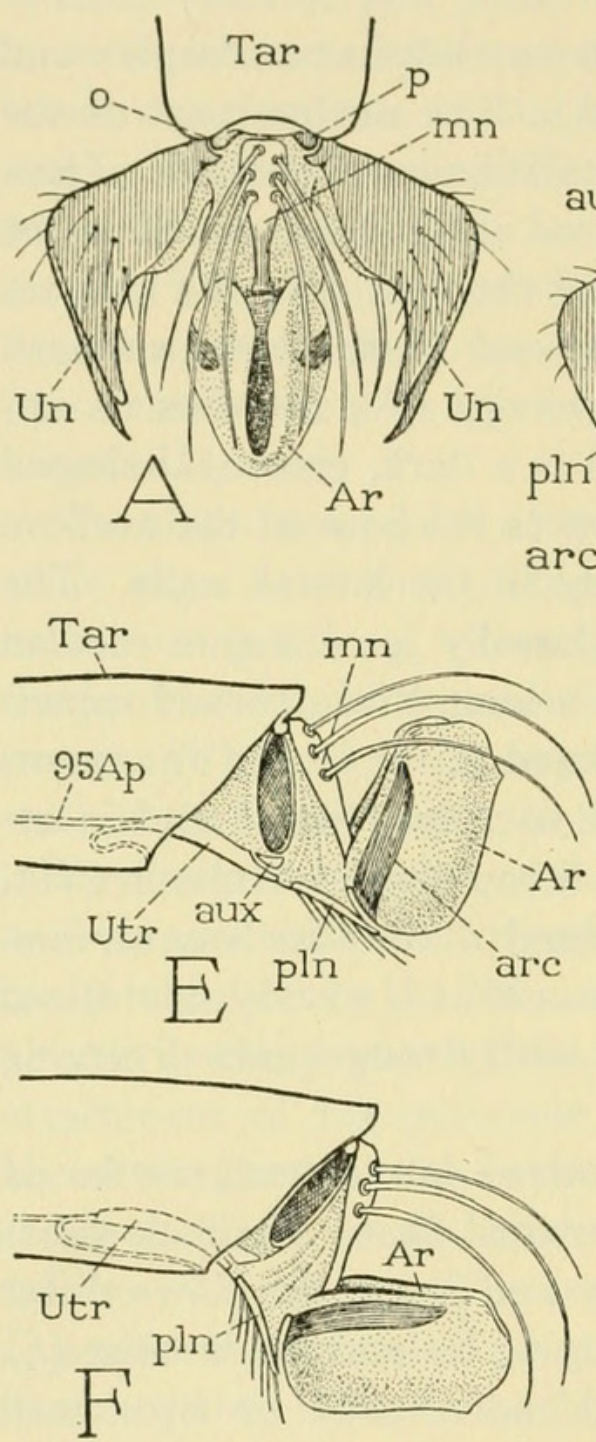
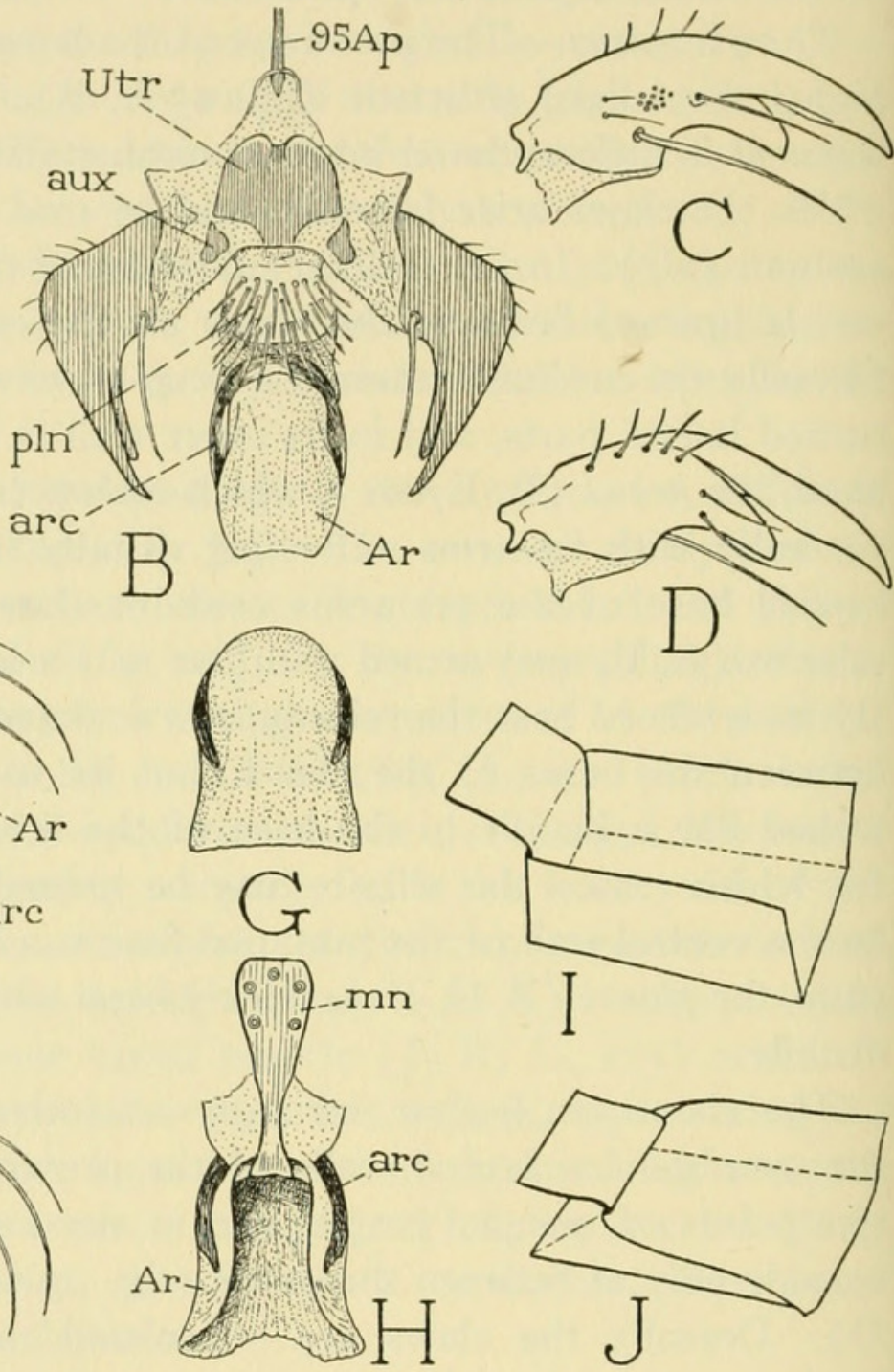

FIG. 25.-The pretarsus.

A, pretarsus of middle leg of drone, dorsal surface, flattened, inner sides of claws uppermost, arolium turned upward. B, same, ventral surface, arolium depressed and spread out. C, posterior claw of middle leg of worker, outer surface. D, anterior claw of hind leg of worker, inner surface. E, pretarsus and end of tarsus, claws removed, arolium turned upward, semidiagrammatic. F, same, arolium depressed. G, ventral surface of fully depressed and flattened arolium. H, arolium and manubrium, dorsal view. I, paper model of arolium and manubrium in nonfunctional condition. J, same, illustrating flattening of arolium by downward pressure on manubrium.

$95 \mathrm{Ap}$, tendonlike apodeme of muscle of pretarsus (retractor of the claws); $A r$, arolium; arc, arcus; aux, auxilia; $m n$, manubrium; $o, p$, anterior and posterior tarso-pretarsal articulations; pln, planta; Tar, end of fifth tarsomere; Un, claw (unguis); Utr, unguitractor plate.

the base of the femur. The tension of the pretarsal muscle on the unguitractor plate is distributed to the claws through the auxiliary sclerites, and thus flexes the claws, for which reason the muscle is 
known as the flexor of the clarws. The direct effect of the muscle pull, however, is exerted on the base of the pretarsus, and, unless the claws interfere, it flexes the pretarsus on the end of the tarsus (fig. $25 \mathrm{~F}$ ) and brings the arolium into a horizontal position.

The arolium is an organ that enables the bee to cling to smooth surfaces on which the claws cannot hold. When the arolium is in use as an adhesive organ the claws are turned proximally with their points directed outward, and the arolium is horizontally extended, its lateral walls spread out flat, its under surface (fig. $25 \mathrm{G}$ ) pressed against the support. The extension and flattening of the arolium has been explained by Arnhart (I923; see Snodgrass, I925) as caused by a secretion liquid forced into the foot from a gland in the end of the tarsus; but a study of the mechanism of the pretarsus shows that the action of the arolium including its changes in shape can be produced entirely by mechanical means.

With a live bee it may be observed that when the flexing claws encounter on a smooth surface nothing to restrain them or to give them a hold, they turn helplessly upward beneath the end of the tarsus with their points sprawling outward. The tension of the contracting pretarsal muscle is then exerted on the base of the pretarsus, which turns abruptly downward bringing the arolium into a horizontal position, after which the arolium extends, spreads out flat, and clings to the support. If the foot of a dead bee is in the position shown at $\mathrm{F}$ of figure 25 , an upward pressure on the retracted planta $(p l n)$ extends the pretarsus until the planta comes into the same horizontal plane as the arolium $(A r)$, and now, with continued pressure, the arolium itself automatically unfolds and spreads out with its flattened under surface downward. This action of the arolium can be induced by manipulation of a detached pretarsus, in which there can be no possibility of liquid pressure from within the tarsus. The same kind of action, moreover, can be illustrated with a piece of paper cut and folded into the form of a scoop (I) ; a vertical compression of the base of the scoop spreads the sides widely apart $(\mathrm{J})$, giving thus a close imitation of the partly expanded arolium $(\mathrm{H})$.

The similar response of the arolium to pressure against the planta is evidently caused in the living bee by the downward pressure of the tarsus, which flattens the pretarsus and compresses the arolium against the support beneath it. The manubrial sclerite of the pretarsus (fig. $25 \mathrm{E}, \mathrm{H}, m n$ ), however, representing the handle of the paper scoop $(I, J)$, plays an important part in the expansion of the arolium. With the extension of the foot, the manubrium turns backward on the end of the tarsus and hence pushes on the upper edge of the aroliar 
base, thus augmenting the effect of the pressure on the planta; finally, when fully extended, the manubrium clamps down on the arolium and holds it securely in the spread condition. There can be little doubt, therefore, that the entire action of the arolium is a mechanical result of the pull of the pretarsal muscle in conjunction with pressure of the foot against the support when the claws fail to take a hold on the latter. It has been said that the arolium adheres to smooth surfaces by means of a sticky exudation from the spines of the planta, but observation fails to reveal the presence of any such adhesive. The upper surface of the arolium is covered with minute hairs, but its under surface is almost entirely bare.

\section{THE ABDOMINAL PETIOLE}

The outstanding feature in the external organization of the clistogastrous Hymenoptera is the secondary consolidation of the fourth body segment with the thorax, and the division of the body by a deep constriction between the fourth and fifth segments, accompanied by a narrowing and usually an elongation of the anterior end of the fifth segment, or even of the entire segment, to form a petiole. Two important mechanical advantages result from these alterations, which take place during the pupal stage of development. First is the greatly increased length that the dorsal wing muscles of the thorax are able to acquire by a posterior extension of the mesothoracic postphragma into the fourth segment (fig. I6 C) ; second is the high degree of potential mobility conferred on the shortened abdomen. The first advantage is self-evident, the second needs elucidation.

In the more generalized insects the abdomen is broadly attached to the thorax by its first segment, and the muscles that move the abdomen as a whole are dorsal and ventral intersegmental muscles between the metathorax and the abdomen. In winged insects, however, the dorsal intersegmental muscles of the metathorax become functionally a part of the flight mechanism (fig. $26 \mathrm{~A}, d_{3}$ ) by a transfer of the ridge or phragma of their abdominal attachment to the thorax. As a consequence, the dorsal metathoracic muscles of winged insects have no motor effect on the abdomen. Ventral muscles between the thorax and the abdomen are seldom strongly developed, and they may be absent; when present they comprise generally one pair or two pairs of slender muscles arising anteriorly on the metasternal apophyses and attached posteriorly sometimes on the first abdominal sternum (A), but more commonly for greater effectiveness on the second (C) or even the third, fourth, or fifth sternal plate. 
Such insects have little mobility of the abdomen on the thorax, even though the two parts of the body may be separated by a constriction.

A comparison of the structural relation between the thorax and the abdomen in clistogastrous Hymenoptera (fig. 26 B) with that in
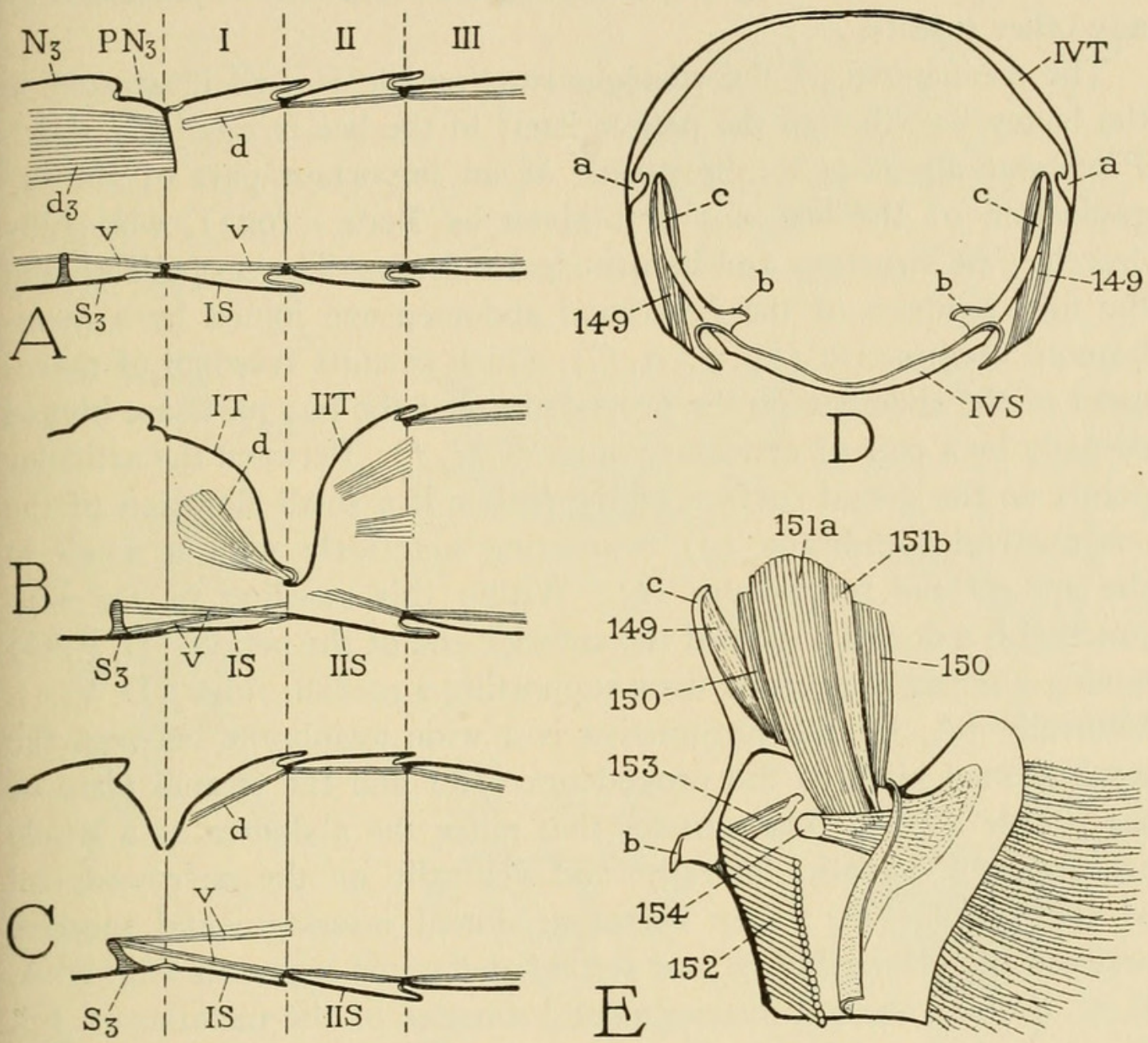

FIG. 26.-Relation between the propodeum and the abdominal petiole; lateral muscles of an abdominal segment.

A, diagram of thoracico-abdominal relation in a generalized winged insect: the dorsal intersegmental muscles of metathorax $\left(d_{3}\right)$ are wing motors and do not move the abdomen. B, specialized condition in clistogastrous Hymenoptera: dorsal and ventral muscles $(d, v)$ of first abdominal segment (propodeum) become effective motors of rest of abdomen. $\mathrm{C}$, usual structure in higher winged insects: abdomen but little movable on thorax. D, anterior endview of abdominal segment $I V$ of drone, showing mechanism of dorsoventral expansion by contraction of reversed anterior lateral muscles. E, right half of abdominal sternum $I V$ of drone, with sternal and lateral muscles.

$a$, anterior tergal apodeme; $b$, anterior sternal apodeme; $c$, lateral sternal apodeme; $d$, dorsal intersegmental muscles; $v$, ventral intersegmental muscles.

either a generalized winged insect (A) or a more specialized form (C) will show clearly the advantage derived by the Clistogastra from having the first abdominal segment solidly joined to the thorax, and a constricted point of movement established between the first and 
second segments of the abdomen. The modification allows the muscles of the transposed first abdominal segment $(\mathrm{B}, d, v)$ to become effective motors of the free part of the abdomen. The aculeate Hymenoptera thereby acquire a much greater abdominal mobility for the use of the ovipositor or sting or for mating activities than is possessed by any other insects.

The mechanism of the clistogastrous petiole is well illustrated in the honey bee, though the petiole itself in the bee is relatively short. Particular attention to the petiole as an important part of the organization of the bee was first given by Betts (I923), who fully describes its structure and its principal muscles. The propodeum and the first segment of the functional abdomen are joined by a membranous conjunctiva (fig. $27 \mathrm{~A}, \mathrm{C}$ ), which permits freedom of movement of the abdomen on the propodeum, but the two parts are hinged dorsally by a pair of articular points $(C, f, f)$. Between the articular points on the dorsal surface of the petiole is a small elevation of the conjunctival membrane $(e)$ connecting anteriorly with a notch in the end of the propodeum $(t)$. Within this swelling of the conjunctiva is a dorsal pocket of the anterior end of the petiole $(D, F, G)$ having a strongly sclerotic floor supporting a median ridge $(\mathrm{D}, \mathrm{F}, r)$. Ventrally (A, E) the conjunctiva is a wide membrane between the weak sternal plate of the propodeum $(I S)$ and the sternal plate of the petiole $(I I S)$. The muscles that move the abdomen as a whole are attached dorsally, laterally, and ventrally on the narrowed end of the petiole $(G)$; they represent dorsal intersegmental muscles between the primarily first and second abdominal segments (fig. $26 \mathrm{~A}$, $\mathrm{B}, \mathrm{C}, d)$, and ventral intersegmental muscles of the metathorax (B, $\mathrm{C}, v)$ that pass over the first abdominal sternum to attach on the second.

The petiole muscles of the honey bee include two pairs of dorsal muscles and two pairs of ventral muscles, but on account of the positions of their attachment on the petiole, one pair of the dorsal muscles are levators of the abdomen, one pair of ventrals are depressors, and the other two pairs evidently produce sidewise movements. The levator muscles arise by broad, spreading bases on the lateral walls of the propodeum (figs. $27 \mathrm{~B}, \mathrm{C}, 28 \mathrm{~A}, 120$ ), and are inserted by strong tendons that converge to their attachments on the posterior end of the ridge on the floor of the petiole pocket (fig. $27 \mathrm{D}$, F, G). These muscles thus acquire a short leverage on the propodeal fulcra $(f)$. The depressors of the abdomen are a pair of long ventral muscles arising on the metathoracic components of the pterothoracic endosternum (fig. $27 \mathrm{E}, I I 8$ ), and inserted medially on the 
anterior margin of the sternal plate of the petiole (E, G). The lateral muscles of the petiole include a pair of two-branched dorsal muscles arising anteriorly on the sides of the propodeum (figs. $27 \mathrm{~B}, 28 \mathrm{~A}$, I2I) and inserted on the lateral angles of the tergum of the petiole
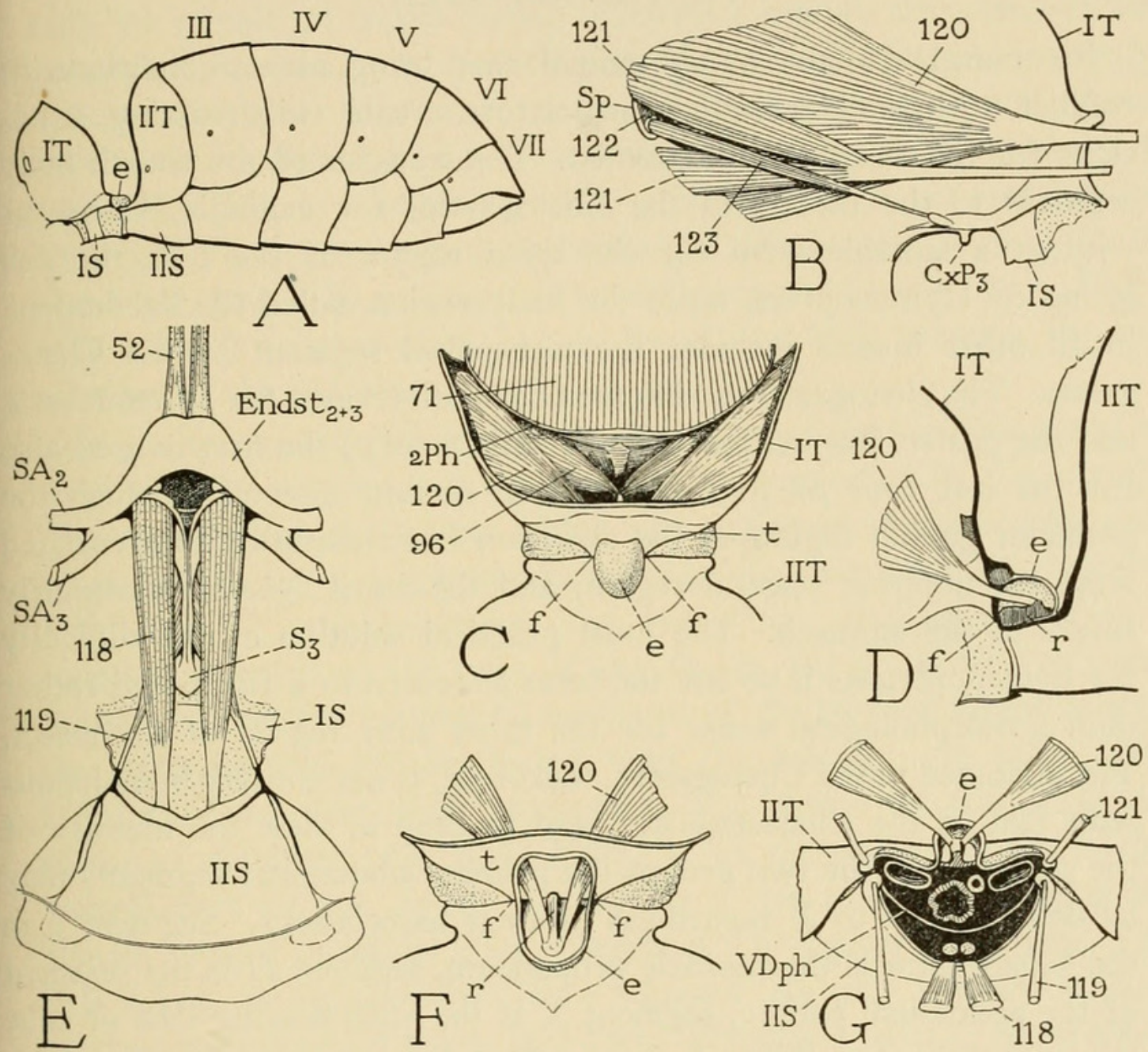

FIG. 27.-The propodeum and petiole. (Figures, except E, from Snodgrass, I933.)

A, abdomen of worker, including propodeum. B, muscles in right side of propodeum, mesal view. C, propodeum and petiole, dorsal wall of propodeum removed, exposing muscles. D, levator mechanism of abdomen exposed by removal of left wall of propodeum and petiole. E, ventral muscles of petiole, dorsal view. F, petiole and end of propodeum, dorsal view, upper wall of petiolar pocket (e) removed to show insertion of levator muscles (I20). $\mathrm{G}$, anterior end of petiole, showing muscle attachments on tergal and sternal plates.

$e$, membranous roof of dorsal pocket of petiole; $f, f$, fulcra of movement of abdomen on propodeum; $r$, median ridge on floor of petiolar pocket; $t$, posterior area of propodeum adjoining petiole.

(fig. $27 \mathrm{G}$ ), and a pair of ventral muscles arising on the metathoracic sternum (E, IIO), which are inserted laterally on the end of the petiole sternum $(\mathrm{E}, \mathrm{G})$. The cavity of the neck of the petiole is divided transversely by the ventral diaphragm (G,VDph), which 
begins in the metathorax. The larger dorsal channel gives passage to the tracheal trunks, the oesophagus, and the aorta; the ventral channel contains the nerve cords.

\section{THE ABDOMEN}

Structural changes in an animal that bring about conditions in conflict with standardized nomenclature create embarrassing situations for the descriptive anatomist. The transfer of the fourth body segment to the thorax in the Clistogastra, for example, leaves us without a suitable term for the third region of the body in this group of Hymenoptera, since the body region called the "abdomen" in all other insects includes the transposed segment of the Clistogastra. The clistogastrous abdomen has been termed the postabdomen, and the gaster, but neither name is satisfactory; the first is used also for the last four or five segments in certain insects in which the posterior genital region of the abdomen is structurally differentiated from the anterior visceral region, and the word "gaster" ordinarily refers to the stomach. The most practical solution of the difficulty for hymenopterists is to use the term abdomen in a functional rather than a morphological sense for the third body region of the insect. The abdomen in the Clistogastra, therefore, is not entirely homologous with that of the Chalastogastra, and to keep in view the identity of the segments in the two groups the primary abdominal segments may be designated $I$ to $X$ regardless of their associations. Segment $I$ in the Clistogastra is the thoracic propodeum, segment $I I$ is the segment of the abdominal petiole, segment $X$ is the anus-bearing lobe or tube known as the proctiger. In many Hymenoptera the true anus lies within a terminal invagination of the proctiger, which possibly represents segment $X I$.

General structure of the abdomen.-The segments of the pregenital region of the abdomen in the honey bee have well-developed tergal and sternal plates, but there are no lateral, or "pleural," sclerites, and the spiracles lie in the sides of the terga (fig. $27 \mathrm{~A}$ ). The tergal plates, except the first, are provided each with a large anterior tergal apodeme on each side (figs. $26 \mathrm{D}, 28 \mathrm{~A}, 29 \mathrm{~A}, a$ ) ; the sternal plates have each a pair of anterior sternal apodemes (figs. $26 \mathrm{D}, 28 \mathrm{~A}, \mathrm{~B}$, $29 \mathrm{~A}, b)$, and on each side a long lateral sternal apodeme $(c)$. The sternal plates of segments $I I I$ to $V I$ widely underlap each the sternum of the following segment, forming pockets which in the worker conceal the areas of the wax glands on sterna $I V$ to $V I I$. 
In the male bee the dorsum of the exposed part of the abdomen ends with the declivous tergal plate of segment VIII (fig. 29 A), but the sternum of this segment (VIIIS) is concealed within that of segment $V I I$, and the visible part of the venter terminates with sternum $I X$. The tergum of segment $I X$, however, is represented only by a pair of small dorsal sclerites lying at the sides of the proctiger (figs. $29 \mathrm{~A}, 30 \mathrm{~A}, I X T$ ), each of which supports internally a large apodeme (fig. $29 \mathrm{~A}, a p$ ) giving attachment to the dorsal muscles of its segment (B). The sternum of segment $I X$ (figs. $29 \mathrm{~A}$, $30 \mathrm{~A}, \mathrm{~B}, I X S)$ is a relatively large crescentic plate exposed beyond sternum $V I I$, and bears on its posterior margin the two pairs of phallic plates $(l p, p v)$. The proctiger is a membranous lobe (fig. $30 \mathrm{~A}$, Ptgr) with a small subanal plate $(b)$ in its ventral wall between the two sclerites of the ninth tergum.

In the queen and the worker bee the exposed part of the abdomen ends with the tergum and the sternum of segment VII (fig. $27 \mathrm{~A}$ ). The margins of the apical plates are ordinarily closed upon each other, concealing a large invagination cavity that contains segments $V I I I$ and $I X$, the proctiger, and the sting. Segment VIII is a complete annulus at the base of the cavity, but its walls are membranous except for two lateral remnants of the tergum, which contain the spiracles and are associated with the base of the sting. The tergites of segment $V I I I$, therefore, are known as the spiracular plates of the sting (fig. $28 \mathrm{C}$, VIIIT ). In the posterior wall of the ventral part of the eighth segment is a depression containing the female genital openings (D). Segment $I X$ supports the sting, but it is hardly to be recognized as a segment; its only sclerotized parts are two lateral tergal plates that form virtually a part of the stinging apparatus (fig. 3I A, $Q d$ ), and are hence called, because of their position and shape, the quadrate plates of the sting. The venter of the ninth segment $(I X . V)$ is entirely membranous and, when the sting is retracted, is mostly concealed between the oblong plates $(O b)$ of the basal apparatus of the sting.

The abdominal musculature.-The musculature of the abdomen follows a definite plan, the pattern of which is repeated alike in each of the segments, except for omissions in the first and the last three segments, and the addition of a few special muscles in connection with the genital organs and the sting. The typical segmental musculature is complete in segments $I I I$ to $V I$ in both sexes, but the individual muscles of the male (fig. 29 B) are much larger than those of the female (fig. $28 \mathrm{~A}$ ). The somatic abdominal muscles, exclusive 
of the muscles of the dorsal and ventral diaphragms, may be classed as intertergal dorsal muscles, tergosternal lateral muscles, intersternal ventral muscles, and spiracular muscles. The dorsals and ventrals again include internal and external groups of fibers, and the internal dorsal and ventral muscles comprise median and lateral muscles.

For a study of the abdomnial musculature segment $V$ of the worker may be taken as a representative segment (fig. $28 \mathrm{~A}, V$ ), the larger muscles of the drone (fig. 29 B) being less isolated. On each side of the tergum of segment $V$ two bands of muscle fibers diverge posteriorly to attach on the antecosta $(A c)$ of tergum $V I$. These muscles are the internal dorsals, one median ( ${ }_{555}$ ), the other lateral $\left(I_{56}\right)$. The single short external dorsal ( $\left.I_{57}\right)$ is a reversed muscle, since it arises posteriorly on tergum $V$ and extends forward to its insertion on the anterior apodeme $(a)$ of tergum $V I$. The spiracular muscles include a small occlusor (not seen in the figure) attached at both ends on the spiracle itself, as shown in segment VII (C,I80), and a long, slender dilator (A, I59) arising on the lateral apodeme of the sternum $(c)$. Of the three tergosternal lateral muscles, the first $(I 60)$ is a reversed muscle arising ventrally on the lower part of the tergum and inserted dorsally on the long lateral apodeme of the sternum (fig. $26 \mathrm{D}, 149$ ). The other two lateral muscles cross each other obliquely in the female (fig. $28 \mathrm{~A}$ ) from the tergum to the sternum, the one which is external (IVI) being attached on the sternal margin, the other $(\mathrm{I} 62)$ on the posterolateral upper surface of the sternum. In the male (figs. $26 \mathrm{E}, 29 \mathrm{~B}$ ) the second and third lateral muscles are parallel. The external muscle is a broad band of fibers (fig. $26 \mathrm{E}, I_{50}$ ) attached on the sternal margin; the internal lateral muscle includes two distinct groups of fibers ( $I 5 I a, I 5 I b$ ), the second distinguished by its pale, whitish color, both attached on the upper surface of the posterior part of the sternum. The sternal musculature duplicates the pattern of the tergal musculature, there being a pair of posteriorly divergent internal ventrals on each side (fig. $28 \mathrm{~A}, \mathrm{~B}, I 6_{3}, I 64$ ), and a single reversed external ventral (A, I65) between the overlapping parts of the consecutive sterna.

Functionally the internal dorsal and ventral muscles are contractors of the abdomen, inasmuch as they retract the individual tergal and sternal plates. The external dorsal and ventral muscles of the more posterior segments, on the other hand, are extensors of the abdomen, since their contraction protracts the segmental plates by reducing the overlap between them. Similarly, the reversed muscles of the lateral series are dilators of the abdomen (fig. $26 \mathrm{D}, 149$ ), because their contraction separates the tergum and the sternum of each segment, 

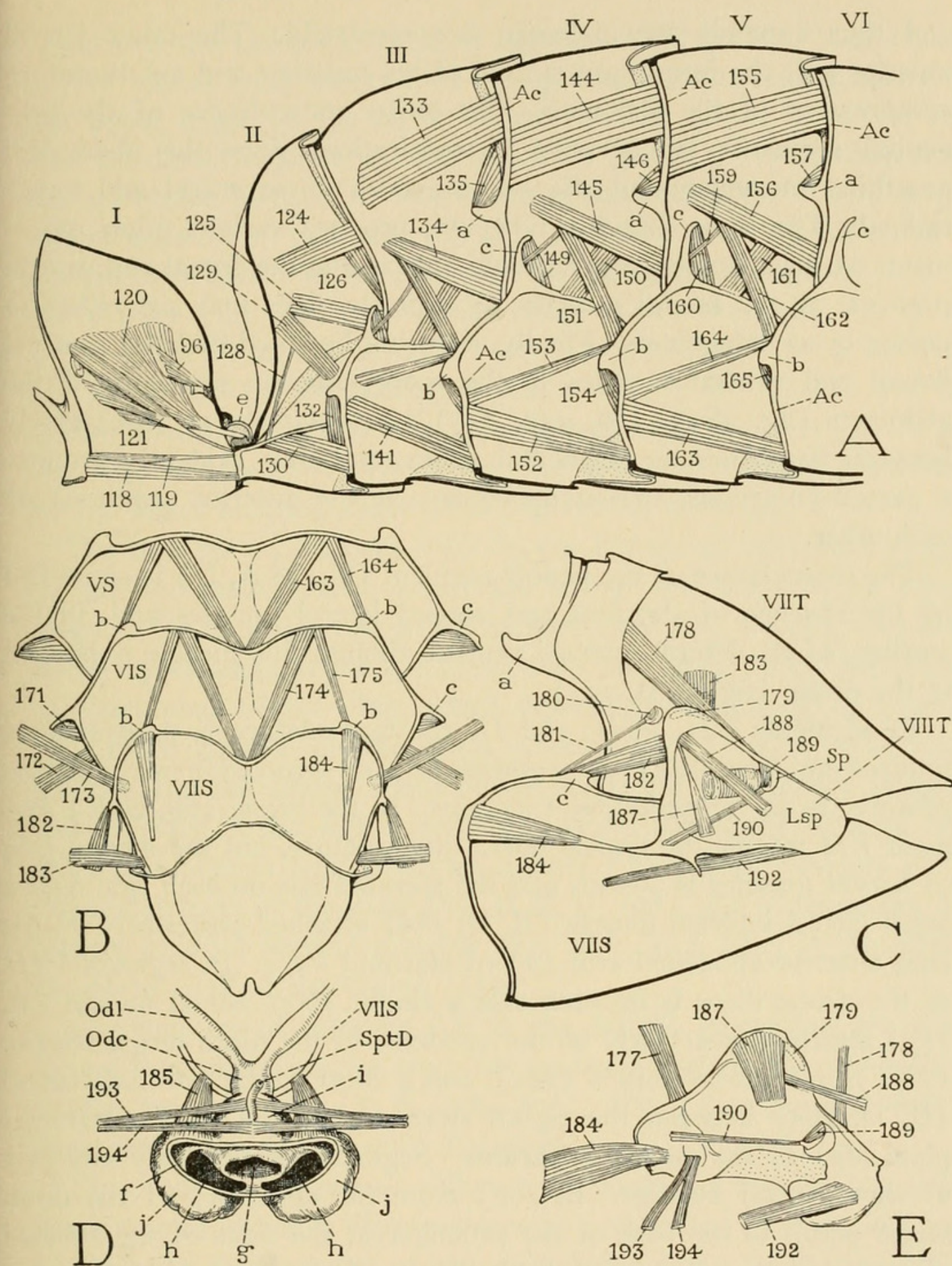

FIG. 28. - The abdominal musculature of the worker and queen. (From Snodgrass, I933, with muscles renumbered.)

A, muscles in right half of anterior part of abdomen of worker, including propodeum, mesal view. $\mathrm{B}$, ventral muscles of abdominal segments $V, V I$, and $V I I$ of worker, dorsal view. C, muscles of right half of segment $V I I$ and of spiracular plate of segment VIII of worker, mesal view. D, genital pouches and oviducts of queen, with attached muscles, posterodorsal view. E, right spiracular plate of segment $V I I I$ of queen, and its muscles, mesal view.

$a$, anterior tergal apodeme; $b$, anterior sternal apodeme; $c$, lateral sternal apodeme; $e$, dorsal pocket of petiole; $f$, edge of shallow external part of genital chamber; $g$, vaginal aperture (opening of median genital pouch) $; h, h$, apertures of lateral genital pouches; $i$, median pouch (vagina) of genital chamber; $j, j$, lateral pouches of genital chamber. 
and thus expands the abdomen dorsoventrally. The other lateral muscles pull the tergal and sternal plates together and are therefore compressors of the abdomen. The entire musculature of the pregenital segments is operative in respiration, since the abdominal breathing movements of the bee are both dorsoventral and longitudinal. The same muscles, however, produce various other movements of the abdomen, and it would appear that the dorsal and ventral muscles, or the lateral muscles of opposite sides may act either in unison or as antagonists. Finally, it should be noted that the external dorsal and ventral muscles in the more anterior segments of the abdomen (fig. $28 \mathrm{~A}, 126,132,135$ ) run obliquely or transversely between the adjoining segmental plates, and thus evidently produce a partial rotary or torsion movement of the anterior segments on each other.

The musculature of the petiole segment (fig. $28 \mathrm{~A}, I I$ ) is simplified by the absence of the first and second lateral muscles and, in the worker, of the lateral internal ventral, though this muscle is present in the drone (fig. $29 \mathrm{~B}, I 3^{I}$ ).

In segments $V I I, V I I I$, and $I X$ the musculature is progressively reduced and altered as these segments become involved in modifications adaptive to the reproductive functions. In the drone (fig. 29 B) segment VII retains the typical dorsal musculature, but the second pair of lateral muscles is absent, and the sternum has on each side only a small lateral internal muscle ( $\mathrm{B}, \mathrm{D}, 186)$ attached posteriorly on the long anterior apodemal arm $(b)$ of sternum VIII. In segment VIII of the drone there is on each side a single, short dorsal muscle (B, 187 ) attached posteriorly on the apodeme of the ninth tergal sclerite $(I X T)$, one lateral muscle $(I 9 I)$, and a short lateral internal ventral (B, D, 195) between the eighth sternum and the small parameral plate $(l p)$ of the genital apparatus. Segment $I X$ has only one pair of short dorsal muscles $(\mathrm{B}, 202)$ from the apodemes of the ninth tergal plates to the base of the proctiger at the sides of the subanal plate, but there are two small phallic muscles on each side (D, 203, 204) arising ventrally on the parameral plate $(l p)$ and attached dorsally on the arm of the penis valve $(p v)$, while finally a long. slender muscle (205) goes from the arm of the penis valve to the mucus gland, to which it is attached just laterad of the termination of the vas deferens.

In the female the musculature as well as the structure of the posterior abdominal segments is very different from that of the male. The tergal musculature of segment VII in the worker includes on each side an internal dorsal (fig. $28 \mathrm{C}, I 78$ ) attached on the upper 
margin of the spiracular plate of segment VIII (VIIIT), and a reversed lateral lying external to the spiracular plate and attached on the upper angle of the latter (I79). In the queen there are two internal dorsal muscles of segment VII (E, I77, I78) attached on the spiracular plate. The lateral musculature consists of two tergosternal muscles on each side ( $\mathrm{C}, I 82, I 83)$, and a very small reversed muscle, not shown in the figures. The sternum of segment VII in the worker (C) has none of the usual ventral muscles, but in the queen a pair of median internal ventrals is present $\left(\mathrm{D}, 18_{5}\right)$ attached posteriorly on the copulatory pouch. In both the worker and the queen, however, there is a large, intersegmental sternotergal muscle (C, I84) arising anteriorly on sternum VII and attached posteriorly on the lower anterior angle of the spiracular plate $(C, E)$.

The muscles of segment VIII in the female all take their origins on the spiracular plates, which are lateral remnants of the eighth tergum. On each side two dorsal muscles (fig. $28 \mathrm{C}, \mathrm{E}, 187, I 88$ ) arise in the upper angle of the spiracular plate and go to the corresponding quadrate plate of the sting, which represents the tergum of segment $I X$. Each spiracle has the usual occlusor (I89) and dilator (I90) muscles, but the dilator arises on the spiracular plate, there being no sternal plate in the eighth segment. From the lower edge of each spiracular plate a long posterior muscle (192) goes forward to the triangular plate of the sting (fig. 3I B, D, I92), and in the queen two slender anterior ventral muscles (fig. $28 \mathrm{E}, 193$, I94) go mesally to the dorsal wall of the vagina (D).

The muscles of segment $I X$ in the female pertain to the sting, except two small muscles from the quadrate plate to the ventral wall of the proctiger (fig. 3I B, 200, 20I). The other muscles will be described in connection with the sting.

The male genital organ.-The phallic organ of the Apidae differs from that of all other Hymenoptera in a reduction of the outer parts and a great development of the endophallic sack. In Apis mellifera the ectophallic parts consist of two pairs of small plates lying behind the last abdominal sternum (fig. $30 \mathrm{~A}, \mathrm{~B}, \mathrm{C}, \mathrm{D}, l p, p v$ ), the small outer pair of which $\left(l_{p}\right)$ may be identified with the parameral plates, and the larger inner pair ( $p v)$ with the aedeagal plates, or penis valves, of other families (see Snodgrass, I94I). Between the penis valves and thick membranous folds uniting them dorsally and ventrally is a widely distensible opening, the phallotreme (Phtr), which leads into the cavity of a huge endophallic invagination (F, Enph) that extends forward as far as the segment of the petiole. 

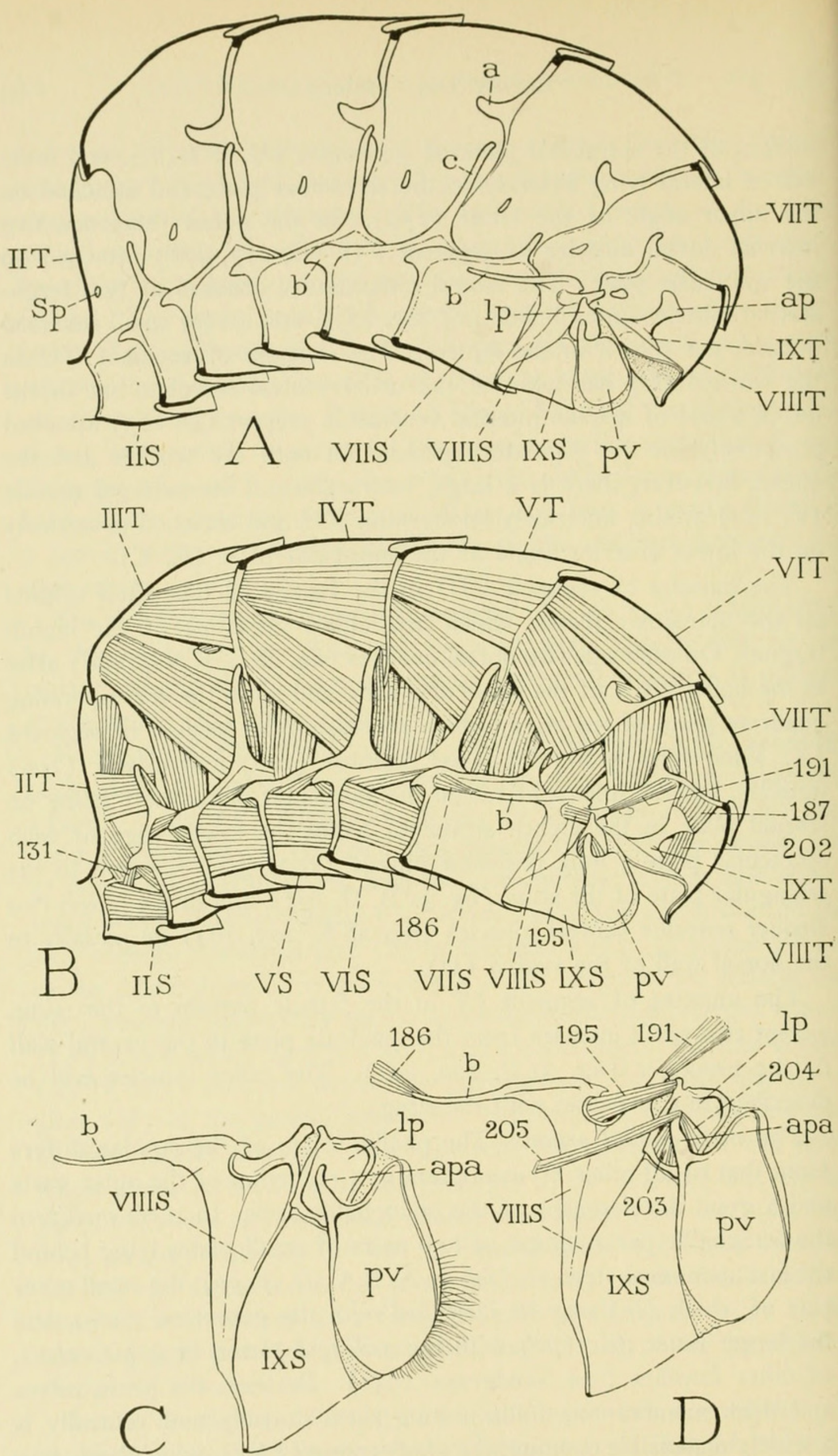

FIG. 29.-The abdominal musculature of the drone, not including the propodeum. (From Snodgrass, 194I, with muscles renumbered.)

A, right half of abdominal wall, mesal view. B, muscles of right half of abdomen, mesal view. C, right halves of sternal plates of abdominal segments $V I I I$ and $I X$, and right phallic

plates, mesal view. D, same, with muscles.
$a$, anterior tergal apodeme; $b$, anterior sternal apodeme; $c$, lateral sternal apodeme. 
The endophallus is the intromittent organ of the bee; during mating it is everted, in part or entirely, and inserted into the genital tract of the queen. Structurally the endophallus is differentiated into three major sections: first, opening directly from the phallotreme, is a large sack, or bursa (fig. 30, F, Brs); the second, middle part is a narrowed neck, or cervix ( $\mathrm{Cer}$ ), connecting the bursa with the third, innermost part, which is a large, thick-walled bulb $(B l b)$. The ejaculatory duct $(D e j)$ opens into the anterior end of the bulb.

The endophallic bursa (fig. $30 \mathrm{~F}, \mathrm{Brs}$ ) has tough membranous walls, which are more or less collapsed and folded in the retracted condition. From the sides of the bursa project a pair of large, tapering, thin-walled, crumpled cornua, or pneumapophyses $(b c)$. In the ventral wall are two somewhat elevated dark areas, the first $(c)$ quadrate in form, the second $(d)$ shield-shaped. Internally these areas are seen to be flat pockets of the bursal wall covered with a dense growth of small, dark spines or thick curved hairs. Elsewhere the inner walls of the bursa are closely dotted with minute spicules, but the linings of the cornua are bare, though wrinkled and finely alveolate in appearance.

The cervix of the endophallus is ordinarily compressed into an irregular shape (fig. $30 \mathrm{~F}, \mathrm{Cer}$ ), but when stretched out straight it is seen to be a wide tube with tough membranous walls. Along the length of its lower wall runs a rounded fold $(e)$ that forms internally a ventral gutterlike channel. Externally the fold is crossed by a series of V-shaped elevations conspicuous by their dark color, which is due to an internal covering of small spines. Similarly, an internal spinecovered patch at the posterior end of the dorsal wall appears externally as a triangular or cordate dark area $(f)$. Near its anterior end the cervix bears dorsally a large diverticulum $(f l b)$, known as the pinnate, or fimbriate, lobe because its margins are deeply cut into flat, overlapping lobules.

The large endophallic bulb (fig. $30 \mathrm{~F}, B l b$ ) has a complex structure, which is not fully understood since it has never been thoroughly studied by histological methods. In the distal half of its dorsal wall are two parallel, elongate, strongly sclerotic plates (E, F, g), which are usually shown in illustrations as external structures, but they are covered by a thin outer tunic that forms a fold in the groove between them and is continued over the rest of the bulb. The plates, therefore, would appear to be sclerotizations of the inner wall of a flat dorsal pouch of the bulb lumen; when the covering tunic is removed the smooth outer surfaces of the plates are exposed (E) and the tapering posterior ends are seen to project freely into the posterior part of 
the cavity of the bulb. The plates are not separable from a large spongy body beneath them that forms the dorsal wall of the bulb cavity and thins out on the sides, where it is continued into a chitinous intima covering the rest of the lumen. From the inner wall of the spongy body two small, posterior triangular plates $(\mathrm{E}, h)$ project laterad of the dorsal plates as free flaps into the cavity of the bulb.

In most drones taken in late summer the endophallic bulb is found to be filled with a dense mass of spermatozoa and fine granular matter, which, in specimens preserved in alcohol, forms a compact body molded into the shape of the interior of the bulb (fig. $30 \mathrm{H}$ ). This body is termed by Zander (I9II) a Samenpatrone ("seminal cartridge," cartouche of Leuenberger, I929). It is not, however, a true spermatophore, or sperm-containing capsule, since it has no retaining wall; its outer surface in preserved specimens is dense and regular, but is minutely pilose because of the great number of sperm tails projecting from it.

The discharge of the spermatozoa is accomplished by eversion of the endophallus. The endophallic walls being entirely nonmuscular, eversion of the organ is brought about naturally by a strong muscular compression of the abdomen, and this function of the abdomen in the male probably accounts for the great size of the abdominal muscles of the drone (fig. 29 B) as compared with those of the queen or worker (fig. 28 A). Artificially a partial evagination of the endophallus is easily produced in a fresh specimen by pressure on the abdomen, and is often seen in killed specimens (fig. $30 \mathrm{G}$ ). Bishop (I920), however, describes methods by which a complete eversion can be induced, and Leuenberger (I929, fig. I7) gives a photograph of the fully everted organ.

In the first stage of the endophallic eversion, or the condition usually produced by artificial compression of the abdomen, the bursa is turned entirely inside out (fig. $30 \mathrm{G}, \mathrm{Brs}$ ) with the everted cornua (bc) projecting upward and outward from its sides. The stretched cervix of the organ now traverses the lumen of the bursa and opens between the bases of the cornua. It is generally supposed (though apparently never observed) that in the copulatory act the cornua, or pneumapophyses, enter the lateral pouches of the female genital chamber (fig. $28 \mathrm{D}, j$ ) ; probably the cervical aperture on the distended bursa is held close to the opening of the median vaginal pouch $(g)$, and the endophallic bulb then everted through the cervix into the vagina. Zander (I9II) contended that the bulb is not everted, since in newly mated queens he found it in the uneverted condition grasped between the base of the ovipositor and the seventh abdominal sternum. On 

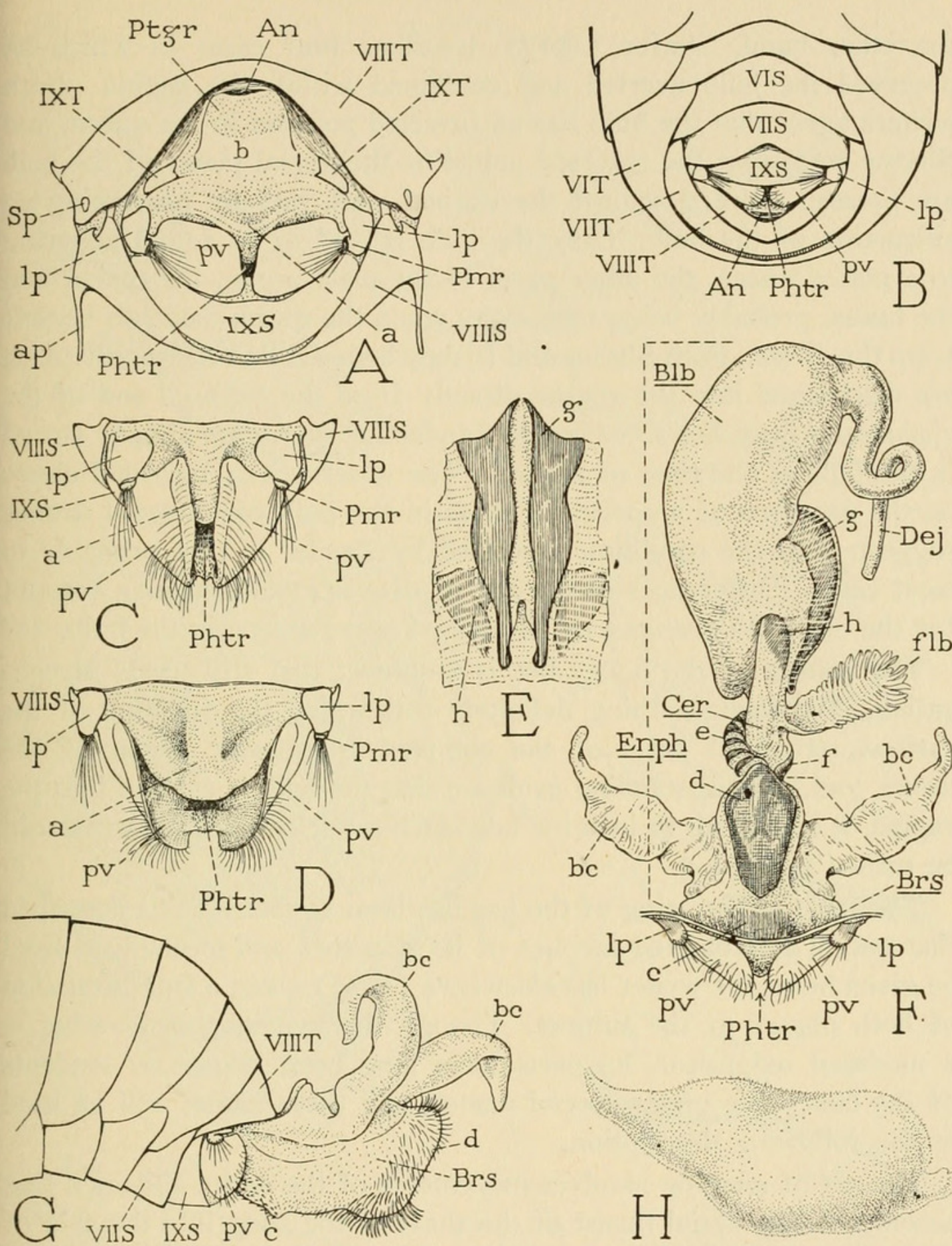

FIG. 30.-The male genitalia. (Figures A to F from Snodgrass, I94I.)

A, segments $V I I I, I X$, and $X$ of male abdomen, end view, showing phallotreme and genital plates between sternum $I X$ and proctiger. $\mathrm{B}$, end of male abdomen, ventral view. C, ectophallus, dorsal view. D, same, phallotreme distended by separation of penis valves $(p v)$. E, dorsal and inner plates of endophallic bulb. F, ectophallus and invaginated endophallus, dorsal view. G, end of abdomen with endophallus partly everted. $\mathrm{H}$, mass of spermatozoa and agglutinating material in bulb of endophallus.

$a$, membranous dorsal lip of phallotreme; $A n$, anus; $a p$, anterior apodeme of sternum $V I I I ; b$, subanal plate of proctiger; $b c$, bursal cornua; $B l b$, bulb of endophallus; Brs, bursa of endophallus; $c$, spiny area of inner bursal wall; $C e r$, neck of endophallus; $d$, setigerous area of inner bursal wall; $D e j$, ductus ejaculatorius; $e$, corrugations of endophallic neck; Enph, endophallus; $f$, setigerous area of inner wall of endophallic neck; $f b l$, fimbriate lobe; $g$, dorsal plate of endophallic bulb; $h$, inner plate of bulb; $l p$, lamina parameralis; Phtr, phallotreme; Pmr, paramere; Ptgr, proctiger; $p v$, penis valve. 
the other hand, Shafer (I9I7) describes four cases in which he observed the bulb everted and contained within the vagina. Both writers agree that the bulb has an inverted position in the queen, and Shafer notes that the reversed points of the dorsal plates of the bulb are pressed upward against the vaginal wall, thereby preventing an extraction of the bulb. Only the bulb is said to be found adhering to a mated queen, the other parts of the male organ, the cervix and the bursa, probably being torn away when the queen detaches herself from the drone. Both Shafer and Bishop believed that the spermatozoa are discharged into the vagina directly from the exposed end of the ejaculatory duct, but according to Zander the sperm are already stored in the bulb at the time of mating. The observations recorded above on the presence of a spermatic mass in the bulb of unmated drones suggests that the condition described by Zander is true probably in most cases. Differences in the age of drones examined may account for the observed presence or absence of spermatozoa in the bulb.

The mating of the honey bee is a subject that still needs investigation. We know nothing definitely concerning the function of the various structural parts of the complex endophallic organ of the drone, and, notwithstanding evidence that the bulb is everted into the vagina, it is difficult to imagine how such a thick-walled structure can be turned inside out.

The sting.-The sting of the bee has been so many times described that only a summarized account of its structure and mechanism need be given here; the writer has elsewhere (I933) given a full discussion of both phases of the subject. Though the hymenopterous sting is a modified ovipositor, its parts have long been known to students of the honey bee under special names, and these names will be used in the following description.

The act of stinging involves movements of the entire abdomen that accomplish the initial thrust of the shaft of the sting into the skin of the victim. The farther penetration of the shaft into the wound and the injection of the poison are produced by movements of the sting itself. The stinging organ (fig. 3I A) comprises two distinct parts: one is a large basal part by which the sting is attached within the sting chamber at the end of the abdomen, and which constitutes the motor apparatus; the other is the long, tapering shaft, which is the effective part of the organ, and is alone protracted from the abdomen during the act of stinging. The two parts of the sting are connected anteriorly by a pair of curved, flexible arms, and in the position of rest the shaft lies beneath the basal part and is mostly concealed within the latter. 

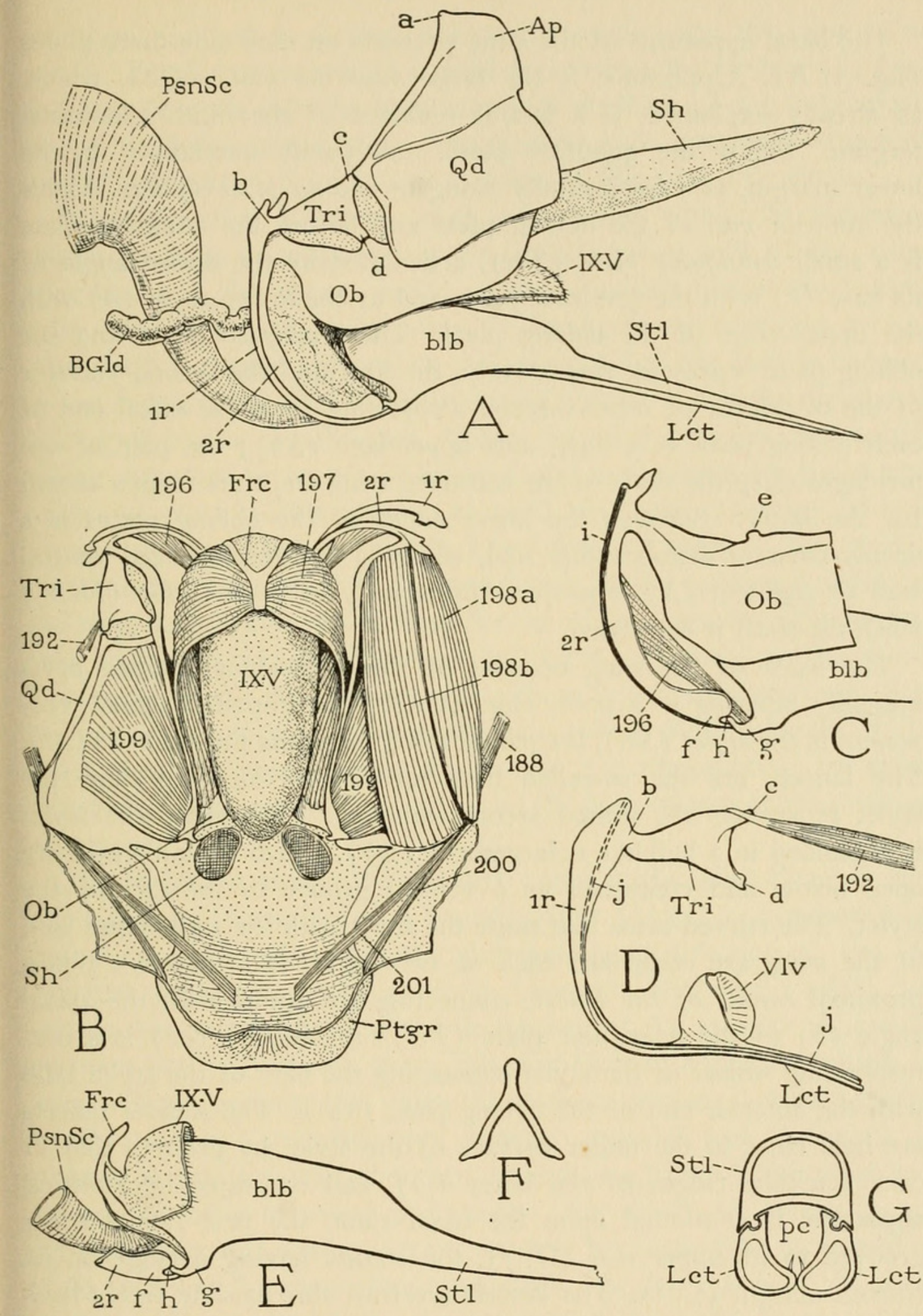

FIG. 31.-Structure of the sting. (From Snodgrass, 1933, with muscles renumbered.)

A, sting of worker, lateral view. B, sting and its muscles, with ventral wall of proctiger, dorsal view. $\mathrm{C}$, bases of oblong plate and bulb of stylet connected by second ramus (2r). $\mathrm{D}$, triangular plate and base of lancet connected by first ramus $(I r)$. $\mathrm{E}$, proximal bulbous part of stylet, showing basal articulation on second ramus, attachment of furcula, and entrance of duct of poison sack into base of bulb. F, furcula. G, cross section of distal part of shaft of sting.

$a$, angle of attachment of quadrate plate to spiracular plate of eighth segment; $A p$, apodeme of quadrate plate; $b$, apex of triangular plate; BGld, "alkaline" gland of sting; $b l b$, bulb of stylet; $c$, $d$, basal angles of triangular plate; $e$, fulcrum of triangular plate on oblong plate; $f$, articular lobe of second ramus; Frc, furcula; $g$, articular lobe of bulb; $h$, hinge between second ramus and bulb; $i$, ridge of second ramus; $j$, groove of first ramus; Lct, lancet; $O b$, oblong plate; $p c$, poison canal; $P s n S c$, poison sack; Ptgr, proctiger; $Q d$, quadrate plate; Ir, first ramus (basal arm of lancet); $2 r$, second ramus (basal arm of stylet); $S h$, sheath lobe; Stl, stylet; Tri, triangular plate. 
The basal apparatus of the sting presents on each side three plates (fig. 3I A). Uppermost is the large quadrate plate ( $Q d$ ), which, as already explained, is a lateral remnant of the ninth abdominal tergum. Below the quadrate plate, and partly overlapped by its lower margin, is a horizontally elongate oblong plate $(\mathrm{Ob})$. Above the anterior end of the oblong plate and before the quadrate plate is a small triangular plate (Tri), articulated by the dorsal angle of its base $(c)$ with the quadrate plate, and by the lower angle $(d)$ with the upper edge of the oblong plate. The triangular plate and the oblong plate represent, respectively, the first and the second valvifer of the ovipositor of other insects. Projecting from the distal end of each oblong plate is a long, soft appendage $(S h)$; the pair of appendages clasp the shaft of the retracted sting and thus form a sheath for the latter. Between the lower edges of the oblong plates is a deeply concave membranous fold, which is the unsclerotized ventral wall of segment $I X$; it projects posteriorly as a free lobe (IX.V) when the shaft is depressed.

The shaft of the sting consists of three closely appressed parts mutually tapering to a point (fig. 3I A). The unpaired upper component is the stylet $(S t l)$, the paired lower elements the lancets $(L c t)$. The lancets are the so-called first valvulae of an ovipositor, the stylet represents the united second valvulae. Proximally the stylet is expanded in a bulblike enlargement $(b l b)$, which contains a cavity open below and continued as a ventral groove to the end of the stylet. The curved arms that unite the shaft with the supporting base of the sting are composed each of two parts. The first (Ir) is a proximal ramus of the lancet, connecting the lancet with the apical angle $(b)$ of the triangular plate (Tri); the second ( $2 r$ ) is a corresponding ramus of the stylet connecting the base of the stylet bulb with the anterior end of the oblong plate $(O b)$. The slender lancets are held close to the under surface of the stylet by grooves that fit over tracklike ridges of the latter $(\mathrm{G})$, and the same interlocking apparatus is continued upon the basal rami, the first ramus being grooved to its upper end $(\mathrm{D}, j)$, the second having a ridge on its convex margin $(\mathrm{C}, i)$. The lancets are thus able to slide freely back and forth on the under side of the stylet. Between the stylet and the lancets is the poison canal of the sting $(G, p c)$, which expands proximally into the cavity of the bulb, and here receives the poison liquid from the great poison sack (A, PsnSc), or reservoir of the poison glands, which opens into the base of the bulb. Each lancet bears on its proximal part a valvelike lobe $(\mathrm{D}, V l v)$, the two lobes projecting side by side into the cavity of the bulb, where they serve 
to propel the poison through the poison canal when the sting is in action. Finally we must note a small forked rod, the furcula (E, Frc, $\mathrm{F}$ ), that projects into the body cavity at the base of the bulb above the neck of the poison sack. The furcula is an apodemal structure giving attachment to important muscles of the sting (B, Frc).

When the sting is not in use it is concealed within the abdomen, where its base is supported in the membranous wall of the sting chamber, and the upper angles of the quadrate plates are loosely articulated on the spiracular plates of the eighth segment (fig. $32 \mathrm{~A}$ ). The shaft is turned upward on the rami so that the bulb is encased in the membranous fold between the oblong plates, and the distal part is clasped by the sheath lobes. When the sting goes into action, the whole structure is swung downward and posteriorly on the dorsal angles of the quadrate plates $(a)$ until the basal apparatus takes a vetrical position with the sheath lobes directed upward (fig. $32 \mathrm{~B}$ ); simultaneously the shaft is depressed and, by the backward swing of the sting base, is protracted from the tip of the abdomen. The lancets now begin a rapid, alternate back-and-forth movement on the stylet. The action of the sting thus involves three separate movements: first the backward swing of the entire organ, second the depression of the shaft, third the movement of the lancets. On retraction of the sting, the shaft is replaced in the sheath, and the base returns to the position of repose.

The backward swing of the entire organ in the sting chamber was attributed by the writer in a former paper (1933) to pressure supposedly engendered within the abdomen by contraction of the abdominal segments. More recently, however, Rietschel (I937) has shown that specifically it is the depression of the seventh abdominal sternum that projects the sting. Manipulation of a dead specimen, in fact, will demonstrate that when the seventh sternum is turned downward, its broad anterior part, turning upward and backward, is pressed deeply into the abdomen against the base of the sting and forces the latter to swing posteriorly. The shaft, of course, is not protracted by this action, since it must at the same time be turned at right angles to the supporting apparatus. The depression of the shaft is effected by a pair of large, flat muscles arising on the inner surfaces of the oblong plates (fig. $3 \mathrm{I} \mathrm{B,} 197$ ) that curve anteriorly and mesally over the base of the bulb and are inserted on the furcula $(F r c)$. The tension of these muscles on the furcula pulls upward on the base of the bulb (fig. $32 \mathrm{C}$ ), and the shaft is turned downward on a pair of fulcral points $(h)$ at the lower angles of the bulb that abut against small lobes on the lower ends of the second rami $(2 r)$. The fulcral 
apparatus is shown in greater detail at $\mathrm{C}$ and $\mathrm{E}$ of figure $3 \mathrm{I}$, and the action of the depressor muscles (197) on the shaft is diagrammatically expressed at D and $\mathrm{E}$ of figure 32 . Mesad of the fulcrum each ramus of the stylet is continuous with the bulb by a slender, flexible connection. The opposite movement of the shaft, by which it is again turned upward and ensheathed between the oblong plates and their distal appendages, is effected by a pair of slender muscles stretched from the second rami to the fulcral lobes of the bulb (figs. 3I B, C, 32 C, D, I96), the insertion points of the muscles being sufficiently beyond the fulcral points to afford a short leverage on the shaft.

The movements of the lancets accomplish the penetration of the tip of the shaft into the victim, and the injection of the poison into the wound. They are produced by two pairs of large muscles in the basal apparatus that affect primarily the quadrate plates, and secondarily the triangular plates and the attached lancets. The muscles of one pair consist each of two large bundles of fibers (figs. 3I B, $32 \mathrm{C}, 198$ ) attached posteriorly, one laterally the other mesally, on the upper apodemal part of the quadrate plate, and anteriorly on the anterior end of the oblong plate. The muscles of the other pair (I99) are attached anteriorly by broad, spreading bases on the inner surfaces of the quadrate plates, and posteriorly on the posterior ends of the oblong plates. The alternate contraction of these two muscles on each side pulls the corresponding quadrate plate first forward and then backward, relative to the oblong plate; the movements of the quadrate plate are transmitted to the triangular plate (fig. 3I A, Tri) by the connection of the former with the upper basal angle of the latter $(c)$; the triangular plate, in turn, rotates on its ventral articulation $(d)$ with the oblong plate, and the final result is that the alternate up-anddown movement of the apical angle $(b)$ of the triangular plate is transmitted to the attached ramus of the lancet $(I r)$, which gives the lancet itself a back-and-forth movement on the stylet. The mechanism is illustrated diagrammatically at $\mathrm{F}$ and $\mathrm{G}$ of figure 32 . At $\mathrm{F}$ muscle I99, the retractor of the stylet, is contracted, the quadrate plate consequently pulled backward, the apex of the triangular plate lifted, and the lancet $(L c t)$ retracted by the pull on its basal ramus. At $G$ muscle 198 , the protractor of the lancet, is in contraction, the quadrate plate is pulled forward, the triangular plate depressed, and the lancet protracted. In a living bee, as may be seen in a freshly extracted sting, the movements of the quadrate plates appear as a rapid vibration, and the two lancets have alternately opposite motions. 
When a bee stings, the initial insertion of the tip of the out-thrust shaft into the skin of the victim is probably effected by a quick movement of the abdomen. The subsequent deeper penetration is the result of the successive alternating movements of the lancets, which
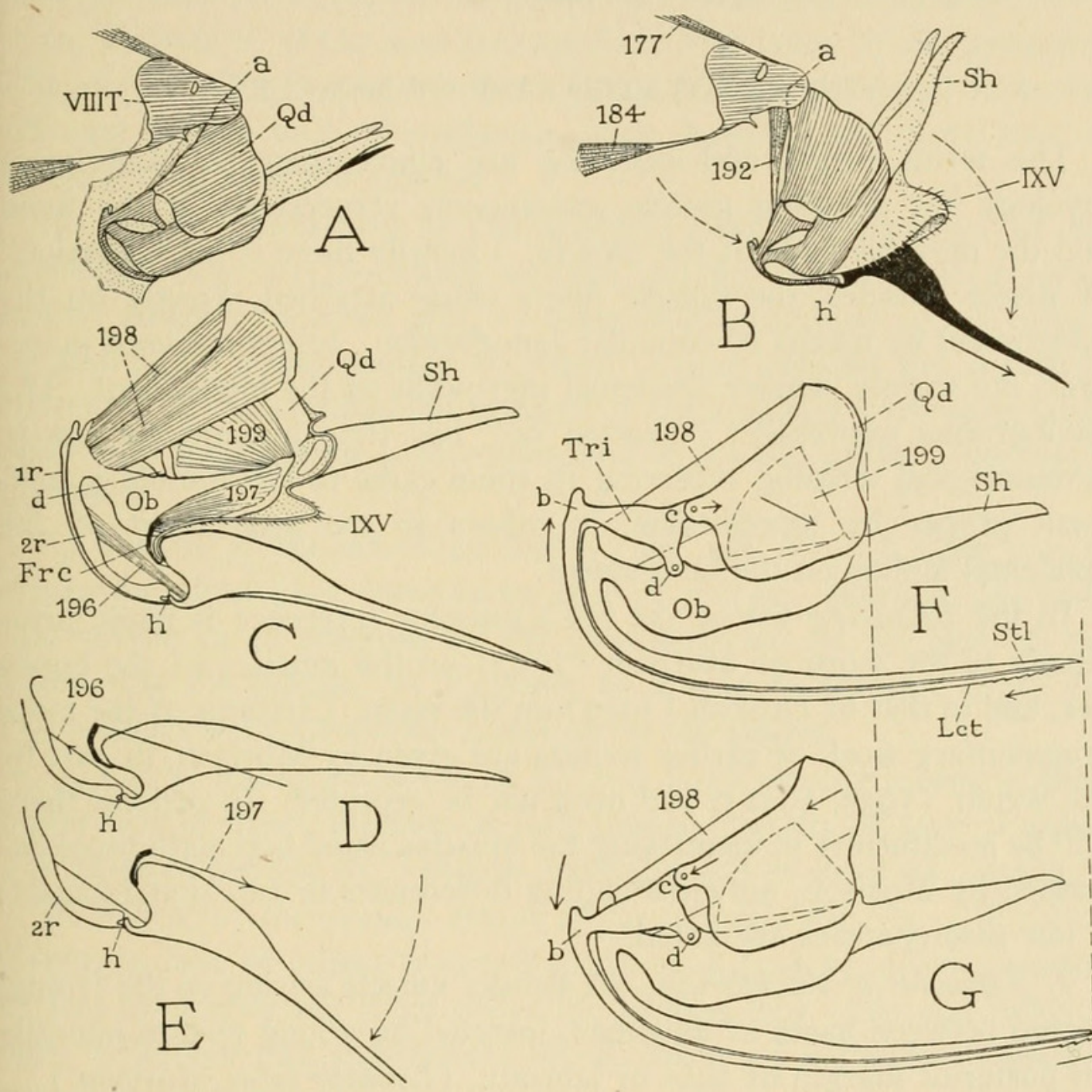

FIG. 32.-Mechanism of the sting.

A, sting in position of retraction within sting chamber of abdomen. B, sting protracted. C, right half of basal parts of sting, mesal view, shaft partly depressed, showing muscles of right side. D, shaft of sting, held in usual position by contraction of ramus muscle $(196)$. E, same in protracted position, shaft depressed on basal hinge with ramus $(h)$ by contraction of furcular muscle (197). F, diagram of sting and muscles of oblong plate: lancet retracted by contraction of muscle I99. G, same, lancet protracted by contraction of muscle 198 .

Lettering as on figure $3 \mathrm{I}$.

at each deeper insertion hold their gain by means of recurved barbs on their distal ends. The stylet, after the first jab, passively follows the lancets. The point of the shaft thus automatically goes deeper and deeper into the wound by the action of the lancets, and continues to 
do so even when the sting is detached from the body of the bee. At the same time the contents of the poison sack are poured into the bulb of the sting and driven through the poison canal by the action of the valves on the bases of the lancets. The poison escapes through a ventral cleft near the tips of the lancets.

\section{ANNOTATED LIST OF MUSCLES}

The terms origin and insertion are conventional in descriptive myology for denoting muscle attachments respectively at the fixed and the movable ends of the muscle. Literally there is no "insertion" of insect muscles, the muscle fibers being attached directly on the integument by means of cuticular tonofibrillae. Insect tendons, moreover, are merely slender apodemal ingrowths of the integument. The incongruous expression "inserted on" has thus become current in entomological writing, referring in some cases to the muscle attachment proper by tonofibrillae, in others to the attachment of the apodemal tendon on the integument.

In the following list of the bee's muscles reference is made principally to the work of Morison (I927) on the muscles of the honey bee, and to that of Duncan ( I939) on the wasp. Citations of the more fragmentary work of earlier writers are given by Morison, in part by the writer (1933, I94I), and need not be repeated. In general there will be no difficulty in identifying the muscles listed here with those described by Morison, notwithstanding differences in functional names; a few discrepancies are noted.

I. Adductor of the labrum.-A slender muscle arising on the frontal region between bases of antennae, inserted by a long tendon laterally on posterior margin of base of labrum. (Levator labri Morison.)

2, 3, 4, 5. Extrinsic muscles of the antenna (fig. 2 A, C).-Four muscles arising on anterior arm of tentorium, inserted on base of antenna.

6. Levator of the antennal flagellum (fig. $2 \mathrm{H}$ ).-A slender muscle arising on dorsal wall of scape, inserted dorsally on base of pedicel.

7. Depressor of the antennal Alagellum (fig. $2 \mathrm{H}$ ).-Origin on ventral wall of scape, insertion ventrally on base of pedicel.

8. Abductor of the mandible (fig. $3 \mathrm{I}$ ).-A flat muscle arising by spreading fibers on genal and postgenal walls of cranium behind lower part of compound eye, inserted by strong apodeme in membrane between mandibular base and pleurostomal margin of cranium (fig. $3 \mathrm{E}, 8 A p)$. 
9. Adductor of the mandible (fig. $3 \mathrm{H}$ ).-A larger muscle than the last, fibers arising in several groups behind upper part of compound eye, on postgena below level of foramen magnum, and one small group $(g a)$ on top of head, inserted by strong apodeme in articular membrane mesad of mandibular base (fig. I C, $q A p$ ).

IO. Promotor of the maxilla; cardinal protractor of the proboscis (fig. 4A).-Origin posteriorly on inner face of postgenal inflection forming lateral wall of proboscis fossa, inserted on short arm of cardo beyond articular process.

II I I2, I3. Adductors of the maxilla; stipital protractors of the proboscis (fig. $4 \mathrm{~A}$ ).-Three muscles arising on extreme anterior end of anterior tentorial arm, II inserted on proximal end of stipes, $I 2$ and $I 3$ on mesal margin of stipes. These muscles are primarily adductors of the maxilla, but because of the angulation between cardo and stipes they become protractors, and in the bee protract the entire proboscis.

14. Muscle of the maxillary palpus (fig. 4 B, F).-A large muscle arising in stipes $(\mathrm{F})$, inserted on base of palpus. Morison describes this muscle as an extensor of the galea inserted on the base of the latter near the palpus. In Vespula, according to Duncan, there are two muscles with a common insertion on the palpus.

15. Flexor of the galea (fig. $4 \mathrm{~F}$ ).-A large pinnate muscle arising posteriorly in stipes, inserted by long tendon laterally on base of galea. The galea of the bee, as in insects generally, has no extensor muscle.

I6. Flexor of the lacinia; tensor of the lacinial lobe (fig. 4 F, G).Origin in base of stipes, insertion on lever in base of lacinia; tension of this muscle on the lever raises the lacinial lobe $(l c l)$ and holds it taut. (Spanner des Segelhalters Wolff; flexor of the lacinia Duncan; not given by Morison.)

I7. Anterior adductor of the labium; retractor of the proboscis (fig. 7 A, D).-A long muscle arising on dorsal wall of cranium, tapering downward to strong tendon inserted on distal end of ligular bar of prementum $(h)$. It retracts the entire proboscis, also the ligula into the prementum, and recurves the glossal tongue. (Retractor linguae longus Wolff ; retractor ligulae superior Morison; flexor of the paraglossa Duncan.)

18. Posterior adductor of the labium; protractor of the labium (fig. 7 D).-Origin on extreme anterior end of anterior tentorial arm, inserted with muscle of opposite side by common tendon attached on base of prementum. Serves to adduct the labium, and probably also protracts it by flattening the angle between prementum 
and postmentum. (Protractor labii Morison; posterior labial adductor Duncan.)

19. Premental retractor of the ligula (fig. 7 A).-Arises laterally in base of prementum, tapers to tendinous insertion on ligular arm (h) of prementum just laterad of $I 7$. This muscle is probably the paraglossal muscle of the labium; functionally it is accessory to $I 7$ in retraction of the ligula: (Retractor linguae brevis Wolff; anterior flexor of the ligula Duncan; wrongly described by Morison as flexor palpi maxillaris.)

20. Flexor of the glossa, retractor of the tongue (fig. $7 \mathrm{~A}, \mathrm{~B}$ ).Origin posteriorly in base of prementum, insertion by long tendon close to side of base of glossal rod. The muscles of this pair serve to shorten the tongue or to roll it from side to side. (Smaller branch of retractor linguae biceps Wolff ; retractor ligulae inferior Morison; posterior flexor of the ligula Duncan.)

2I. Flexor of the labial palpus (fig. $8 \mathrm{~A}$ ).-A flat pinniform muscle arising on side of prementum, inserted by long tendon on rod in posterior wall of palpiger. It depresses the palpus and folds the latter back behind the stipes. (Extensor palpi maxillaris Morison; depressor of the labial palpus Duncan. Morison mistakenly assigns two muscles to the labial palpus in the bee. Duncan finds but one in Vespula.)

22. Muscle of the second segment of the labial palpus (fig. $8 \mathrm{~A}$ ).Origin in base of first palpus segment, insertion on base of second segment; flexes the second segment mesally, opposed by elasticity of the joint.

23. Dilator of the salivary syringe (fig. 7 A, C).-A flat muscle arising on anterior lateral margin of prementum, inserted medially on anterior wall of salivary syringe. (Protractor liguae Wolff; dilator ampullae superior Morison; dilator of the salivarium Duncan.)

24. Compressor of the salivary syringe (fig. $7 \mathrm{~A}, \mathrm{C}$ ).--Largest muscle in prementum, arising proximally, inserted distally on lateral margins of salivary syringe. Probably compresses the syringe by lateral tension. (Principal branch of retractor linguae biceps Wolff; dilator ampullae salivae posterior Morison; posterior muscle of salivarium Duncan.)

25. Retractor, or levator, of the epipharynx (fig. Io C).-A group of fibers arising on distal part of clypeus, diverging to insertions within the epipharynx. (Levator veli palatini Wolff.)

$26,27,28,29,30$. Dilators of the suctorium (fig. Io $\dot{\mathrm{C}}$ ).-Five large, paired bundles of short fibers arising on clypeus and inserted on anterior wall of cibarial region of sucking pump. 
3I. Compressors of the suctorium (fig. Io C).-Five thick bundles of fibers on anterior wall of cibarial region of sucking pump, the first transverse over the mouth, the others oblique between the dilators, attached laterally on oral plate $(o p l)$.

32. Protractor of the oral plate (fig. Io B, C).-Origin ventrally on a process of frontal aspect of base of anterior tentorial arm, insertion dorsally on upper part of pharyngeal arm $(y)$ of oral plate above frontal ganglion connective. (Lateral pharyngeal muscle Duncan.)

33. Retractor of the oral plate (fig. Io B, C).-A smaller muscle than the last arising medially on frons below median ocellus, inserted ventrally opposite 32 on arm of oral plate. (No corresponding muscle given by Duncan in the wasp.)

34, 35. Precerebral dilators of the pharynx (fig. Io C).-Two small muscles arising on frons, inserted on pharyngeal region of sucking pump just above frontal ganglion. (Frontal dilators of the anterior pharynx Duncan.)

36. Postcerebral muscle of the pharynx (fig. Io C).--Long slender muscle arising on vertex, inserted laterally on upper extremity of pharynx, probably mainly suspensory in function. (Dorsal dilator of the posterior pharynx Duncan.)

37. Posterior contractor of the pharynx (fig. Io C).-A long, median, fusiform muscle lying against posterior wall of pharynx, arising by slender tendon on median process of tentorial bridge, inserted ventrally on inner edge of oral plate. (Posterior dilator of the anterior pharynx Duncan.)

38. Parietal contractor of the pharynx (fig. Io C).-A broad sheet of fibers spreading over lateral and posterior walls of pharynx from two points of origin laterally on tentorial bridge, fibers going beneath circular muscles on lower half of pharynx to attachments on arms of oral plate. This muscle and the last are termed "dilators" of the pharynx in the wasp by Duncan, but their lengthwise distribution would suggest that they are contractors of the pharynx, though at the same time they may dilate the cibarium.

39. Constrictors of the pharynx (fig. Io C).-The layer of circular fibers investing the entire pharyngeal region of the sucking pump, continued on the oesophagus.

40. Superior phragmatic levator of the head (fig. I2 A).-One of a pair of submedian, parallel muscles arising on prephragma of mesonotum, inserted laterally on dorsal margin of foramen magnum. (Levator capitis horizontalis Morison.) 
4I. Inferior phragmatic levator of the head (fig. I2 A).-One of a pair of long muscles arising laterally on prephragma of mesonotum, converging beneath 40 to insertion in dorsal angle of foramen magnum. (Rotator capitis superior Morison.)

42. Pleural levator, or rotator, of the head (fig. I2 B, D).-A large, three-branched muscle arising on episternum, inserted by common tendon attached in laterodorsal notch of foramen magnum; none of its fibers observed to arise on endosternum as stated by Morison. (Rotator capitis inferior Morison; pleural elevator of the head Duncan.)

43. Endosternal levator, or rotator, of the head (fig. I2 B, C).A large muscle with wide base on dorsal surface of prothoracic endosternum, fibers convergent on long thick tendon attached in laterodorsal notch of foramen magnum above 42. (Levator capitis obliquus Morison; furcal elevator of the head Duncan.)

44. Depressor of the head (fig. I2 B).-A large muscle of two parts arising on dorsal surface of prothoracic endosternum anterior to 43 , inserted by strong tendon on ventral margin of foramen magnum. (Depressor capitis horizontalis Morison; first and second depressors of the head Duncan.)

45. Intersegmental dorsal muscle of the prothorax (figs. II F, I2 A).-A thick muscle arising on middle of pronotum, attached posteriorly on prephragma of mesonotum; probably depresses the pronotum. (Depressor prothoracis Morison; inner pronotal retractor of the mesonotum Duncan.)

46. Phragmatopleural muscle of the prothorax (fig. I2 A).Arises farthest laterad on prephragma of mesonotum, attached anteriorly on cervical apodeme of pleuron. (Lateral branch of levator prothoracis internus Morison; anterior notal elevator of the propleuron and head Duncan.)

47. A small prothoracic muscle attached close to 46 on cervical apodeme of pleuron (fig. I2 A, B) may be a branch of 48 , though its fibers appear to run along the under side of the latter.

48. Tergoepisternal muscle of the prothorax (fig. I2 A).-A large, straplike muscle arising mesally on posterior inflection of pronotum (not on phragma), attached anteriorly on horizontal apodeme of episternum (A, B). (Levator prothoracis externus Morison.)

49, 50. Protractors of the propectus (fig. I2 B, C).-Two fanshaped muscles arising anteriorly on side of pronotum, inserted on apex of pleural apophysis. (Depressor epistermi prothoracis anterior and posterior Morison; first and second posterior notal protractors of the propleuron Duncan.) 
5I. Adductor of the propleuron (fig. I2 B).- A large muscle arising mesally on straplike apodeme of prothoracic endosternum (fig. II E, $n)$, inserted anteriorly on cervical apodeme of pleuron. ( $R e$ tractor episterni prothoracis Morison; prothoracic furco-pleural muscle Duncan.) The muscles of this pair cannot be retractors of the episterna because of the endosternal attachments of the pleura.

52. Intersegmental ventral muscle of the prothorax; endosternal retractor of the propectus (fig. I2 C).-A slender muscle attached anteriorly on base of proendosternum, posteriorly on supraneural bridge of mesoendosternum (fig. 22 C). (Retractor prothoracis Morison; first furcal retractor of the propectus Duncan, the second retractor of Vespula absent in Apis.)

53. Lateral promotor of the fore coxa (fig. I3 C, D, G).-A broad muscle arising on episternum, inserted laterally on coxa anterior to pleural articulation.

54. Mesal promotor of the fore coxa (fig. I3 C, D).-A small muscle arising on side of endosternum, inserted on anterior mesal angle of coxal base.

55. First lateral remotor of the fore coxa (figs. I2 C, I3 C, D, G).Longest of the coxal muscles, arises laterally on pronotum at base of spiracular lobe (fig. I7 C), inserted on outer rim of coxa behind pleural articulation.

56. Second lateral remotor of the fore coxa (figs. II C, I2 C, I3 C, D, G).-A large muscle arising on under surface of supraneural bridge of endosternum (figs. I I C, I2 C), inserted on margin of coxal base close behind 55 (fig. I3 D, G).

57. Mesal remotor of the fore coxa (figs. I I C, I3 C, D).-Smallest of the coxal muscles, arising on upper end of pleural apophysis (fig. I I C), inserted mesally on posterior edge of coxal base (fig. I3 C, D).

58. Coxal retractor of the propectus (figs. II C, I2 C, I3 C, D, G). -A large muscle arising posteriorly on anterior part of median ridge of pterothoracic endosternum (fig. $22 \mathrm{C}$ ), the pair diverging forward to insertions on fore coxae mesad of pleural articulations (fig. I3 C, D). (Extensor coxae prothoracis Morison; mesosternal retractor of the propectus Duncan.)

59. Anterior levator of the fore trochanter (fig. $\mathrm{I} 3 \mathrm{E}, \mathrm{H}$ ).-Origin in coxa, inserted anteriorly on base of trochanter beyond coxal articulation.

60. Posterior levator of the fore trochanter (fig. I3 E, H).-Origin in coxa, inserted posteriorly on base of trochanter beyond coxal articulation. Morison mentions only one trochanteral levator, but two are present in both the drone and the worker. 
6I. Thoracic depressor of the fore trochanter (figs. II C, I2 C, I3 D, E, H). - A long muscle with origin on pleural apophysis of prothorax (not on endosternum as stated by Morison), going downward into coxa where fibers inserted on depressor apodeme of trochanter.

62. Coxal depressor of the fore trochanter (fig. I3 E, H).-A broad muscle occupying entire mesal wall of coxa, fibers inserted on depressor apodeme and apodemal plate of trochanter (fig. I3 E).

63. Reductor of the fore femur (fig. I3 H).-A short broad muscle arising on anterior wall of trochanter, inserted somewhat ventrally on posterior margin of base of femur.

64. Extensor of the fore tibia.-Origin dorsally in femur, inserted dorsally on base of tibia.

65. Flexor of the fore tibia.-Origin ventrally in femur, inserted on ventral genuflexor plate at base of tibia.

66. Productor of the fore tarsus (fig. $14 \mathrm{E}$ ).-A flat muscle arising on anterior wall of tibia, inserted anteriorly on base of tarsus.

67. Reductor of the fore tarsus (fig. I4 E).-A flat muscle arising on posterior wall of tibia, inserted posteriorly on base of tarsus.

68. Depressor of the fore tarsus (fig. I4 D, E).-A longer muscle than the last two, arising ventrally in tibia, inserted ventrally on base of tarsus.

69. Flexor of the pretarsal claws of the fore leg.-Fibers in two groups arising one in femur, the other in tibia, inserted on long slender tendon traversing tarsus, tibia, and femur from attachment at base of unguitractor plate of pretarsus.

70. External longitudinal dorsal muscle of the mesothorax (fig. I6 D).-A small submedian muscle traversing the mesocutellum.

7 I. Internal longitudinal dorsal muscle of the mesothorax; indirect depressor of the wing (fig. I6 A, C).-A huge muscle attached anteriorly on prephragma of mesonotum and on median area (fig. I6 B) of scutum, attached posteriorly on postphragma of mesonotum.

72. Tergosternal muscle of the mesothorax; indirect elevator of the wing (fig. I6 A, C).-A thick column of fibers attached dorsally on lateral area of mesoscutum (fig. I6 B), attached ventrally on sternal area of pleurosternal region.

73. Occlusor of the first spiracle (fig. I7 C, F).-A slender muscle arising on anterior margin of mesepisternum (C), inserted in spiracular membrane in front of spiracular aperture $(\mathrm{F})$.

74. Pleuroalar muscle of the mesothorax (fig. I9 C).-A small, two-branched muscle arising on anterior margin of mesepisternum 
(fig. I7 C), inserted on ventral side of humeral complex of first wing (fig. 19 C).

75. Pleurotergal muscle of the mesothorax (fig. I7 E).-A large, fan-shaped muscle arising on episternum, the fibers convergent to short tendon attached on lateral margin of posterolateral plate of scutum (fig. $19 \mathrm{~F}$ ). (Retractor scutelli mesothoracis Morison.)

76. Pleuroaxillary muscles of the mesothorax; flexors of the fore wing (figs. I7 E, I9 $\mathrm{H}$ ). - Three small muscles arising on mesopleuron, inserted by separate tendons on third axillary of wing base.

77. Basalar muscle of the mesothorax (figs. I7 E, I9 G).-A broad muscle with spreading fibers arising ventrally on anterior part of episternum, inserted dorsally by strong tendon on basalar sclerite.

78. Muscle of the axillary lever of the mesothorax (fig. $20 \mathrm{~A}, \mathrm{~B}$ ).Origin by broad base ventrally on mesothoracic arm of pterothoracic endosternum, insertion dorsally on end of lever sclerite of fourth axillary. (Musculus furco-lateralis mesothoracis Morison.) In nonapoid Hymenoptera this muscle is a muscle of the postphragma (fig. $20 \mathrm{C}$ to $\mathrm{H})$.

79. Pleurosternal muscle of the mesothorax (fig. I7 G).-A mass of short fibers arising on posterior epimeral area and on pleural apophysis of mesothorax, attached on end of mesothoracic arm of pterothoracic endosternum.

80. Lateral promotor of the middle coxa (figs. I7 G, 22 C).-A slender muscle arising on the small pleural apophysis of mesopleuron, inserted laterally on coxal base anterior to pleural articulation.

8I. Mesal promotor of the middle coxa (fig. $22 \mathrm{C}$ ). - Smallest of the mesocoxal muscles, arising on basal plate of endosternum, inserted mesally on anterior margin of coxa.

82. Lateral remotor of the middle coxa; coxosubalar muscle of the mesothorax (figs. I7 G, $22 \mathrm{C}$ ). - A long muscle attached ventrally on base of coxa just posterior to pleural articulation, attached dorsally by long tendon on subalar sclerite. Probably has little motor effect on the coxa; deflects the wing posteriorly by indirect tension on the third axillary. (Flexor coxae mesothoracis Morison; no corresponding muscle given by Duncan in mesothorax of the wasp.)

83. Mesal remotor of the middle coxa (fig. $22 \mathrm{C}$ ). - - Largest of the coxal muscles, origin on basal plate of endosternum, insertion mesally on posterior margin of base of coxa.

84. Anterior levator of the middle trochanter. (fig. $22 \mathrm{D}$ ).-Origin in lower part of coxa, insertion anteriorly on base of trochanter beyond coxal articulation. 
85. Posterior levator of the middle trochanter (fig. 22 D).-Origin posteriorly in coxa, insertion posteriorly on base of trochanter beyond coxal articulation.

86. Thoracic depressor of the middle trochanter (fig. $22 \mathrm{C}, \mathrm{G}$ ).Origin dorsally on side of endosternum (C), insertion on end of depressor apodeme of trochanter $(\mathrm{G})$.

87. Anterior coxal depressor of the middle trochanter (fig. $22 \mathrm{D}$, G).-Origin anteriorly in base of coxa (D), insertion on base of depressor apodeme of trochanter $(\mathrm{G})$.

88. Posterior coxal depressor of the middle trochanter (fig. $22 \mathrm{D}$, G).-Origin posteriorly in base of coxa (D), insertion on base of depressor apodeme of trochanter $(G)$.

89. Reductor of the middle femur (fig. 22 D, G).-Origin anterodorsally in trochanter, insertion posteroventrally on base of femur.

90. Extensor of the middle tibia (fig. $22 \mathrm{~L}$ ). - A long pinnate muscle arising on dorsal wall of femur, inserted by axial tendon on dorsal process of base of tibia.

91. Flexor of the middle tibia (fig. $22 \mathrm{~J}$ ).-A muscle similar to but larger than the extensor, arising on ventral wall of femur, inserted by its tendon on ventral genuflexor plate $(g f)$ at base of tibia.

92. Productor of the middle tarsus (fig. $22 \mathrm{E}$ ). - A slender pinnate muscle arising anteriorly in tibia, inserted by tendon anteriorly on base of tarsus.

93. Reductor of the middle tarsus (fig. $22 \mathrm{E}$ ).- Similar to the productor, arising posteriorly in tibia, inserted posteriorly on base of tarsus.

94. Depressor of the middle tarsus (fig. $22 \mathrm{~F}$ ).-A broad pinnate muscle arising on ventral wall of tibia, inserted ventrally on base of tarsus.

95. Flexor of the pretarsal clazes of the middle leg.-Same as in fore leg and hind leg, one group of fibers arising in base of femur, another in tibia, inserted by long tendon on unguitractor plate at base of pretarsus.

96. Retractor of the mesothoracic postphragma (figs. I6 C, $27 \mathrm{C}$ ).A small fan-shaped muscle arising by narrow end on posterior median process of propodeum, fibers spreading anteriorly and laterally to attachments on side of postphragma of mesothorax. (Musculus retractor mesophragmati Morison, mesophragma retractor muscle Betts.) The muscles of this pair probably are the longitudinal dorsal muscles of the metathorax with their propodeal attachments shifted posteriorly. 
97. First tergosternal muscle of the metathorax (fig. 2I F, G).Origin by broad base on metathoracic arm of endosternum, insertion on anterior angle of lateral plate of metanotum (E).

98. Second tergosternal muscle of the metathorax (fig. 2I F, G).Origin on metathoracic endosternal arm posterior to 97 , insertion on posterior angle of lateral notal plate (E).

99. Third tergosternal muscle of the metathorax (fig. 2I F).-Attached ventrally by narrowed end on small tongue $(c)$ of metathoracic arm of endosternum, fibers spreading dorsally to triangular lateral area of metanotum.

100. Pleuroaxillary muscle of the metathorax; flexor of the hind wing (fig. 21 B, H).-A small, three-part, fan-shaped muscle arising on anterior marginal inflection of metapleuron, fibers converging to small nodule in membrane of wing base $(a)$ close to third axillary.

IOI. Basalar muscle of the metathorax (fig. 2I H).-A large muscle arising by broad base on apodemal lobe of metathorax behind mesocoxal cavity (fig. I6 $\mathrm{H}, r$ ), inserted dorsally by a strong tendon on basalar sclerite (fig. 2I B).

I02. Pleurosubalar muscle of the metathorax (fig. 2I C, H).-A broad, thick, fan-shaped muscle arising on metapleuron, fibers convergent to narrow insertion on subalar sclerite. (Not given by Morison; two corresponding muscles in Vespula, Duncan.)

103. Lateral promotor of the hind coxa (fig. $24 \mathrm{~A}, \mathrm{~B}$ ).- A fanshaped muscle arising on ridge between mesopleuron and propodeum, the convergent fibers inserted laterally on anterior margin of coxa.

104. Mesal promotor of the hind coxa (fig. $24 \mathrm{~A}, \mathrm{~B}$ ).-Origin on metasternal component of pterothoracic endosternum, insertion mesally on anterior coxal margin.

105. Lateral remotor of the hind coxa; coxosubalar muscle of the metathorax (fig. 24 A, B).-A long muscle attached below on coxa immediately behind pleural articulation, inserted dorsally by long tendon on apodeme of subalar sclerite (fig. 2I B, C). Deflects the wing posteriorly. (Wrongly said by Morison to arise on endosternum.)

I06. Mesal remotor of the hind coxa (fig. $24 \mathrm{~A}, \mathrm{~B}$ ).-Origin by wide base on metasternal component of pterothoracic endosternum, inserted by broad tendon in articular membrane close to posterior mesal angle of base of coxa.

107. Anterior levator of the hind trochanter (fig. 24 C, D).-Origin anteriorly in coxa, insertion by tendon in dorsal membrane of coxotrochanteral joint beyond anterior coxal articulation (D). 
108. Posterior levator of the hind trochanter (fig. 24 C, D).Origin posteriorly in coxa, insertion in dorsal membrane of coxotrochanteral joint beyond posterior coxal articulation (D).

I09. Thoracic depressor of the hind trochanter (fig. $24 \mathrm{~A}, \mathrm{C}$ ).Origin on metathoracic arm of pterothoracic endosternum (A), fibers convergent into coxa to insertion on long depressor apodeme of trochanter (D, $A p)$.

IIO. Coxal depressor of the hind trochanter (fig. $24 \mathrm{C}, \mathrm{E}) .-\mathrm{A}$ broad muscle arising ventrally in coxa, the convergent fibers inserted on base of depressor apodeme of trochanter (E).

III. Reductor of the hind femur (fig. $24 \mathrm{C}$ ). - A short thick muscle arising anterodorsally in trochanter, inserted ventroposteriorly on apodemal inflection of base of trochanter $(\mathrm{F}, A p)$.

II2. Extensor of the hind tibia (fig. $24 \mathrm{H}$ ). - A long pinnate muscle arising along dorsal wall of femur, inserted by tendon on dorsal knob of base of tibia.

II3. Flexor of the hind tibia (fig. $24 \mathrm{H}$ ).-A large pinnate muscle lying in ventral half of tibia, fibers attached anteriorly and posteriorly, inserted by strong axial tendon on ventral genuflexor plate at base of tibia ( $\mathrm{G}, \mathrm{H}, g f$ ).

II4. Promotor of the hind tarsus (fig. $24 \mathrm{~J}, \mathrm{~K}, \mathrm{~L}$ ).- - Origin ventrally on anterior wall of wide distal part of tibia $(\mathrm{K}, \mathrm{L})$, inserted anteriorly on base of tarsus $(\mathrm{J})$.

II5. Levator of the hind tarsus (fig. $24 \mathrm{~J}, \mathrm{~K}, \mathrm{~L}$ ).-A fan-shaped muscle arising on posterior wall of distal part of tibia $(K)$, inserted posteriorly by narrow stalk on base of tarsus but above level of tibial articulation $(J)$. Corresponds with the reductor of the other legs.

II6. Depressor of the hind tarsus (fig. $24 \mathrm{~J}, \mathrm{~K}, \mathrm{~L}$ ).-Much larger than the other two tarsal muscles, arises on anterior wall of tibia $(\mathrm{K})$, inserted ventrally on base of tarsus $(\mathrm{J}, \mathrm{L})$.

II7. Flexor of the pretarsal claws of the hind leg (fig. $24 \mathrm{H}, \mathrm{K}$ ).As in the other legs, a two-branched muscle, one branch arising posteriorly in base of femur $(\mathrm{H}, I I 7 a)$, the other posteriorly in base of tibia $(\mathrm{K}, I I 7 b)$, both branches inserted on long apodemal tendon extending through tarsus and tibia into femur from its origin in articular membrane at base of unguitractor plate of pretarsus, as in middle leg (fig. $25 \mathrm{~B}$, 95 $\mathrm{Ap}$ ).

II8. Median intersegmental ventral muscle of the metathorax; median depressor of the abdomen (fig. $27 \mathrm{E}$ ).-A long muscle arising anteriorly on metasternal component of pterothoracic endosternum, inserted posteriorly by broad tendon medially on sternum of petiole segment (IIS). 
II9. Lateral intersegmental ventral muscle of the metathorax; ventral lateromotor of the abdomen (fig. $27 \mathrm{E}$ ).-A smaller muscle arising on median ridge of metasternum (in drone), inserted by long tendon laterally on sternum of petiole segment (IIS).

I20. Outer dorsal muscle of the propodeum; median levator of the abdomen (fig. 27 B).-A large muscle with spreading fibers arising on lateral wall of propodeum, inserted by strong tendon on median ridge of pocket on dorsal side of petiole (D, F, G). (Petiole muscle Betts; imusculus propodei retractor dorsalis postabdominis Morison.)

I2I. Inner dorsal muscle of the propodeum; dorsal lateromotor of the abdomen (fig. $27 \mathrm{~B}$ ).-A two-branched muscle arising laterally on propodeum at base of $I 20$, inserted by long thick tendon on lateral angle of tergal plate of petiole $(\mathrm{G})$.

I22. Occlusor of the propodeal spiracle (fig. $27 \mathrm{~B}$ ). - A short intraspiracular muscle.

I23. Dilator of the propodeal spiracle (fig. 27 B).-A long slender muscle arising from small process on dorsal margin of hind coxal cavity.

I24. Median internal dorsal muscle of abdominal segment II (fig. 28 A).

I25. Lateral internal dorsal muscle of abdominal segment II (fig. $28 \mathrm{~A})$.

I26. External dorsal muscle of abdominal segment II (fig. $28 \mathrm{~A}$ ).Runs transversely in fold between tergum $I I$ and tergum $I I I$.

I27. Occlusor of the spiracle of abdominal segment II.-A short intraspiracular muscle.

I28. Dilator of the spiracle of abdominal segment II (fig. $28 \mathrm{~A}$ ).A long slender muscle arising ventrally on lateral margin of sternum II.

I29. Lateral muscle of abdominal segment II (fig. $28 \mathrm{~A}$ ). - The only tergosternal muscle of segment II, being attached on dorsal surface of sternum it is probably the third lateral of other segments.

I30. Median internal ventral muscle of abdominal segment II (fig. 28 A).-The only internal ventral muscle in segment $I I$ of the female.

I3I. Lateral internal ventral muscle of abdominal segment II (fig. 29 B).-Present in the male only.

I32. External ventral muscle of abdominal segment II (fig. $28 \mathrm{~A}$ ). -Runs transversely from posterior part of sternum $I I$ to anterior apodeme of sternum $I I I$.

The muscles of the next four abdominal segments are repeated alike in each of these segments, and are present in both the male and the female; they are numbered in the female (fig. $28 \mathrm{~A}$ ), and may 
easily be identified by comparison in the male (fig. 29 B), though the muscles of the male are much larger and the second and third laterals are not crossed.

I33, I44, I55, I66. Median internal dorsal muscles of abdominal segments III to $V I$ (fig. $28 \mathrm{~A}$ ).

I34, I45, I56, I67. Lateral internal dorsal muscles of abdominal segments III to $V I$ (fig. $28 \mathrm{~A}$ ).

I35, I46, I57, I68. External dorsal muscles of abdominal segments III to VI (fig. $28 \mathrm{~A}$ ).-Oblique in anterior segments, reversed in posterior segments; origins posteriorly on anterior tergum, insertion anterior on anterior apodeme of posterior tergum.

$136,147,158,169$. Occlusors of the spiracles of abdominal segments III to VI (not shown in figures).

I37, I48, I59, I70. Dilators of the spiracles of abdominal segments III to VI (fig. $28 \mathrm{~A}$ ).- - Origins on long lateral apodemes of the sterna.

$I 3^{8}, I 49, I 60, I 7 I$. First lateral mscles of abdominal segments III to $V I$ (figs. $26 \mathrm{D}, 28 \mathrm{~A}$ ).--Reversed tergosternal muscles arising ventrally on lower parts of terga, inserted dorsally on lateral apodemes of corresponding sterna.

I39, I50, I6I, I72. Second lateral muscles of abdominal segments III to VI (fig. $28 \mathrm{~A}$ ).-External tergosternal muscles attached ventrally on lateral margins of the sterna; dorsoventral in the male (figs. $26 \mathrm{E}, 29 \mathrm{~B}$ ), oblique in the female (fig. $28 \mathrm{~A}$ ).

I40, I5I, I62, I73. Third lateral muscles of abdominal segments III to $V I$ (fig. $28 \mathrm{~A}$ ).--Internal tergosternal muscles attached ventrally in posterolateral angles of dorsal surfaces of sternal plates (fig. $26 \mathrm{E}$ ), parallel with second laterals in the male (figs. $26 \mathrm{E}, 29 \mathrm{~B}$ ), crossing the latter obliquely in the female (fig. $28 \mathrm{~A}$ ).

$I 4 I, I 52, I 63, I 74$. Median internal ventral muscles of abdominal segments III to $V I$ (fig. $28 \mathrm{~A}, \mathrm{~B}$ ).- In each segment the median ventrals form a $\mathbf{V}$ with the convergent ends posterior (B); in the male these muscles are wide bands of fibers.

I42, I53, I64, I75. Lateral internal ventral muscles of abdominal segments III to $V I$ (fig. $28 \mathrm{~A}, \mathrm{~B}$ ). - The lateral ventrals are oblique in an opposite direction from the median ventrals, the two sets forming in each segment an $\mathbf{M}$.

I43, I54, I65, I76. External ventral muscles of abdominal segments III to VI (fig. $28 \mathrm{~A}$ ). - Short reversed muscles from posterior parts of sterna to under surfaces of anterior apodemes of preceding sterna.

The musculature of abdominal segments $V I I$ to $I X$ is unlike in the two sexes, in some cases also between queen and worker, and differs from that of the preceding segments by the absence of certain muscles 
and the presence of special muscles in connection with the ovipositor and the male genital organ.

I77. Lateral internal dorsal muscle of abdominal segment VII.In the drone (fig. $29 \mathrm{~B}$ ) as in preceding segments; in the queen (fig. $28 \mathrm{E}$ ) attached on anterior part of spiracular plate of segment VIII; absent in the worker (C).

I78. Median internal dorsal muscle of abdominal segment VII.In the drone (fig. $29 \mathrm{~B}$ ) as in preceding segments; in the queen (fig. $28 \mathrm{E}$ ) a small muscle attached on spiracular plate of segment VIII near spiracle; in the worker (fig. $28 \mathrm{C}$ ) a much larger muscle similarly attached.

I79. External dorsal muscle of abdominal segment VII.-As in preceding segments in the drone; in the female a reversed dorsoventral muscle from lower edge of tergum VII to upper angle of spiracular plate of segment $V I I I$ (fig. $28 \mathrm{C}, \mathrm{E}$ ).

I80, I8I. Spiracular muscles of abdominal segment VII (fig. $28 \mathrm{C}$ ). -As in preceding segments.

I82. First and second lateral muscles of abdominal segment VII.First lateral present as a reversed muscle inserted dorsally on lateral apodeme of sternum $V I I$ in both the drone and the worker, but very small in the latter (No. $5 b$ of Rietschel, I937). The muscle designated 182 in the worker (fig. $28 \mathrm{C}$ ) also is attached on the lateral apodeme of sternum $V I I$, but arises posteriorly on the tergum and is probably the second lateral of segment $V I I$.

I83. Third lateral muscle of abdominal segment VII (fig. $28 \mathrm{C}$ ).Present in both the male and the female as a dorsoventral muscle between tergum and sternum.

I84. Intersegmental sternotergal muscle of abdominal segment VII (fig. $28 \mathrm{~B}, \mathrm{C}$ ).-Present in the female only; origin anteriorly on sternum $V I I$ in line with lateral ventral muscles of preceding segments (B), inserted posteriorly on anterior ventral angle of spiracular plate of segment VIII.

185. Median internal ventral muscle of abdominal segment VII (fig. $28 \mathrm{D}$ ).-Present in the queen only, attached posteriorly on lateral genital pouch.

186. Lateral internal ventral muscle of abdominal segment VII (fig. 29 B, D).--Present in the male only; a short muscle arising on sternum $V I I$ at base of anterior apodeme (B), inserted posteriorly on long lateral arm of sternum VIII.

I87. Median internal dorsal muscle of abdominal segment VIII.A short muscle in the male (fig. 29 B) attached posteriorly on apodeme of small tergite of segment $I X$; represented in the worker (fig. $28 \mathrm{C}$ ) 
and the queen (E) by a muscle $(I 87)$ from spiracular plate of segment $V I I I$ to quadrate tergal plate of segment $I X$.

188. Lateral internal dorsal muscle of abdominal segment VIII.Absent in the male, perhaps represented in the worker (fig. $28 \mathrm{C}$ ) and the queen (E) by a muscle (I88) from upper angle of spiracular plate to posterior dorsal angle of quadrate plate of segment $I X$ (fig. 3I B).

I89. Occlusor of the spiracle of abdominal segment VIII (fig. $28 \mathrm{C}, \mathrm{E})$. - A short intraspiracular muscle.

190. Dilator of the spiracle of abdominal segment VIII (fig. $28 \mathrm{C}$, E).- In the worker and the queen the dilator of the spiracle of segment VIII arises in the ventral anterior angle of the tergal spiracular plate, not on the sternum as in preceding segments.

I9I. Lateral muscle of abdominal segment VIII (fig. 29 B, D).Present in the male only, apparently represents the second tergosternal muscle of preceding segments.

192. Tergovalvifer muscle of abdominal segment VIII.-Present in the female only, arising on posterior ventral angle of spiracular plate (fig. $28 \mathrm{C}, \mathrm{E}$ ), goes anteroventrally to insertion on dorsal margin of triangular plate (first valvifer) of sting (fig. 3I D).

193, 194. Tergovaginal muscles of abdominal segment VIII.-Two slender muscles present in the queen only, arising on anteroventral part of spiracular plate (fig. $28 \mathrm{E}$ ), inserted on dorsal wall of vagina (D).

195. Lateral internal ventral muscle of abdominal segment VIII (fig. 29 B, D).-A short muscle present in the male only, arising laterally on sternum $V I I I$, inserted on lateral angle of parameral plate $(l p)$.

196. Levator of the shaft of the sting (fig. 3I B, C).-A small muscle arising on middle of ramus of stylet, inserted on small process $(\mathrm{C}, g)$ of base of stylet bulb a short distance beyond hinge $(h)$ between ramus and bulb.

197. Depressor of the shaft of the sting (fig. 3I B).-A large muscle arising posteriorly on oblong plate of sting, curving anteriorly and dorsally over base of stylet bulb to insertion on furcula (Frc). A small branch of this muscle is described by Rietschel (1937, No. $I g b)$ as arising laterally on the oblong plate.

198. Protractor of the lancet of the sting (figs. $3 \mathrm{I} \mathrm{B}, 32 \mathrm{C}$ ). $-\mathrm{A}$ large muscle of two parts attached posteriorly on outer and inner surfaces of upper, apodemal part of quadrate plate; attached anteriorly on anterior end of oblong plate at base of second ramus (2r). Pulls the quadrate plate forward, depresses the triangular plate, and pro- 
tracts the lancet (fig. $3^{2} \mathrm{G}$ ). The two parts of this muscle are anatomically two muscles, but functionally a single muscle. The upper part has mistakenly been said (Betts, Morison, and others) to be attached on the triangular plate.

199. Retractor of the lancet of the sting (figs. 3I B, $32 \mathrm{C}$ ).-A broad fan-shaped muscle arising anteriorly on quadrate plate, inserted posteriorly on upper edge of oblong plate. Pulls the quadrate plate backward, elevates the triangular plate, and retracts the lancet (fig. $32 \mathrm{~F}$ ).

200, 20I. Muscles of the proctiger in the female (fig. 3I B).-Two long slender muscles arising on posterior dorsal angle of the quadrate plate (tergite of segment $I X)$, inserted laterally and ventrally on lower wall of proctiger.

202. Median dorsal muscle of abdominal segment IX in the male (fig. 29 B).-A short muscle from apodeme of small tergite of segment $I X$ to side of proctiger. This muscle falls in line with the median dorsals of the preceding segments, and probably is represented by muscles 200 and 201 in the female.

203, 204. Phallic muscles of the male (fig. 29 D).-Two small muscles, or divergent parts of one muscle, arising ventrally on parameral plate $(l p)$, inserted dorsally on end of apodeme of penis valve (pv).

205. Muscle of the mucus gland of the male (fig. 29 D).-A slender muscle from apodeme of penis valve to mucus gland just laterad of end of vas deferens.

\section{ABBREVIATIONS USED ON THE FIGURES}

$A$, anal vein.

Ac, antecosta.

af, antennafer.

$A n$, anus.

$A N P$, anterior notal wing process.

Ant, antenna.

$A p$ or $a p$, apodeme.

$a p a$, apodeme of aedeagus.

$A r$, arolium.

arc, arcus

$a t$, anterior tentorial pit.

$A T$, anterior tentorial arm.

$a u$, auricle.

aux, auxilia.

$A x$, axillary sclerite of wing base ( $A A x, 2 A x, 3 A x, 4 A x$, first, second, third, and fourth axillaries).

$A x C$, axillary cord.

$a x l v$, axillary lever. 
$B a$, basalare.

$b c$, bursal cornua, pneumapophysis.

BGld, "alkaline" gland of sting.

$b i b$, biblike suboral flap.

$b l b$, bulb of sting.

$B l b$, bulb of endophallus.

$\mathrm{Br}$, brain.

Brs, bursa of endophallus.

$B s$, basisternum.

Btar, basitarsus.

$C$, costa, marginal vein of wing.

$\mathrm{Ca}$, corpus allatum.

$\mathrm{Cb}$, cibarium (suctorium of cibario-pharyngeal pump).

$c b l$, pollen basket (corbicula) of hind tibia.

$C c$, corpus cardiacum.

Cer, neck of endophallus.

$C d$, cardo.

$C l p$, clypeus.

$c n j$, conjunctiva.

$C v x$, neck, cervix.

$C x$, coxa.

$C x C$, coxal cavity (where coxa removed from body).

$C x P$, pleural coxal process.

$D e j$, ductus ejaculatorius.

$d l c b$, dilator muscles of cibarium.

dlphy, dilator muscles of pharynx.

$D T$, dorsal arm of tentorium.

$E$, compound eye.

Endst, endosternum.

Enph, endophallus.

Ephy, epipharynx.

Epm, epimeron (epm, subdivision of epimeron).

Eps, episternum.

$E R$, epistomal ridge.

es, sulcus of epistomal ridge (epistomal "suture").

$\mathrm{Fbl}$, flabellum.

$f c$, food canal of proboscis.

$F C$, food channel on base of proboscis.

$\mathrm{Fl}$, flagellum.

$f l b$, fimbriate lobe of endophallus.

$F m$, femur.

For, foramen magnum.

$F r$, frons.

Frc, furcula.

FrGng, frontal ganglion.

$F s$, furcasternum. 
$G a$, galea.

$\mathrm{Ge}$, gena.

$g f$, genuflexor plate.

Gls, labial tongue (glossae).

$H$, head.

$H B$, hypostomal bridge.

Hphy, hypopharynx.

$I b S$, infrabuccal sac.

$I S$, sternal plate of propodeum.

$I T$, tergal plate of propodeum.

$I X . V$, venter of ninth abdominal segment.

$L$, leg.

$L b$, labium.

LbPlp, labial palpus.

$L c$, lacinia.

$l c l$, lacinial lobe.

$L c t$, lancet (first valvula).

Lm, labrum.

$l O$, lateral ocellus.

$l p$, lamina parameralis.

$L r$, lorum.

$L s p$, lamina spiracularis (tergite of eighth abdominal segment).

$l v r$, lever of lacinia.

$M b$, interseg'mental membrane.

$M C u$, mediocubital vein.

$M d$, mandible.

$M d C$, cavity where mandible removed from head.

MdGld, mandibular gland.

$m n$, manubrium of arolium.

$m O$, median ocellus.

Mth, mouth.

$M x$, maxilla.

$M x P l p$, maxillary palpus.

$N$, notum (thoracic tergum).

$n f$, neural foramen.

$N R$, notal ridge.

$n s$, sulcus of notal ridge (scutoscutellar "suture").

$N v$, nerve.

$O$, ocellus.

$O b$, oblong plate of sting (second valvifer).

Oc, occiput.

$o c c$, occipital condyle.

Odc, oviductus communis.

$O d l$, oviductus lateralis. 
$O e$, oesophagus.

$O p$, operculum of first spiracle.

$o p l$, oral plate.

$p c$, poison canal of sting.

$P d c$, pedicel of antennal flagellum.

$P F$, proboscis fossa.

Pge, postgena.

$P g l$, paraglossa.

$P g L$, postgenal lobe.

$P h$, phragma ( $I P h$, first phragma, $2 P h$, second phragma).

Phtr, phallotreme.

Phy, pharynx.

$P l$, pleuron ( $p l$, subdivision of pleuron).

pla, pit of pleural apophysis.

$P l A$, pleural apophysis.

Plf, palpifer.

Plg, palpiger.

pln, planta.

$P l p$, palpus.

$P l R$, pleural ridge.

$p l s$, sulcus of pleural ridge (pleural "suture").

Pmr, paramere.

Pmt, postmentum.

$P N$, postnotum.

Poc, postocciput.

pos, postoccipital suture.

$p r$, pollen press.

Prb, proboscis.

Prmt, prementum.

PsnSc, poison sack.

$p t$, posterior tentorial pit.

Ptar, pretarsus.

Ptgr, proctiger.

$p v$, penis valve (lamina aedeagalis).

$Q d$, quadrate plate of sting (tergite of ninth abdominal segment).

$r$, ramus of sting ( $I r$, ramus of lancet; $2 r$, ramus of stylet).

$R$, radius.

ras, rastellum.

$r d$, glossal rod.

$R d p$, reduplication of posterior margin of notum.

$R N v$, recurrent nerve.

$S$, sternum.

$S a$, subalare.

$S A$, sternal apophysis.

$s c$, salivary canal of tongue.

$\mathrm{Scl}$, scutellum. 
Scp, scape.

$S c t$, scutum ( $s c t$, subdivision of scutum).

$s f$, scutal fissure (trans-scutal "suture").

Sga, subgalea.

$S h$, sheath lobe of sting.

$S I D$, salivary duct.

$\mathrm{SlO}$, salivary orifice.

$S l v$, salivarium (salivary syringe).

$S p$, spiracle.

$s p l$, spiracular lobe of pronotum.

$s p m b$, spiracular membrane.

$S p t D$, spermathecal duct.

$S t$, stipes.

$S t l$, stylet (united second valvulae).

Syr, salivary syringe.

$T$, tergum.

Tar, tarsus.

$T b$, tibia.

$T B$, tentorial bridge.

$T g$, tegula.

$T r$, trochanter.

Tra, trachea.

Tri, triangular plate of sting (first valvifer).

$U n$, unguis (lateral claw of pretarsus).

$U t r$, unguitractor plate.

$V$, venter.

$V D p h$, ventral diaphragm.

$V l v$, valve on lancet.

$W$, wing.

$W P$, pleural wing process.

\section{REFERENCES}

Arnhart, L.

1923. Das Krallenglied der Honigbiene. Archiv Bienenkunde, vol. 5, pp. 37-86, I pl.

Betts, Annie D.

1923. Practical bee anatomy, 88 pp., I2 pls. The Apis Club, Benson, England.

Bisнор, G. H.

1920. Fertilization in the honeybee. Journ. Exp. Zool., vol. 31, pp. 225-265, 3 pls.

Duncan, C. D.

1939. A contribution to the biology of North American vespine wasps. Stanford Univ. Publ., Biol. Sci., vol. 8, No. I, 272 pp., 54 pls. 
Ferris, G. F.

1940. The myth of the thoracic sclerites of insects. Microentomology, vol. 5 , pp. $87-90$.

Leuenterger, F.

I929. Les abeilles, anatomie et physiologie, I95 pp., I04 figs. Paris.

MORISON, G. D.

1927. The muscles of the adult honey-bee (Apis mellifera L.). Quart. Journ. Micr. Sci., vol. 7I, pp. 395-463, I2 figs.

PARK, W.

1925. The storing and ripening of honey by honeybees. Journ. Econ. Ent., vol. I8, pp. 405-410, 3 pls.

Rietschel, P.

1937. Bau und Funktion des Wehrstachels der staatenbildenden Bienen und Wespen. Zeitschr. Morph. Ökol. Tiere, vol. 33, pp. 313-357, 20 figs.

ShAFER, G. D.

1917. A study of the factors which govern mating in the honey bee. Michigan Agr. Coll. Div. Ent., Techn. Bull., No. 34, I9 pp., 3 pls.

SNODGRASS, R. E.

1925. Anatomy and physiology of the honey bee, 327 pp., I08 figs. New York.

I933. Morphology of the insect abdomen. Part II. The genital ducts and the ovipositor. Smithsonian Misc. Coll., vol. 89, No. 8, I48 pp., 48 figs.

I94I. The male genitalia of Hymenoptera. Smithsonian Misc. Coll., vol. 99, No. I4, 86 pp., 33 pls., 6 text figs.

WolfF, O. J. B.

I875. Das Riechorgan der Biene. Nova Acta. Ksl. Leop.-Carol. Deutschen Acad. Naturf., vol. 38, No. I, 25 I pp., 8 pls.

ZANDER, E.

I9II. Der Bau der Biene, I82 pp., 20 pls., I49 text figs. Stuttgart. 


\section{$2 \mathrm{BHL}$ Biodiversity Heritage Library}

Snodgrass, R. E. 1942. "The skeleto-muscular mechanisms of the honey bee." Smithsonian miscellaneous collections 103, 1-120.

View This Item Online: https://www.biodiversitylibrary.org/item/36094

Permalink: https://www.biodiversitylibrary.org/partpdf/32495

\section{Holding Institution}

Smithsonian Libraries

\section{Sponsored by}

Smithsonian

\section{Copyright \& Reuse}

Copyright Status: NOT_IN_COPYRIGHT

Rights: https://www.biodiversitylibrary.org/permissions/

This document was created from content at the Biodiversity Heritage Library, the world's largest open access digital library for biodiversity literature and archives. Visit BHL at https://www.biodiversitylibrary.org. 